\title{
International Society for Disease Surveillance Conference 2010
}

\section{Track 2: Public Health Surveillance}

4. The use of patient temperature data for biosurveillance in the emergency department DG Cochrane and JR Allegra

6. Maryland ESSENCE expansion to incorporate prescription medication data S Aslam, I Ajit, S Adams, and Z Faigen

7. Utilities of Maryland's syndromic surveillance system: indentifying threats, case investigation and situational awareness S Aslam, I Ajit, S Adams, and Z Faigen

9. Syndrome evaluation of individuals reporting emergency department utilization for notifiable disease and conditions T Azarian, A Kite-Powell, and S Zaheer

11. Tracking emergency department (ED) patients with gastrointestinal symptoms during a Norovirus epidemic in Miami-Dade R Borroto, J Suarez, E Rico, E O'Connell, A Llau, L Londono, P Mann, and G Zhang

13. A collaboration to enhance detection of disease outbreaks clustered by time of patient arrival H Burkom, L Ramac-Thomas, R Arvizu, C Lee, W Loschen, R Wojcik, and A Kite-Powell

14. Influenza mortality surveillance using a statewide electronic death registration system F Nebraska, 2009-2010 BF Buss, R Williams, P Pillai, and T Safranek

16. Population-based surveillance for methicillin-resistant Staphylococcus aureus infections among high school football and wrestling participants-Nebraska, 2008-2010 BF Buss and TJ Safranek

18. Influenza surveillance using inpatient health information exchange data N Close, PF Bugni, and K Lofy

20. Biosurveillance applications for resource-limited settings: open ESSENCE and ESSENCE desktop edition JS Coberly, RA Wojcik, JF Skora, AM Poku, ZC Whitley, TC Campbell, CJ Hodanics, SL Lewis, and DL Blazes

22. Relationship between neighborhood poverty and emergency department utilization for fever/flu syndrome K Desy, K Purviance, M Cartter, and JL Hadler

24. A comparison of the fever-flu syndrome category with the SC ILINet surveillance system in South Carolina: 2009-2010 influenza season H Dhotre, C Springs, D Drociuk, and YE Cheng

26. Can all-cause school absenteeism be used to optimize the timing of school closure in response to pandemic influenza? JR Egger, AG Hoen, E Wilson, JS Brownstein, DL Buckeridge, and KJ Konty

28. Evaluating the utility of school absenteeism during the 2009-2010 influenza season in Los Angeles County C Faustino, P Araki, E Kajita, M Jones, and B Hwang

30. Comparison of the US outpatient influenza-like illness surveillance network and the Google Flu Trends from 2008 to 2010 A Fowlkes, M Mohebbi, S Cook, C Conrad, L Brammer, and L Finelli

32. Using influenza rapid test positivity as an early indicator for the onset of seasonal influenza LH Gren, SC Alder, EA Joy, JL Lyon, CA Porucznik, and CJ Staes

34. Creating a shared epidemiologic vocabulary: lessons from the former Soviet Union A Grigoryan, C Clarke, T Chumachenko, and B Smoak

36. Effect of expanded recommendations for pediatric seasonal influenza vaccination: an international comparison AG Hoen, DL Buckeridge, KML Charland, KD Mandl, and JS Brownstein

38. Role of continuous monitoring on performance of surveillance systems in military populations in resource-limited settings RA Hora, DM Vera, ZJ Silvera, and JM Montgomery

40. BioSense program redesign TA Kass-Hout, BL Massoudi, L Rojas-Smith, SC Kaydos-Daniels, JS Brownstein, DL Buckeridge, and J Buehler 
42. The utility of inpatient data obtained from regional health information organizations for pneumonia and influenza surveillance G Johnson, X Jin, J-H Chen, H-G Chang, and D Morse

44. Florida's ESSENCE systemFfrom syndromic surveillance to routine epidemiologic analysis across syndromic and non-syndromic data sources A Kite-Powell, J Hamilton, R Wojcik, W Loschen, and R Hopkins

46. Comparison of Distribute and ILINet for national influenza surveillance K Kniss, S Benoit, A McIntyre, D Buckeridge, J Brownstein, L Brammer, and L Finelli

48. Emergency department diagnosis code data for surveillance of vaccine adverse events: comparison with the national vaccine adverse event reporting system E Lamb, H Vaughan-Batten, NJM Dailey, J-M Maillard, L Johns, AT Fleischauer, Z Moore, and M Davies

50. Meaningful use and public health surveillance: to travel fast or far? LA Lenert

52. Surveillance for nationally notifiable infectious conditions using ICD-9-CM diagnosis codes in the VA ESSENCE biosurveillance system

C Lucero, P Schirmer, G Oda, and M Holodniy

54. Using laboratory and medical encounter records to identify impact of prophylaxis policies on group A Streptococcus in three recruit training facilities TM Luse, K Shannon, and G Nowak

56. EpiSentry ${ }^{\mathrm{TM}}$ : simulation-based threat identification, response management and decision support for outbreaks and pandemics DW Mazeroski and T Arthur-Hartranft

58. Quantifying the relationship between influenza-related emergency department visits and hospital admissions in BioSense AF McIntyre, L Finelli, HS Burkom, SR Benoit, KL Kniss, and TA Kass-Hout

60. Evaluation of the NATO Disease Surveillance System in Kosovo in 2010 J-B Meynard, H Chaudet, L Pellegrin, Y Moreno, O Deboosere, P Mornand, K Pennin, S Stein, B Queyriaux, H-U Holterm, A Zuendorf, J Kocik, R Migliani, X Deparis, and G Texier

62. Creating a fast and flexible syndromic surveillance reporting system J Morin, A Ali, D Ferguson, RG Gutierrez, A Riley, N Vinson, S McClinton, C McDermaid, K Ali, J Martin, and RF Davies

64. Comparison of respiratory, febrile and influenza-like illness syndromes to detect laboratory-reported H1N1 and RSV, Influenza Season 2009-10, New York City

E Westheimer, TQ Nguyen, M Paladini, D Weiss, and S Balter

66. Early Aberration Reporting System (EARS) update: present and future G Rainisch, and K Hayashi

68. Description of the quality of public health case reports received at a local health department and potential impact on workflow D Rajeev, A Price, K Schnirel, M Hill, C Staes, and I Risk

70. Asthma patterns in Boston emergency department visits for children age five and under M Rennick, J Gunn, M Donovan, and J Salvia

72. Evaluating the use of syndromic surveillance for the detection of influenza-like illness in Salt Lake County, Utah J Robertson and M Hill

74. School absenteeism and emergency department ILI rates in King County, WA 2003-2009

C Rodriguez, A Baer, J Baseman, I Painter, D Yanez, T Koepsell, and JS Duchin

76. Impact of alternate diagnoses on the accuracy of influenza-like illness case definition used for H1N1 screening in the emergency department

D Rumoro, J Silva, S Shah, J Bayram, M Hallock, G Gibbs, M Waddell, and K Thomas

78. Access to and use of syndromic surveillance information at the local health department level E Samoff, A Ising, A Waller, E Sickbert-Bennett, M Davis, M Park, SW Haas, L DiBiase, and PDM MacDonald

80. Tracking H1N1 vaccine doses administered using CRA system B Nichols, S Waite, H Hill, L Han-Lee, S Sapkota, and J Tropper

81. User requirements for a user-centered design (UCD) redesign of a public health surveillance system: BioSense REN Sembajwe, TR Farris, OL Rojas-Smith, BL Massoudi, AK Banger, and A Casoglos

83. Using Twitter to estimate H1N1 influenza activity A Signorini, PM Polgreen, and AM Segre

84. How did specific specialties and new sentinel providers affect ILINet data post 2009 A/H1N1 recognition O Simwale

85. Use of a public health working group to coordinate multi-jurisdiction response to bioterrorism surveillance signals and influenza outbreaks AJ Siniscalchi

87. A comparison of syndromic surveillance chief complaint data and discharge data in a pediatric hospital system during 2009 H1N1 D Peace, W Smith, A Reeves, D Little, K Soetebier, and C Drenzek 
89. Design and development of a standards-based model to publish public health reporting criteria C Staes, D Rajeev, R Bradshaw, S Mottice, Z Lui, N Hulse, and S Narus

91. Use of emergency department data for case finding following a community anthrax exposure D Swenson, LK Mayo, K Dufault, ER Daly, S Bascom, J Dionne-Odom, and EA Talbot

93. Prospective space-time analysis of the 2007 cryptosporidiosis outbreak, Salt Lake County, Utah M Taddie, and I Yamada

95. An automated influenza-like-illness reporting system using freetext emergency department reports F-C Tsui, H Su, J Dowling, RE Voorhees, JU Espino, and M Wagner

97. The United States Department of Veterans Affairs Integrated Operations Center (VA IOC): collaborations for surveillance, analysis, and prediction for infectious disease threat preparedness-pilot review of dengue occurrence KS Wallace, GA Roselle, S Gamage, D Green, LA Simbartl, M Villeneuve, J Lacey, SM Kralovic, and JD Riojas

99. Syndromic surveillance of influenza-like illness using automated VA data preliminary results from the Idaho infectious disease reporting network WG Weppner, AP Wilper, J Mitten, J Tiumalu, R Tivis, and D Stevens

101. Evaluating University syndromic surveillance systems during the $2009 \mathrm{H} 1 \mathrm{~N} 1 \mathrm{influenza}$ pandemic Y Zhang, L May, and MA Stoto 


\title{
The use of patient temperature data for biosurveillance in the emergency department
}

\author{
DG Cochrane, and JR Allegra
}

New Jersey Research Foundation, Morristown Memorial Hospital Residency in Emergency Medicine, Morristown, NJ, USA

E-mail: johnalle@verizon.net

\section{Introduction}

Biosurveillance systems commonly use emergency department (ED) patient chief complaint data (CC) for surveillance of influenza-like illness (ILI). Daily volumes are tracked using a computerized patient CC classifier for fever (CC Fever) to identify febrile patients. Limitations in this method have led to efforts to identify other sources of ED data. At many EDs the triage nurse measures the patient's temperature on arrival and records it in the electronic medical record (EMR). This makes it possible to directly identify patients who meet the CDC temperature criteria for ILI: temperature greater than 100 degrees $\mathrm{F}(\mathrm{T}>100 \mathrm{~F})$.

\section{Objective}

To evaluate whether a classifier based on temperature $>100 \mathrm{~F}$ would perform similarly to CC Fever and might identify additional patients.

\section{Methods}

Design: Retrospective review of EMR data. Setting: Five EDs in New York and New Jersey from 10-1-07 to 5-31-09. Protocol: For patients with a measured temperature, we determined daily volumes for CC Fever and for T>100 and compared them using linear regression analysis and visual inspection of the time-series graphs. We also determined the additional

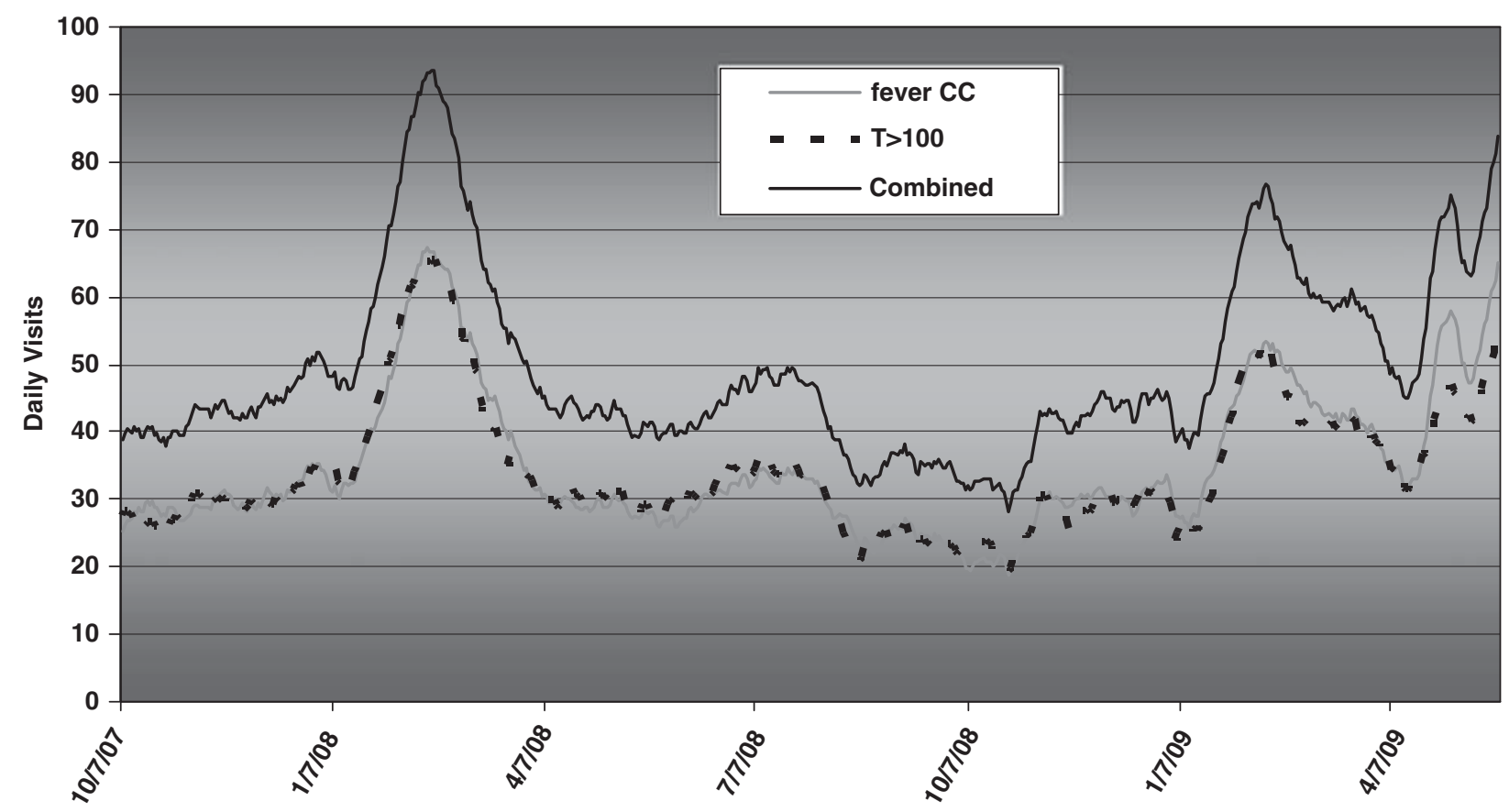

Figure 1 14-day moving average of visits for fever $C C, T>100$ and combined fever $C C$ and $T>100$ classifiers.

This is an Open Access article distributed under the terms of the Creative Commons Attribution licence (http://creativecommons.org/licenses/by/2.5) OPEN - ACCESS which permits unrestricted use, distribution, and reproduction in any medium, provided the original work is properly cited. 
number of visits that were identified by adding $\mathrm{T}>100$ to CC Fever.

\section{Results}

There were a total of 352742 ED visits, of which 265099 (75\%) had a measured temperature. Within that group, the total volumes were 21411 for CC fever and 20876 for $\mathrm{T}>100$. The correlation coefficient for the daily volumes was $r^{2}=0.94, P<0.001$. Visual inspection of the time-series graphs (see Figure 1) showed a close match of seasonal peaks. The $\mathrm{T}>100$ classifier identified an additional 8959 patients not identified by the CC fever classifier alone (42\% increase).

\section{Conclusions}

The classifier for measured temperature identified seasonal fever peaks similarly to the classifier based on CC fever and identified many additional patients. Further study is needed to determine the potential advantage of using measured temperature in ED surveillance for ILI.

\section{Acknowledgements}

This paper was presented as a poster at the 2010 International Society for Disease Surveillance Conference, held in Park City, UT, USA on 1-2 December 2010. 


\title{
Maryland ESSENCE expansion to incorporate prescription medication data
}

\author{
S Aslam, I Ajit, S Adams, and Z Faigen \\ Office of Preparedness and Response, Maryland Department of Health and Mental Hygiene, Baltimore, MD, USA \\ E-mail: saslam@dhmh.state.md.us
}

\section{Objective}

The purpose of this paper is to describe Maryland's process of enhancing its Electronic Surveillance System for the Early Notification of Community-based Epidemics (ESSENCE) by incorporating additional data sources such as prescription medication data.

\section{Introduction}

Maryland has a powerful syndromic surveillance system, ESSENCE, which is used for the early detection of disease outbreaks, suspicious patterns of illness, and public health emergencies. ESSENCE incorporates traditional and nontraditional health indicators from multiple data sources (emergency department chief complaints, over-the-counter (OTC) medication sales, and poison control center data).

Initially, $15(30 \%)$ acute care hospitals in the National Capital Region and Baltimore Metro Region of the state were sending emergency department (ED) data to ESSENCE. DHMH began planning several years ago to increase the number of hospitals reporting to ESSENCE.

In 2007, Maryland's Governor introduced a homeland security initiative that outlined 12 Core Goals for A Prepared Maryland. One of core goals was to improve biosurveillance and in 2009, Maryland successfully incorporated 100\% (45) acute-care hospitals into ESSENCE. Maryland continues to enhance and improve ESSENCE by incorporating additional data sources such as prescription medication data.

\section{Methods}

First, Maryland contacted the agency which developed ESSENCE, to learn about what data and software developments needs to occur in order to add another data source into ESSENCE system. Next, Maryland started on efforts to obtain the prescription medication data.

Currently, there is only one state that collects prescription medication data for biosurveillance. Maryland contacted the state to learn more about how this process is setup in their state.

With the help of Board of Pharmacy, Maryland started to work with chain pharmacies that were already sending OTC data to ESSENCE to incorporate prescription medication and
Table 1 List of surveillance medications

Amino-penicillins

Tetra-cyclines

Quinolones/Fluoroquinolones

Neuraminidase Inhibitors

M2 Inhibitors

an additional pharmacy that was participating in the State's Antiviral purchase program. Although pursing efforts to obtain data from pharmacies, Maryland learned that Center for Disease Control and Prevention (CDC) already receives prescription medication data from a third party vendor. Maryland contacted CDC and learned that CDC has data, which Maryland is seeking and is willing to share it with State.

\section{Results}

Currently, Maryland is the in the process of working with its partners to incorporate the prescription medication data into ESSENCE. Maryland will have a fully automated system that creates alerts and allows near real-time surveillance on antivirals and prescription medications used to treat category A agents (see Table 1 above).

\section{Conclusion}

Maryland will be one of the first States to incorporate prescription medication data into its syndromic surveillance system. Addition of prescription medication into ESSENCE will make Maryland's syndromic surveillance more robust and enhance its capability for early detection of outbreaks and public health emergencies. ESSENCE will continue to be a tool used for situational awareness and inform decision makers in Maryland. Lastly, the process of incorporation prescription medication has provided an opportunity for Maryland to collaborate with other partners and CDC.

\section{Acknowledgements}

This paper was presented as a poster at the 2010 International Society for Disease Surveillance Conference, held in Park City, UT, USA on 1-2 December 2010.

open 2 Access This is an Open Access article distributed under the terms of the Creative Commons Attribution licence (http://creativecommons.org/licenses/by/2.5) OPEN - ACCESS which permits unrestricted use, distribution, and reproduction in any medium, provided the original work is properly cited. 


\title{
Utilities of Maryland's syndromic surveillance system: indentifying threats, case investigation and situational awareness
}

\author{
S Aslam, I Ajit, S Adams, and Z Faigen \\ Office of Preparedness and Response, Maryland Department of Health and Mental Hygiene, Baltimore, MD, USA \\ E-mail: saslam@dhmh.state.md.us
}

\section{Objective}

The purpose of this paper is to describe how Maryland's syndromic surveillance system, electronic surveillance system for the early notification of community-based epidemics (ESSENCE), has many utilities including identifying threats, case investigation and situational awareness.

\section{Introduction}

Maryland's electronic surveillance system for the early notification of community-based epidemics (ESSENCE) data includes emergency department visits from all acute care hospitals, over-the-counter medication sales and poison control data that cover all jurisdictions in Maryland.

Maryland Department of Health and Mental Hygiene (DHMH) uses ESSENCE daily for the early detection of public health emergencies. DHMH also utilizes ESSENCE for other purposes including situational awareness during high security events, assistance with outbreak investigation and for the $\mathrm{H} 1 \mathrm{~N} 1$ pandemic.

\section{Methods}

ESSENCE data are reviewed closely on a daily basis looking for clusters indicating a public health threat. However, during the 2008 nationwide Salmonella outbreak, a query was developed to search for patients with chief complaints related to Salmonella or tomatoes from April to June 2008.

During the initial H1N1 outbreak, DHMH used ESSENCE as a tool to enhance case finding efforts. In addition, throughout the H1N1 response, ESSENCE was also used to monitor levels of ILI across the state. Daily reports were provided to the Governor's office and state executives to enhance situational awareness and inform decision-making.

In 2009, ESSENCE was used for situational awareness during the Presidential inauguration. The following list of queries was used during the inaugural period: symptoms for hypothermia, injury, dehydration, influenza like illness, meningitis and Center of Disease Control and prevention category A diseases (anthrax, botulism, plague, small pox, tularemia and viral hemorrhagic fever).

Most recently in 2010, ESSENCE was queried to identify the number of patients that reported to Maryland hospitals for prophylaxis after being exposed to a rabid calf.

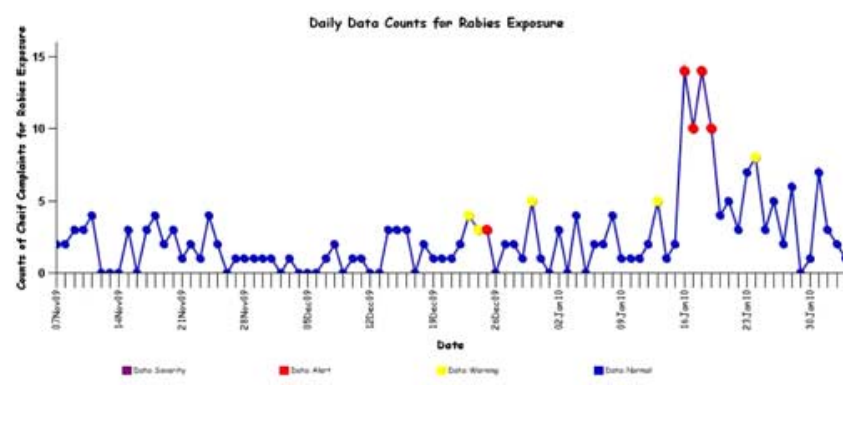

\begin{abstract}
Results
On numerous occasions during the daily review, ESSENCE has identified clusters of meningitis. A statistically significant increase in ILI activity was observed during the H1N1 pandemic in spring and fall of 2009. Monitoring of the overthe-counter medication sales allows for the early prediction of an increase in ILI activity. During the 2009 Presidential inauguration, ESSENCE identified a statistically significant increase in the number of hypothermia cases. During the 2008 Salmonella outbreak, ESSENCE identified a case. This case was detected in ESSENCE 1 week earlier than its report date to the state health department and about 3 weeks earlier than when it was identified as part of the outbreak. Lastly, ESSENCE was utilized to monitor the number of cases that reported to Maryland hospitals for prophylaxis after being exposed to a rabid calf.
\end{abstract} This is an Open Access article distributed under the terms of the Creative Commons Attribution licence (http://creativecommons.org/licenses/by/2.5)
which permits unrestricted use, distribution, and reproduction in any medium, provided the original work is properly cited. 


\section{Conclusion}

ESSENCE is a vital tool for DHMH that provides a timely and ongoing situational awareness for policy decision makers. ESSENCE is a useful tool during public health events such as outbreaks and pandemic.

\section{Acknowledgements}

This paper was presented as a poster at the 2010 International Society for Disease Surveillance Conference, held in Park City, UT, USA on 1-2 December 2010. 


\title{
Syndrome evaluation of individuals reporting emergency department utilization for notifiable disease and conditions
}

\author{
T Azarian ${ }^{1}$, A Kite-Powell ${ }^{2}$, and S Zaheer ${ }^{1}$ \\ ${ }^{1}$ Duval County Health Department, Jacksonville, FL, USA; and ${ }^{2}$ Bureau of Epidemiology, Tallahassee, FL, USA \\ E-mail:Taj_Azarian@doh.state.fl.us
}

\section{Objective}

This paper characterizes emergency department (ED) utilization among individuals diagnosed and reported with notifiable diseases and conditions (NDC). Furthermore, it evaluates the subsequent assignment of syndromic surveillance (SS) syndromes based on the patient's chief complaint (CC) during their ED visit.

\section{Introduction}

The Duval County Health Department (DCHD) serves a community of over one million people in Jacksonville, FL, USA. Each year, DCHD Epidemiology Program reports an average of 1133 (4-year average) NDC with the exception of STD/HIV, TB, and viral hepatitis. Within Duval County, emergency medical care is provided by eight local hospitals, including one pediatric facility and a level-1 trauma center. These facilities contribute SS CC data to the Electronic Surveillance System for Early Notification of Communitybased Epidemics (ESSENCE) of Florida.

Historically, evaluations of SS systems have used ICD-9 diagnoses as the gold standard to determine predictive values. ${ }^{1}$ However, limited studies have surveyed reports of NDC to identify related ED visits and subsequent CC-based syndrome categorization. These data may provide public health investigators insight into health seeking behaviors, interpretation of SS signals, and prevalence of NDC within ED data.

\section{Methods}

Between March and August 2010, DCHD epidemiology investigators collected ED visitation history from reported cases of NDC. ED usage during their course of illness was documented. An ESSENCE review was then conducted to match cases reporting ED visitation. To reduce the burden on hospital staff, cases were matched by ED, date of visit, age, zip code, sex, and race, instead of ESSENCE unique patient identifier. Case investigation data was then abstracted from
Merlin, NDC reporting registry of Florida. ESSENCE syndromes were compared with appropriate NDC-specific syndromes determined by CDC ICD-9-based syndrome definitions. ${ }^{2}$ Prevalence of notifiable diseases within ED SS data was evaluated. Comparisons between populations using ED services and those not reporting ED visitation were made.

\section{Results}

From 11 March 2010 to 20 August 2010, 486 NDC were reported by DCHD. In all, 61 cases were not directly interviewed. Of the remaining 425, 53.2\% (226) reported visiting an ED during the course of their illness. Of these, 198 $(87.6 \%)$ were diagnosed through the ED or during admission. The remaining 28 were diagnosed during subsequent visits to clinics or primary care providers. In all, 200 cases were able to be matched to the corresponding ED visits in ESSENCE. In addition, 13 cases reported two ED visits and 1 reported three visits for their illnesses; comprising a total of 215 visits associated with NDC.

Regarding syndrome classification, 58.14\% (125/215) of syndromes were correctly matched. In addition, 14.8\% (32/ 215) of visits were assigned to the 'other' category and not identified appropriately as a syndrome based on the reported NDC. The rash syndrome category had the highest proportion of matches. The gastrointestinal illness (GI) category had the highest frequency of related ED visits. The GI category also had the highest proportion of CC misclassified as a single syndrome; 30/154 (19.5\%) classified in the fever syndrome. This was most prominent among children $<2$ years old.

\section{Conclusions}

This study provides an evaluation of syndrome classification among individuals with reported NDC. In addition, this review revealed limitations for the use of SS data to provide trends for NDC. For instance, children $<2$ years old are

open Access This is an Open Access article distributed under the terms of the Creative Commons Attribution licence (http://creativecommons.org/licenses/by/2.5) OPEN - ACCESS which permits unrestricted use, distribution, and reproduction in any medium, provided the original work is properly cited. 
non-verbal and as a result, signs such as fever and rash can predominate CC of the caregiver. This was demonstrated by the misclassification of reported enteric illnesses within the fever syndrome among children $<2$ years old. Individuals may also present to EDs for unrelated illnesses and later be diagnosed with a NDC. Also, the seasonality of enteric NDC may have resulted in higher frequencies of GI visits during the study period. Overall, collection and evaluation of ED visitation histories during NDC case interviews has improved the local interpretation of SS signals and provided context for syndromic and NDC surveillance.

\section{Acknowledgements}

This paper was presented as an oral presentation at the 2010 International Society for Disease Surveillance Conference, held in Park City, UT, USA on 1-2 December 2010.

\section{References}

1 Lombardo J, Burkom H, Pavlin J. Centers for Disease Control and Prevention (CDC). II ESSENCE and the framework for evaluating syndromic surveillance systems. MMWR Morb Mortal Wkly Rep 2004;53 (Suppl): 159-65.

2 DHHS and CDC, syndrome definitions for diseases associated with critical bioterrorism-associated agents, 2003, http://www.bt.cdc. gov/surveillance/syndromedef/. 


\title{
Tracking emergency department (ED) patients with gastrointestinal symptoms during a Norovirus epidemic in Miami-Dade
}

\author{
R Borroto, J Suarez, E Rico, E O'Connell, A Llau, L Londono, P Mann, and G Zhang \\ Office of Epidemiology, Disease Control and Immunization Service (EDC-IS), Miami-Dade County Health Department (MDCHD), Miami, FL, USA \\ E-mail: Rene_Borroto-Ponce@doh.state.fl.us
}

\section{Objective}

To demonstrate how the Epidemiology, Disease Control and Immunization Service (EDC-IS) at the Miami-Dade County Health Department used ESSENCE to track gastrointestinal symptoms during a Norovirus epidemic.

\section{Introduction}

From January to March 2010, thirteen outbreaks of Norovirus infection were reported to the EDC-IS, up from four outbreaks in the entire 2008 and same number during 2009. Individual cases of Norovirus are not reportable in the State of Florida. That makes it difficult to track the onset, rise, peak, and fading of epidemics of this disease.

\section{Methods}

A countywide query with the string nausea, or vomit, or diarrhea, or gastroenteritis is performed in ESSENCE on a daily basis. The incidence of enteric illnesses other than Norovirus was analyzed by comparing the count of the period January-March 2010 to the mean of the same months from 2000 through 2009. The reportable diseases included in the analysis were of bacterial origin (shigellosis, Escherichia coli infection, campylobacteriosis, and salmonellosis) and parasitic nature (cyclosporiasis, cryptosporidiosis, and giardiasis), as notified in the Merlin database of reportable diseases of the State of Florida. No outbreak was notified during the January-March 2010 period among non-reportable enteric diseases (that is, caused by Bacillus cereus, Clostridium perfringens, Plesiomonas shigelloides, Aeromonas, and Staphylococcus). As for non-reportable rotavirus infection, the monthly count of diagnosed children aged 0-4 years from January 2003 to April 2010 at the largest pediatric hospital in Miami-Dade was analyzed.

\section{Results}

There had been a spike of emergency department (ED) patients right after Christmas in late December 2009 (see Figure 1), but, unlike the spikes of January-March 2010, it

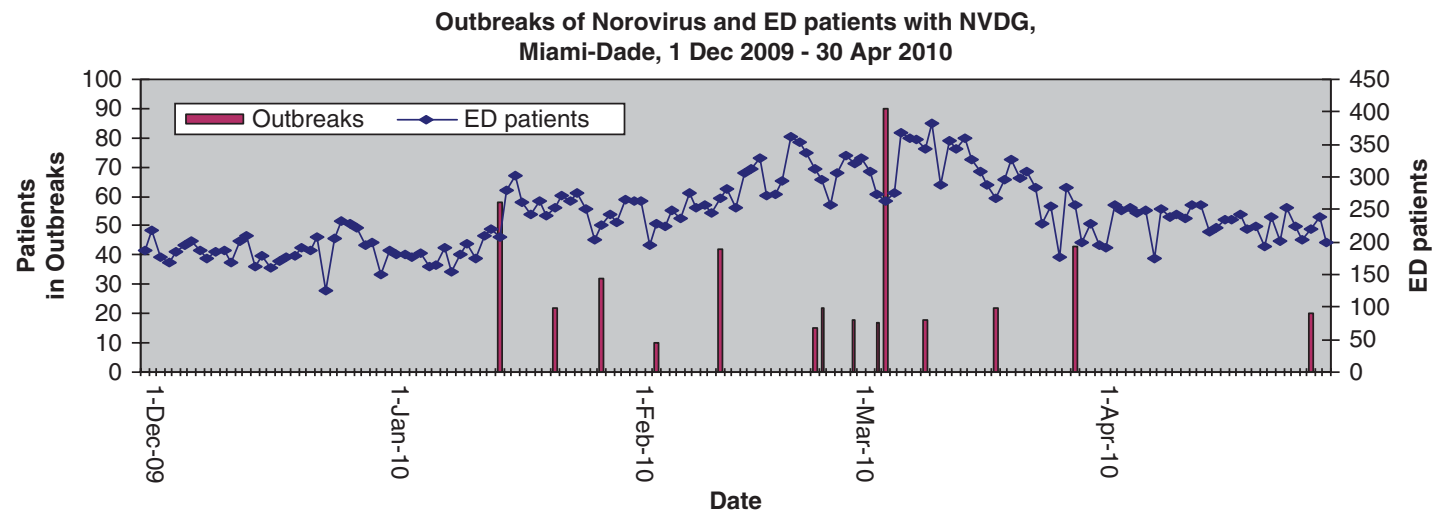

Figure 1 Norovirus outbreaks and ED patients with gastrointestinal symptoms.

This is an Open Access article distributed under the terms of the Creative Commons Attribution licence (http://creativecommons.org/licenses/by/2.5) OPEN ACCESS which permits unrestricted use, distribution, and reproduction in any medium, provided the original work is properly cited. 
occurred only among adults, and spared children. This spurt in December returned to background levels after 3 days, unlike the spikes of January-March 2010. Moreover, no outbreak of Norovirus was reported in December 2009. The first outbreak of 2010 had its onset on Wednesday, 13 January, whereas the first significant increase in ED patients occurred from 16 (Saturday) to 18 (Monday) January, during the Dr Martin Luther King Jr holiday weekend, when 3 consecutive days of red flags were posted among the all-age group. The next leg up in the uptrend of the count of ED patients came right after St Valentine Day, when four consecutive flags were posted from 15 through 18 February. The count of ED patients peaked in early March 2010, when the number of Norovirus outbreaks also peaked. The only reportable enteric disease that showed a peak in March 2010 was campylobacteriosis, mostly because of changes in the case definition, rather than an actual increase in incidence. Rotavirus infection did not appear to make a contribution to the increase in the count of ED patients.

\section{Conclusions}

ESSENCE can help tracking epidemics of Norovirus infection, although a careful analysis of the temporal patterns of the incidence of other enteric illnesses should be ascertained.

\section{Acknowledgements}

This paper was presented as a poster at the 2010 International Society for Disease Surveillance Conference, held in Park City, UT, USA, on 1-2 December 2010. 


\title{
A collaboration to enhance detection of disease outbreaks clustered by time of patient arrival
}

\author{
H Burkom ${ }^{1}$, L Ramac-Thomas ${ }^{1}$, R Arvizu $^{1}$, C Lee $^{1}$, W Loschen ${ }^{1}$, R Wojcik ${ }^{1}$, and A Kite-Powell ${ }^{2}$ \\ ${ }^{1}$ Johns Hopkins University Applied Physics Laboratory, National Security Technology Department, Baltimore, MA, USA; and ${ }^{2}$ Florida \\ Department of Health, Bureau of Epidemiology, Tallahassee, FL, USA \\ E-mail: howard.burkom@jhuapl.edu
}

\section{Objective}

This presentation discusses the approach and results of collaboration to enable a solution of a hospital time-of-arrival (TOA) monitoring problem in syndromic surveillance applied to public health data at the hospital level for county monitoring.

\section{Introduction}

One objective of public health surveillance is detecting disease outbreaks by looking for changes in the disease occurrence, so that control measures can be implemented and the spread of disease minimized. For this purpose, the Florida Department of Health (FDOH) employs the Electronic Surveillance System for Early Notification of Community-based Epidemics (ESSENCE). The current problem was spawned by a laborintensive process at the FDOH: authentic outbreaks were detected by epidemiologists inspecting ESSENCE time series and derived event lists. The corresponding records indicated that patients arrived at an ED within a short interval, often less than 30 minutes. TOA task was to develop and automate a capability to detect events with clustered patient arrival times at the hospital level for a list of subsyndrome categories of concern to the monitoring counties.

\section{Methods}

Factors that drove the choice of the statistical alerting algorithm for the TOA surveillance were: user objectives and processing performance needs, the existing ESSENCE operational framework, and the limitation to time series and covariates that could be extracted quickly with ESSENCE queries.

The chosen approach was to monitor each of the many time-series cells, in which cells could be stratified by several variables. Following analysis of the influence of selected variables in historical data, a cell was defined as a hospital/ syndrome/time-of-day combination (Figure 1). For the majority of these cells, a Poisson distribution test was applied, and a negative binomial test was applied for cells whose time-series counts were overdispersed. The method adjusts for daily patterns and for levels of activity that vary widely across hospitals.

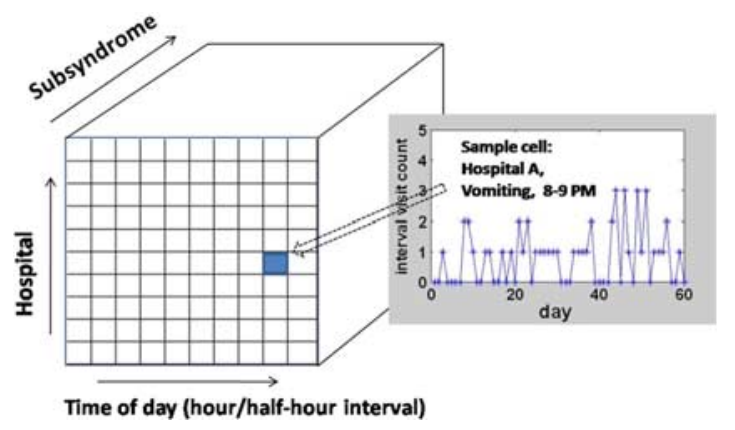

Figure 1 Schematic depicting the temporal cell-based monitoring method.

\section{Results}

This method was tested and validated on ED visit count data covering over 6 years and aggregated at 30-minute intervals for 51 subsyndromes from each of 134 hospitals covering 34 Florida counties.

An alerting threshold of $P=0.0001$ gave detection of 18 of the 20 known signals in the data, with manageable alert counts for the most common subsyndromes in the largest hospitals. This method is implemented in an operational ESSENCE website module.

\section{Conclusion}

The TOA application resulted from (a) a well-defined problem and associated constraints; (b) involvement of all stakeholders for the duration of the project, including a state-level epidemiologist domain expert; and (c) data streams sufficient for a useful solution. The approach and implementation were the result of a give-and-take process informed by data analysis, with features that were informative for the efficiency of future collaborative efforts.

\section{Acknowledgements}

This paper was presented as an oral presentation at the 2010, International Society for Disease Surveillance Conference, held in Park City, UT, USA on 1-2 December 2010.

open -2 access This is an Open Access article distributed under the terms of the Creative Commons Attribution licence (http://creativecommons.org/licenses/by/2.5) OPEN - ACCESS which permits unrestricted use, distribution, and reproduction in any medium, provided the original work is properly cited. 


\title{
Influenza mortality surveillance using a statewide electronic death registration system - Nebraska, 2009-2010
}

\author{
BF Buss $^{1,2}$, R Williams ${ }^{1}$, P Pillai ${ }^{1,2}$, and T Safranek ${ }^{1}$ \\ ${ }^{1}$ Nebraska Department of Health and Human Service, Lincoln NE, USA; and ${ }^{2}$ Centers for Disease Control and Prevention, CDC Office of Public \\ Health Preparedness, Atlanta, GA, USA \\ E-mail: bryan.buss@nebraska.gov
}

\section{Objective}

This report describes use and evaluation of a near real time, novel electronic influenza mortality surveillance system developed in Nebraska.

\section{Introduction}

Public health surveillance using death data is critical for tracking the impact of diseases such as influenza. However, utility of such systems is compromised by delayed reporting, particularly when it is paper based. In Nebraska, funeral directors are encouraged to initiate death certificates electronically by an electronic death registration system (EDRS). Although paper-based or mixed (electronic followed by paper) registration is still accepted statewide, EDRS usage has gradually increased over time. Fact of death (FOD) data that includes time and place of death, and a deceased person's identity are usually recorded by a funeral director. Cause of death data in the medical portion are provided by physicians or medical examiners at a later date. FOD data entered into EDRS are immediately available, whereas paper-based data must first be mailed to vital records whereupon staff enter it into EDRS. Although implemented in 2006, epidemiology surveillance staff did not have realtime access to EDRS data until early 2009, when a collaboration was formed between the Office of vital records and the Office of epidemiology within the Nebraska Department of Health and Human Services. Daily electronic access by surveillance staff to death certificate data was established enabling the conductance of public health death surveillance.

\section{Methods}

Timeliness of electronically-filed versus paper-based FOD data was evaluated from 19 July 2009 to 1 May 2010. Additionally, sensitivity and specificity of an influenzaspecific death surveillance system using EDRS were com- pared with active surveillance using laboratory and provider reports also conducted from 19 July to 1 May 2010. For active surveillance, an influenza-associated death was defined as a death, with laboratory confirmation or clinical impression of influenza upon chart review. For surveillance by EDRS, funeral directors were instructed during this time period to query decedent's next-of-kin whether the death 'was in any way associated with influenza.' Responses including yes, no, or unknown were entered into a new field, created specifically in the FOD section in EDRS for purposes of influenza death surveillance. Deaths reportedly associated with influenza were investigated to ascertain cases.

\section{Results}

Of 11,598 deaths, filed in Nebraska during 19 July 2009 to 1 May 2010, registration for a total of 7,354 (63.4\%) were initiated electronically by EDRS, for which FOD notice and data were available at a median of 10 days sooner than paper-based data (Table 1). Among the deaths registered by EDRS, $31(0.4 \%)$ were reportedly influenza associated. The remaining 7,323 (99.6\%) were classified as 'no,' 'unknown,' or had missing data. Active surveillance identified 21 influenza-associated deaths that met the case definition. Of these, 16 (76.2\%) were initiated electronically, and were included in the analysis; approximately half being reported by funeral directors as influenza associated

Table 1 Time interval in days from date of death to date when death registration was initiated

\begin{tabular}{lcccc}
\hline Initiation method & $\mathrm{N}$ & $\begin{array}{c}\text { Mean } \\
\text { (days) }\end{array}$ & $\begin{array}{c}\text { Median } \\
\text { (days) }\end{array}$ & $\begin{array}{r}\text { Range } \\
\text { (days) }\end{array}$ \\
\hline Electronic & 7354 & 2.0 & 1.0 & $0-113$ \\
Paper based & 4244 & 13.8 & 11.0 & $1-221$ \\
\hline
\end{tabular}

open Access This is an Open Access article distributed under the terms of the Creative Commons Attribution licence (http://creativecommons.org/licenses/by/2.5) OPEN - ACCESS which permits unrestricted use, distribution, and reproduction in any medium, provided the original work is properly cited. 
Table 2 Influenza-associated death notification, with EDRS, compared with active surveillance

\begin{tabular}{lrrr}
\hline Surveillance by EDRS & \multicolumn{2}{c}{ Active surveillance } & Total \\
\cline { 2 - 3 } & Case & Not a case & \\
\hline Case & 7 & 24 & 31 \\
Not a case & $9^{\mathrm{a}}$ & 7314 & 7323 \\
& 16 & 7338 & 7354 \\
\hline
\end{tabular}

Abbreviation: EDRS, electronic death registration system. 'aive 'no,' three 'unknown,' and one missing. Sensitivity $=43.8 \%$ (95\% confidence interval, 19.4-68.1). Specificity $=99.7 \%$ (95\% confidence interval, 99.5-99.8). Positive predictive value $=22.6 \%$ (95\% confidence interval, 7.9-37.3). Negative predictive value $=99.9 \%$ (95\% confidence interval, $99.8-100.0)$.

(sensitivity, 43.8\%). Overall, few false positives as a proportion of all deaths (specificity, 99.7\%) were reported (Table 2).

\section{Conclusion}

Nebraska's EDRS substantially enhances timeliness of FOD data availability. Although sensitivity and positive predictive values were limited, disease-specific death notification by funeral directors by EDRS holds promise as an early detection tool for emergent public health problems such as influenza-associated mortality. Additional training and refinement are needed to improve utility. Further study is needed to evaluate its utility during future influenza seasons when heightened public awareness attributable to 2009-2010 pandemic H1N1 has waned, and for mortality surveillance applied to other public health conditions.

\section{Acknowledgements}

This paper was presented as an oral presentation at the 2010, International Society for Disease Surveillance Conference, held in Park City, UT, USA on 1-2 December 2010 .

\section{Disclaimer}

The findings and conclusions in this report are those of the authors and do not necessarily represent the official position of the Centers for Disease Control and Prevention. Likewise, the authors' findings and conclusions do not necessarily represent the views of the Nebraska Department of Health and Human Services. 


\title{
Population-based surveillance for methicillin-resistant Staphylococcus aureus infections among high school football and wrestling participants - Nebraska, 2008-2010
}

\author{
BF Buss $^{1,2}$, and TJ Safranek ${ }^{1}$ \\ ${ }^{1}$ Nebraska Department of Health and Human Service, Lincoln, Nebraska, USA; and ${ }^{2}$ Centers for Disease Control and Prevention, Atlanta, \\ Georgia, USA \\ E-mail: bryan.buss@nebraska.gov
}

\section{Objectives}

The Nebraska Department of Heath and Human Services (NDHHS) Office of Epidemiology conducted ongoing surveillance to monitor statewide incidence of physiciandiagnosed methicillin-resistant Staphylococcus aureus (MRSA) infections among high school football and wrestling participants during school years 2008-09 and 2009-10.

\section{Introduction}

MRSA, which had been known primarily as a cause of healthcare-associated infections, increasingly has been recognized as a cause of skin infections among persons of all ages who have little or no contact with healthcare settings. Such infections are the most common cause of skin and soft-tissue infections among patients presenting to emergency departments. ${ }^{1}$ Community-associated MRSA infections among high school, college, and professional athletes, and outbreaks within teams have established otherwise healthy athletes as a population at risk. ${ }^{2-5}$ To monitor the occurrence of such infections among Nebraska high school athletes, the NDHHS Office of Epidemiology first initiated population-based surveillance to determine incidence during the 2006-07 and 2007-08 school years. Findings of this surveillance established MRSA as an emerging cause of infections among Nebraska high school athletes, particularly football and wrestling participants; from 2006-07 to 2007-08, physician-diagnosed MRSA incidence per 10,000 wrestlers increased from 19.6 to 60.1 , and incidence per 10,000 football players increased from 5.0 to $25.1 .^{6}$ In response to this finding, surveillance conducted at regular intervals has since been continued to monitor trends.

\section{Methods}

To facilitate ongoing surveillance, a list of official contacts in each of Nebraska's 312 high schools has been maintained and regularly updated. To foster collaboration and enhance response rates, MRSA prevention and control resources and surveillance findings are regularly provided to the targeted officials in each school.

During both the 2008-09 and 2009-10 school years, we developed four Internet-based questionnaires to survey delegated officials in all 312 Nebraska high schools, following the completion of the respective football and wrestling seasons. Each school's administration has delegated responsibility for completing the survey to an appropriate official associated with that school's athletic programs and having knowledge of procedures for prevention, identification, and control of infections among athletes. We collected number of participants for both football and wrestling in each school and number of athletes with physician-diagnosed MRSA infections to calculate statewide attack rates per 10,000 participants.

\section{Results}

Of 312 Nebraska schools, 239 (76.6\%) and 251 (80.4\%) responded to 2008-09 and 2009-10 football surveys, respectively. Of respondents, 97.5\% (233/239) and $98.4 \%$ $(247 / 251)$ had football programs of which $6.4 \%$ $(15 / 233)$ and $3.6 \%(9 / 247)$ reported $\geqslant 1$ physiciandiagnosed MRSA-affected participant. Similarly, of 312 schools, 177 (56.7\%), and 251 (80.4\%) responded to 200809 and 2009-10 wrestling surveys, respectively. Of respondents, $78.0 \%(138 / 177)$ and $75.3 \%(189 / 251)$ had wrestling programs of which $7.2 \%$ (10/138) and $7.4 \%$ $(14 / 189)$ reported $\geqslant 1$ physician-diagnosed MRSA-affected participant. Incidence per 10,000 football players decreased from 19.0 to 11.5 , and incidence per 10,000 wrestlers decreased from 60.8 to 50.0 from $2008-09$ to $2009-10$, respectively.

open Access This is an Open Access article distributed under the terms of the Creative Commons Attribution licence (http://creativecommons.org/licenses/by/2.5) OPEN - ACCESS which permits unrestricted use, distribution, and reproduction in any medium, provided the original work is properly cited. 


\section{Conclusions}

Estimated incidence of physician-diagnosed MRSA infections among Nebraska high school football and wrestling participants decreased substantially during 2009-10 compared with 2008-09, demonstrating that the apparent increasing incidence has not continued. By maintaining updated contact information of at least one official in each Nebraska high school and communicating regularly, we have continued surveillance with high rates of participation to monitor MRSA incidence among high school athletes over four consecutive school years.

\section{Acknowledgements}

This paper was presented as a poster at the 2010 International Society for Disease Surveillance Conference, held in Park City, UT, USA on 1-2 December 2010.

\section{References}

1 Moran GJ, Krishnadasan A, Gorwitz RJ, Fosheim GE, McDougal LK, Carey RB, et al. Methicillin-resistant S. aureus infections among patients in the emergency department. N Engl J Med 2006;355: 666-74.

2 Lindenmayer JM, Schoenfeld S, O'Grady R, Carney JK. Methicillinresistant Staphylococcus aureus in a high school wrestling team and the surrounding community. Arch Int Med 1998;158:895-9.

3 Romano R, Lu D, Holtom P. Outbreak of community-acquired methicillin-resistant Staphylococcus aureus skin infections among a collegiate football team. J Athl Train 2006;41(2): 141-5.

4 Nguyen DM, Mascola L, Brancoft E. Recurring methicillin-resistant Staphylococcus aureus infections in a football team. Emerg Infect Dis. 2005;11(4): 526-32.

5 Kazakova SV, Hageman JC, Matava M, Srinivasan A, Phelan L, Garfinkel B. A clone of methicillin-resistant Staphylococcus aureus among professional football players. N Eng J Med 2005;352:468-75.

6 Buss BF, Mueller SW, Theis M, Keyser A, Safranek TJ. Populationbased estimates of Methicillin-Resistant Staphylococcus Areus (MRSA) infections among high school athletes-Nebraska, 20062008. J Sch Nurs 2009;25(4): 282-91. 


\title{
Influenza surveillance using inpatient health information exchange data
}

\author{
N Close ${ }^{1}$, PF Bugni ${ }^{2}$, and K Lofy ${ }^{1}$ \\ ${ }^{1}$ Communicable Disease Epidemiology, Washington State Department of Health, Shoreline, WA, USA; and ${ }^{2}$ Center for Public Health \\ Informatics, University of Washington, Seattle, WA, USA \\ E-mail: natasha.close@doh.wa.gov
}

\section{Objective}

To evaluate the timeliness, completeness, and representativeness of influenza hospitalization data from an inpatient health information exchange.

\section{Introduction}

During the 2009 H1N1 influenza pandemic, the Washington State Department of Health (DOH) temporarily made labconfirmed influenza hospitalizations and deaths reportable. As reporting influenza hospitalizations is resource intensive for hospitals, electronic sources of inpatient influenza surveillance data are being explored. A large Health Information Exchange (WA-HIE) currently sends DOH the following data elements on patients admitted to 14 hospitals throughout eastern Washington: hospital, admission date, age, gender, patient zip code, chief complaint, final diagnoses, discharge disposition, and unique identifiers. WA-HIE inpatient data may be valuable for monitoring influenza activity, influenza morbidity, and the basic epidemiology of hospitalized influenza cases in Washington.

\section{Methods}

Timeliness and completeness of records received through the WA-HIE were evaluated. Basic characteristics and time trends of WA-HIE influenza hospitalizations (that is, records with ICD9 487-488 listed in the final diagnoses) between April 27, 2009-May 15, 2010 were compared with lab-confirmed influenza hospitalizations of eastern Washington residents reported through the WA notifiable condition reporting system (PHIMS). Overlap between the WA-HIE and PHIMS influenza cases was assessed by conducting a record-level comparison with a subset of WA-HIE and PHIMS influenza cases.

\section{Results}

For $90 \%$ of admissions into WA-HIE hospitals, the first record is transmitted 1 day after admission (range 0-26 days). One or more data elements were missing from $34 \%$ of records 28 days after admission. Of these incomplete records, 96\% were missing the final diagnoses.
Data for influenza hospitalizations from the WA-HIE do not differ significantly from data collected through PHIMS with regard to sex, age, and mortality (Table 1). The time series of influenza-related hospital admissions from the WA-HIE and PHIMS are highly correlated ( $r=0.97$, Figure 1$)$.

Table 1 Characteristics of influenza cases detected through WA-HIE and reported through PHIMS

\begin{tabular}{lccc}
\hline Case characteristic & $\begin{array}{c}\text { WA-HIE }(\mathrm{N}=375) \\
\mathrm{N}(\%)\end{array}$ & $\begin{array}{c}\text { PHIMS }(\mathrm{N}=489) \\
\mathrm{N}(\%)\end{array}$ \\
\hline Sex & & & \\
$\quad$ Male & $154(41.1)$ & $199(40.7)$ & 0.91 \\
Female & $221(58.9)$ & $290(59.3)$ & 0.91 \\
Age group (years) & & & \\
0-4 & $78(20.8)$ & $116(23.7)$ & 0.32 \\
5-24 & $83(22.1)$ & $114(23.3)$ & 0.69 \\
25-49 & $94(25.1)$ & $127(26.0)$ & 0.76 \\
$50-64$ & $77(20.5)$ & $85(17.4)$ & 0.24 \\
$65+$ & $43(11.5)$ & $47(9.6)$ & 0.38 \\
Deaths & $12(3.2)$ & $22(4.5)$ & 0.33 \\
\hline
\end{tabular}

${ }^{\text {a }} P$-value calculated using the $\chi^{2}$-test.

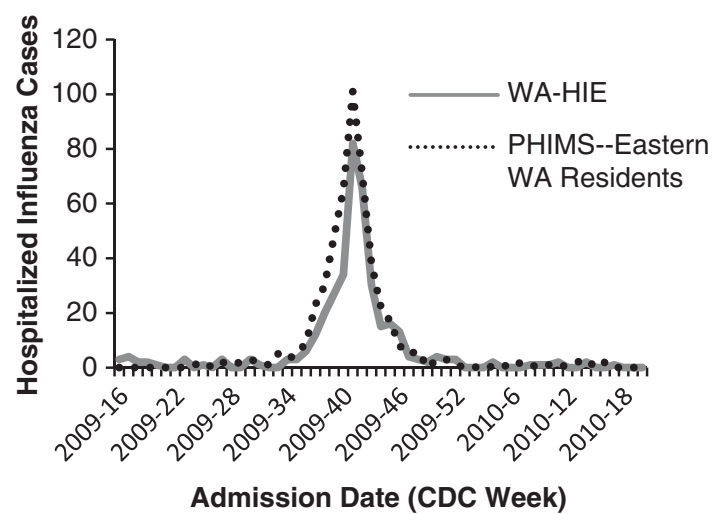

Figure 1 Time series of influenza cases detected through WA-HIE and reported through PHIMS.

open 2 Access This is an Open Access article distributed under the terms of the Creative Commons Attribution licence (http://creativecommons.org/licenses/by/2.5) OPEN - ACCESS which permits unrestricted use, distribution, and reproduction in any medium, provided the original work is properly cited. 
Of 305 WA-HIE influenza hospitalizations during OctoberDecember 2009, 136 (44.6\%) matched to a case reported in PHIMS.

\section{Conclusions}

WA-HIE data is an automated, relatively timely source of hospitalization information. Despite a lack of complete overlap between records reported through PHIMS and the WA-HIE, the WA-HIE data are representative of hospitalized influenza cases reported through PHIMS with respect to basic case characteristics and time trends.

\section{Future directions}

A prospective evaluation of hospitalized influenza cases is planned. In addition, the WA-HIE data elements are being expanding to allow for more in-depth data evaluation, detection of lab-confirmed influenza cases, assessment of illness severity, and evaluation of risk factors.

\section{Acknowledgements}

This paper was presented as an oral presentation at the 2010 International Society for Disease Surveillance Conference, held in Park City, UT, USA on 1-2 December 2010. 


\title{
Biosurveillance applications for resource-limited settings: open ESSENCE and ESSENCE desktop edition
}

\author{
JS Coberly ${ }^{1}$, RA Wojcik ${ }^{1}$, JF Skora ${ }^{1}$, AM Poku ${ }^{1}$, ZC Whitley ${ }^{1}$, TC Campbell ${ }^{1}$, CJ Hodanics ${ }^{1}$, SL Lewis ${ }^{1}$, and DL Blazes ${ }^{2}$ \\ ${ }^{1}$ The Johns Hopkins University Applied Physics Laboratory, Laurel, MD, USA; and ${ }^{2}$ Division of GEIS Operations, Armed Forces Health \\ Surveillance Center, Silver Spring, MD, USA \\ E-mail: jacqueline.coberly@jhuapl.edu
}

\section{Objective}

This paper describes the development and early implementation of two freely available electronic biosurveillance software applications: Open ESSENCE (OE), and ESSENCE Desktop Edition (EDE).

\section{Introduction}

More than a decade ago, in collaboration with the U.S. Department of Defense, the Johns Hopkins University Applied Physics Laboratory (JHU/APL) developed the Electronic Surveillance System for the Early Notification of Community-based Epidemics (Enterprise ESSENCE), ${ }^{1}$ which is currently used by federal, state and local health authorities in the US. As emerging infections will most likely originate outside of the US (for example, SARS) the application of electronic biosurveillance is increasingly important in resource limited areas. ${ }^{2}$ In addition, such systems help governments respond to the recently modified International Health Regulations. ${ }^{3}$ Leveraging the experience gained in the development of Enterprise ESSENCE, JHU/ APL has developed two freely available electronic biosurveillance systems suitable for use in resource-limited areas: OE and EDE.

\section{Methods}

Biosurveillance is needed at all levels of public health, the local health clinic, as well as city, regional and national health authorities. The availability of computers and trained personnel varies considerably among these entities. OE and EDE were designed with this disparity in mind. OE is a webbased application designed to run on a system with internet or intranet access. It provides web-based data entry and data visualization to multiple users. $\mathrm{EDE}$, on the other hand, is a stand-alone application designed to run on a single desktop or laptop computer. Both systems have much of the functionality found in the original Enterprise ESSENCE. They produce time series for the health data being analyzed, using both proprietary and open source alerting algorithms. Each also produces case-level data lists, pie and bar charts of any available variable, and alert or prevalence maps if appropriate geographical shape files are available. In addition, all of the user interface components in OE are internationalized. Addition of a language table created by the user allows OE to function in any language. Although EDE is currently only available with an English-user interface, it can read and present data in English, as well as in character-based languages, such as Thai. The most notable difference between Enterprise ESSENCE, OE and EDE is the data repositories. Enterprise ESSENCE has an established database schema and the data must conform to its schema. OE and EDE were designed to be more flexible. OE connects and adapts to the user's database and schema and EDE connects to databases and text files in various user formats.

\section{Results}

OE is currently being piloted in Peru by the Peruvian Navy and Army in conjunction with the U.S. Naval Medical Research Center Detachment (NMRCD). It was installed in 2009 and evaluation is ongoing. EDE is being piloted by the Cebu City Health Office in the Republic of the Philippines in conjunction with the Armed Forces Research Institute of Medical Sciences Virology Research Unit (PAVRU). Version 2.0 of EDE was installed early in 2010. An ongoing Simple Message Service (SMS) fever surveillance program will be used to pilot the application.

\section{Conclusions}

OE and EDE are freely available electronic biosurveillance applications, designed to facilitate surveillance at all levels of public health infrastructure. OE is web-based, and requires internet access for functionality, whereas EDE is a standalone desktop application designed for local users and emergency situations. Both applications provide an electronic surveillance capability, which can help countries conform to IHR 2005 regulations.

\section{Acknowledgements}

This paper was presented as a poster at the 2010 International Society for Disease Surveillance Conference, held in Park City, UT, USA on 1-2 December 2010.

open 2 access This is an Open Access article distributed under the terms of the Creative Commons Attribution licence (http://creativecommons.org/licenses/by/2.5) OPEN - ACCESS which permits unrestricted use, distribution, and reproduction in any medium, provided the original work is properly cited. 


\section{References}

1 Lombardo JS, Ross DA. Disease surveillance: a public health priority. In: Lombardo JS, Buckeridge DL (eds). Disease surveillance: a public health informatics approach. John Wiley \& Sons, Inc: Hoboken, 2007.

2 Heymann D. The international response to the 2003 SARS outbreak. Philos Trans R Soc Lond B Biol Sci 2004;359:1127-9.

3 Rodier G. New rules on international public health security. Bull World Health Organ 2007;85:428-30. 


\title{
Relationship between neighborhood poverty and emergency department utilization for fever/flu syndrome
}

\author{
K Desy, K Purviance, M Cartter, and JL Hadler \\ Connecticut Department of Public Health, Epidemiology and Emerging Infections Program, Hartford, CT, USA \\ E-mail: kristen.desy@ct.gov
}

\section{Objective}

To describe the relationship between neighborhood poverty and emergency department visits for fever/flu syndrome illnesses reported through the Connecticut Hospital Emergency Department Syndromic Surveillance (HEDSS) system.

\section{Introduction}

The HEDSS system was implemented in 2004 to monitor disease activity. ${ }^{1}$ In all, 18 of 32 emergency departments (ED) and 1 urgent care clinic provide data. Chief complaints are routinely categorized into eight syndromes. The fever/flu syndrome is used for early detection and monitoring of influenza in the community. ${ }^{2}$

Area-based measures, such as zip code, enable linkage to area-based socioeconomic census data. Neighborhood poverty, defined as the percentage of persons living below the federal poverty level in a geographic area, predicts a wide range of disease outcomes. ${ }^{3}$

\section{Methods}

HEDSS data were categorized into 6-week periods of maximal influenza activity: baseline (9/06-10/18/2008), 2008-2009 seasonal, spring 2009 H1N1 and fall 2009 H1N1. Neighborhood poverty was categorized as low $(<5 \%$ below federal poverty level), medium-low $(5-<10 \%)$, medium-high $(10-<20 \%)$, and high $(\geqslant 20 \%)$ based on zipcode. Chi-square for trend was calculated for age and poverty-stratified percentages of total ED visits for fever/flu for each time period. The percentage increase over baseline in ED visits for fever/flu was calculated by neighborhood poverty level (Figure 1).

\section{Results}

There were a total of 4051 fever/flu visits at baseline, 8369 during the seasonal peak, 11426 during the 2009 spring H1N1 peak, and 14057 during the fall 2009 H1N1 peak. During each of these periods, the percentage of ED visits that were fever/flu increased with increasing poverty level
$\left(P<0.0001, \chi^{2}\right.$ for trend). The strength of the association was similar at baseline and for each influenza period and for each age group except $\geqslant 65$ years for which no relationship was seen between poverty and ED utilization for fever/flu.

The percentage increase in ED visits for fever/flu compared with baseline was similar for all poverty levels and age groups in each influenza season, with the exception of 5-17 year olds who had significantly higher rates of ED utilization with increasing poverty level during the spring $2009 \mathrm{H} 1 \mathrm{~N} 1$ wave $(P<0.0001)$.

\section{Conclusions}

There is a strong and consistent association between neighborhood poverty and ED visits for fever/flu compared with all other syndromes combined. The strength of association is similar for fever/flu illnesses at baseline, during seasonal flu, and during each of the H1N1 waves. The percentage increase in ED visits for fever/flu during each flu season was similar for all poverty levels. If visiting the ED makes one more likely to be admitted as an inpatient than not visiting the ED, this could partially explain the increased incidence of hospitalization seen in persons in higher poverty neighborhoods. Efforts to reduce ED visits for

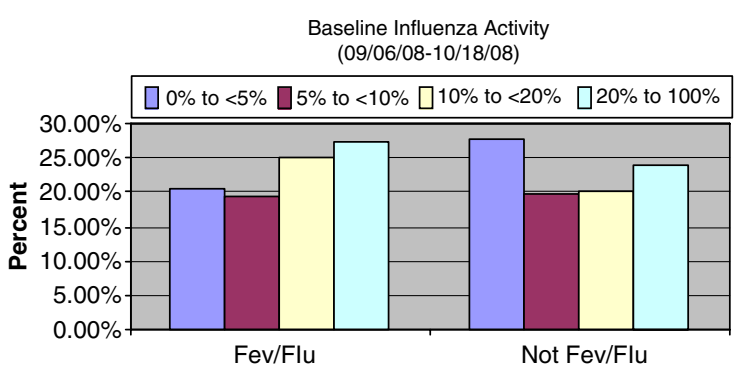

Figure 1 At baseline, ED utilization for fever/flu syndrome was associated with increasing neighborhood poverty. This was not true of non-fever/flu visits. Similar patterns in ED utilization were observed during the other influenza activity periods.

open 2 access This is an Open Access article distributed under the terms of the Creative Commons Attribution licence (http://creativecommons.org/licenses/by/2.5) OPEN - access which permits unrestricted use, distribution, and reproduction in any medium, provided the original work is properly cited. 
fever/flu (for example, vaccination, education) should be targeted to higher poverty neighborhoods. Additional study is needed to determine why persons from higher-poverty neighborhoods seem to be more likely to visit the ED for fever/flu syndrome.

\section{Acknowledgements}

This paper was presented as an oral presentation at the 2010 International Society for Disease Surveillance Conference, held in Park City, UT, USA on 1-2 December 2010.

\section{References}

1 Dembek Z, Carley K, Hadler J. Guidelines for constructing a statewide hospital syndromic surveillance network. MMWR 2005;54 (Suppl): 21-4.

2 Heffernan R, Mostashari F, Das D, Karpati A, Kulldorff M, Weiss D. Syndromic surveillance in public health practice, New York City. EID 2004;10:858-64.

3 Krieger N, Chen JT, Waterman PD, Rehkopf DH, Subramanian SV. Race/ethnicity, gender, and monitoring socioeconomic gradients in health: a comparison of area-based socioeconomic measures-The Public Health Disparities Geocoding Project. Am J Public Health 2003;93:1655-71. 


\title{
A comparison of the fever-flu syndrome category with the SC ILINet surveillance system in South Carolina: 2009-2010 influenza season
}

\author{
H Dhotre, C Springs, D Drociuk, and YE Cheng \\ Division of Acute Disease Epidemiology (DADE), SC Department of Health and Environmental Control (SC DHEC), Columbia,SC, USA \\ E-mail: dhotrehc@dhec.sc.gov
}

\begin{abstract}
Objective
This paper compares the South Carolina Aberration Alerting Network's (SCAAN) hospital-based fever-flu syndrome category with the South Carolina Outpatient Influenza-like Illness Network (ILINet) provider surveillance system. This is the first comparison of South Carolina's syndromic surveillance SCAAN data with ILINet data since SCAAN's deployment.
\end{abstract}

\section{Introduction}

SCAAN is a collaborative network of syndromic systems within South Carolina. Currently, SCAAN contains the following data sources: SC Hospital Emergency Department (ED) chief-complaint data, Poison Control Center call data, Over-the-Counter pharmaceutical sales surveillance, and CDC's BioSense biosurveillance system. ILINet is collaboration between the Centers for Disease Control, state health departments and health care providers. ILINet is one of several components of SC's influenza surveillance. ${ }^{1,2}$

\section{Methods}

The inclusion criteria for this study were any individuals who visited a hospital facility or an ILI provider in South Carolina from 25 April 2009 to 26 June 2010.

As of July 2010, a total of 14 hospital facilities are enrolled in the SCAAN system. There is at least one hospital facility reporting ED data in five of the eight public health regions of the state.

Seventy South Carolina providers were enrolled in ILINet during the 2009-2010 season. Provider enrollment encompassed all eight DHEC public health regions.

The fever-flu syndrome category and SC ILI surveillance share the same definition: fever $\left(\geqslant 100^{\circ} \mathrm{F}\right)$ and cough and/or sore throat (in the absence of a known cause other than influenza).

The weekly fever-flu percentage was calculated by dividing the total number of ED visits with a fever-flu chief- complaint seen in all hospitals by the total number of ED visits seen by all hospitals.

The weekly state ILI percentage was calculated by dividing the total number of patients seen with ILI by the total number of patients seen.

The Pearson's correlation coefficient test was performed in SAS v.9.2 to assess the strength of association between the fever-flu syndromic surveillance and ILI surveillance. Additional analysis will be conducted to compare both surveillance methods geographically.

\section{Results}

Figure 1 shows the weekly fever-flu and ILI percentage from 25 April 2009 through 26 June 2010. There was an initial increase in ILI-related visits to the hospital ED near the end of April 2009. This was also the same time period the H1N1 novel influenza media releases were occurring nationally and statewide.

A sharp increase in the number of visits related to ILI was evident around the beginning of September 2009 through the end of October 2009. During this time period, the mean for fever-flu and ILI percentage was $9.29 \%$

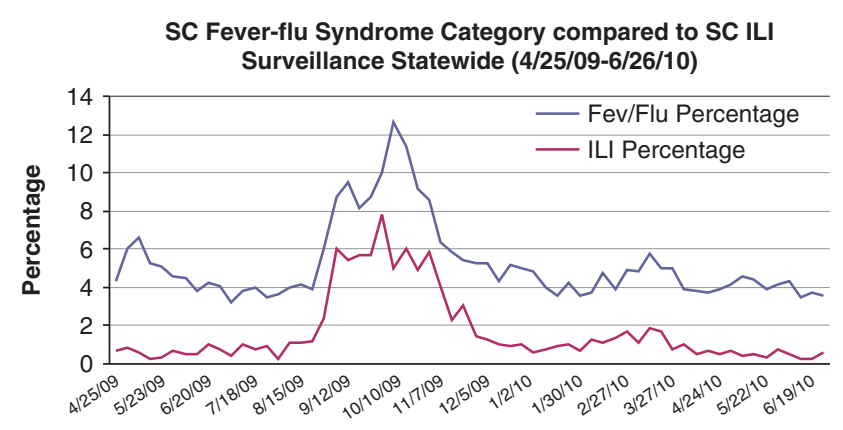

MMWR Week

Figure 1 South Carolina's weekly fever-flu and ILI percentage from 25 April 2009 to 26 June 2010. This is an Open Access article distributed under the terms of the Creative Commons Attribution licence (http://creativecommons.org/licenses/by/2.5)
which permits unrestricted use, distribution, and reproduction in any medium, provided the original work is properly cited. 
(range: 6.05-12.67\%) and 5.48\% (range: 2.40-7.77\%), respectively. A peak in the ILI percentage occurred slightly before the peak in fever-flu percentage. Excluding the peak period (September-October), the mean for fever-flu and ILI percentage was $4.44 \%$ (range: 3.22-6.66\%) and 0.96\% (range: $0.24-4.1 \%$ ), respectively. There was a high correlation ( $r=0.891)$ between fever-flu and ILI percentages. Additional correlation analysis will be conducted to account for the geographic distribution of the two data sources (hospital EDs and provider clinics).

\section{Conclusions}

On the basis of these findings, the SCAAN fever-flu syndrome category offers an additional surveillance tool to the existing ILI surveillance system. It is useful to understand the population of SC residents who visit the hospital ED versus a private provider clinic for ILI-related issues. A phasing-out of ILI surveillance for the more reliable hospital ED fever-flu surveillance (daily automated analysis) may be a consideration once more hospitals join the SCAAN system.

\section{Acknowledgements}

This paper was presented as a poster at the 2010 International Society for Disease Surveillance Conference, held in Park City, UT, USA, on 1-2 December 2010.

\section{References}

1 Marsden-Haug N, Foster VB, Gould PL, Elbert E, Wang H, Pavlin JA. Code-based syndromic surveillance for influenza like illness by International Classification of Diseases, Ninth Revision. Emerg Infect Dis 2007;2:207 (http://www.cdc.gov/EID/content/13/ 2/207).

2 The Centers for Disease Control and Prevention. The Early Aberration Reporting System. http://emergency.cdc.gov/surveillance/ears/. 


\title{
Can all-cause school absenteeism be used to optimize the timing of school closure in response to pandemic influenza?
}

\author{
JR Egger ${ }^{1,2,3}$, AG Hoen ${ }^{1,2,3}$, E Wilson ${ }^{1,2,3}$, JS Brownstein ${ }^{1,2,3}$, DL Buckeridge ${ }^{1,2,3}$, and KJ Konty ${ }^{1,2,3}$ \\ ${ }^{1}$ New York City Department of Health and Mental Hygiene, New York, NY, USA; ${ }^{2}$ Children's Hospital Boston and Harvard Medical School, \\ Boston, MA, USA; and ${ }^{3}$ McGill Clinical and Health Informatics, McGill University, Montreal, QC, Canada \\ E-mail: jegger@health.nyc.gov
}

\section{Objective}

To determine the optimal pattern in school-specific all-cause absenteeism for use in informing school closure related to pandemic influenza.

\section{Introduction}

School closure has long been proposed as a non-pharmaceutical intervention in reducing the transmission of pandemic influenza. ${ }^{1}$ Children are thought to have high transmission potential because of their low immunity to circulating influenza viruses and high contact rates. In the wake of pandemic (H1N1) 2009, simulation studies suggest that early and sustained school closure might be effective at reducing community-wide transmission of influenza. ${ }^{2,3}$ Determining when to close schools once an outbreak occurs has been difficult. Influenza-related absentee data from Japan were previously used to develop an algorithm to predict an outbreak of influenza-related absenteeism. ${ }^{4}$ However, the cause of absenteeism is frequently unavailable, making application of this model difficult in certain settings. For this study, we aimed to evaluate the potential for adapting the Japanese algorithm for use with all-cause absenteeism, using data on the rate of influenza-related nurse visits in corresponding schools to validate our findings.

\section{Methods}

Data consisted of the daily count of all-cause absenteeism and school nurse visits for fever/flu syndrome in 1206 public schools in New York City from 6 September 2005 to 26 June 2009. A model-adjusted school-specific z-score was calculated for both absenteeism and ILI for each day from 5 September 2006 to 28 June 2009. The z-score was calculated by dividing the model residual by the school-specific standard deviation of the outcome. Both models adjusted for day of week, whether the preceding or proceeding day was a holiday, school type (elem., middle, high), school day and sine and cosine terms to account for seasonality.

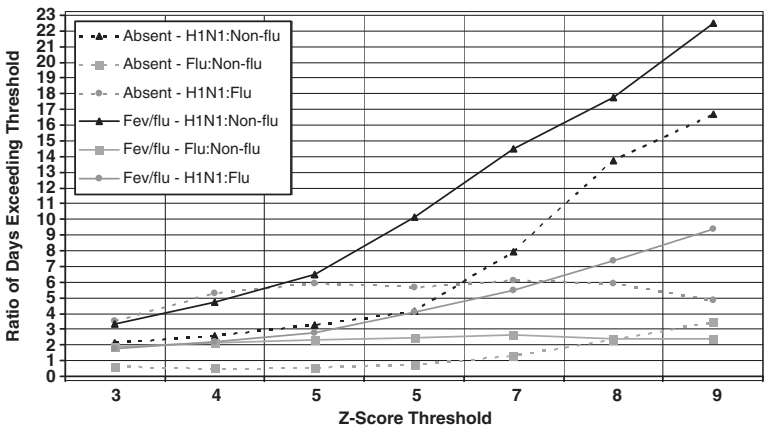

Figure 1 The ratio of the proportion of days exceeding various z-score thresholds, comparing the seasonal, $\mathrm{pH} 1 \mathrm{~N} 1$, and non-flu periods.

\section{Results}

The ratio of the proportion of z-scores higher than a given threshold for the non-flu period, seasonal flu period and the pH1N1 2009 period (April 20-June 19, 2009) are shown in the Figure 1. As expected, the ratio of high fever/flu z-scores during the $\mathrm{pH} 1 \mathrm{~N} 1$ period to non-flu periods is greater than one. This pattern is similar for high absentee z-scores during the pH1N1 period compared with the non-flu period; however, the ratio of absentee z-scores during the seasonal flu period compared with the non-flu period is below one for z-scores of six or less. This trend reverses itself for z-scores greater than six. In fact, for both absenteeism and fever/flu, the ratio tends to increase with an increasing threshold cutoff.

\section{Conclusions}

Preliminary results indicate that for moderately high absenteeism (z-score $<6$ ) there is little variation between seasonal and non-seasonal flu periods. However, high absenteeism (z-score $>6$ ) occurs more frequently during periods of flu transmission, suggesting that a high absentee threshold may be needed to predict a flu outbreak. Further work will involve determining the optimal temporal pattern This is an Open Access article distributed under the terms of the Creative Commons Attribution licence (http://creativecommons.org/licenses/by/2.5)
open 2 access permits unrestricted use, distribution, and reproduction in any medium, provided the original work is properly cited. 
and threshold levels in absenteeism that best predicts both high absenteeism and fever/flu visits. Results of this work will help determine the relative utility of tracking all-cause school absenteeism for use in school-based influenza surveillance.

\section{Acknowledgements}

This paper was presented as an oral presentation at the 2010 International Society for Disease Surveillance Conference, held in Park City, UT, USA on 1-2 December 2010.

\section{References}

1 Cauchemez S, Ferguson NM, Wachtel C, Tegnell A, Saour G, Duncan B, et al. Closure of schools during an influenza pandemic. Lancet Infect Dis 2009;9:473-81.

2 Halder N, Kelso JK, Milne GJ. Analysis of the effectiveness of interventions used during the $2009 \mathrm{~A} / \mathrm{H} 1 \mathrm{~N} 1$ influenza pandemic. BMC Public Health 2010;10:168.

3 Lee BY, Brown ST, Cooley P, Potter MA, Wheaton WD, Burke DS, et al. Simulating school closure strategies to mitigate an influenza epidemic. J Public Health Manag Pract 2010;16(3):252-61.

4 Sasaki A, Hoen AG, Ozonoff A, Suzuki H, Tanabe N, Seki N, et al. Evidence-based tool for triggering school closures during influenza outbreaks Japan. Emerg Infect Dis 2009;15:1841-3. 


\title{
Evaluating the utility of school absenteeism during the 2009-2010 influenza season in Los Angeles County
}

\author{
C Faustino, P Araki, E Kajita, M Jones, and B Hwang
}

Los Angeles Department of Public Health (LACDPH), Acute Communicable Disease Control Unit (ACDC), Automated Disease Surveillance Section (ADSS), Los Angeles, CA, USA

E-mail: cfaustino@ph.lacounty.gov

\section{Objective}

To evaluate the utility of school absenteeism surveillance data in Los Angeles County during the 2009-2010 influenza season.

\section{Introduction}

It has been postulated that school absenteeism, a nontraditional surveillance data source, may allow for early detection of disease outbreaks, particularly among schoolaged children who may not seek emergency medical attention. Although a New York City-based study showed moderate utility of school absenteeism in biosurveillance, ${ }^{1}$ no study to date has been reported on school absenteeism in
Los Angeles County, which contains the second largest school district in the US. ${ }^{2}$

\section{Methods}

School absenteeism data on school attendance are negativebased and completed by teachers via the online student information system; once per day for elementary schools, once per period for middle/high schools. Any final corrections to attendance are made at the end of the school day through an administrative portal. School absenteeism data are received by LACDPH in near real-time on a biweekly basis via SFTP.

For the purposes of this study, school absenteeism data were available from 140 elementary, middle, and high schools, during

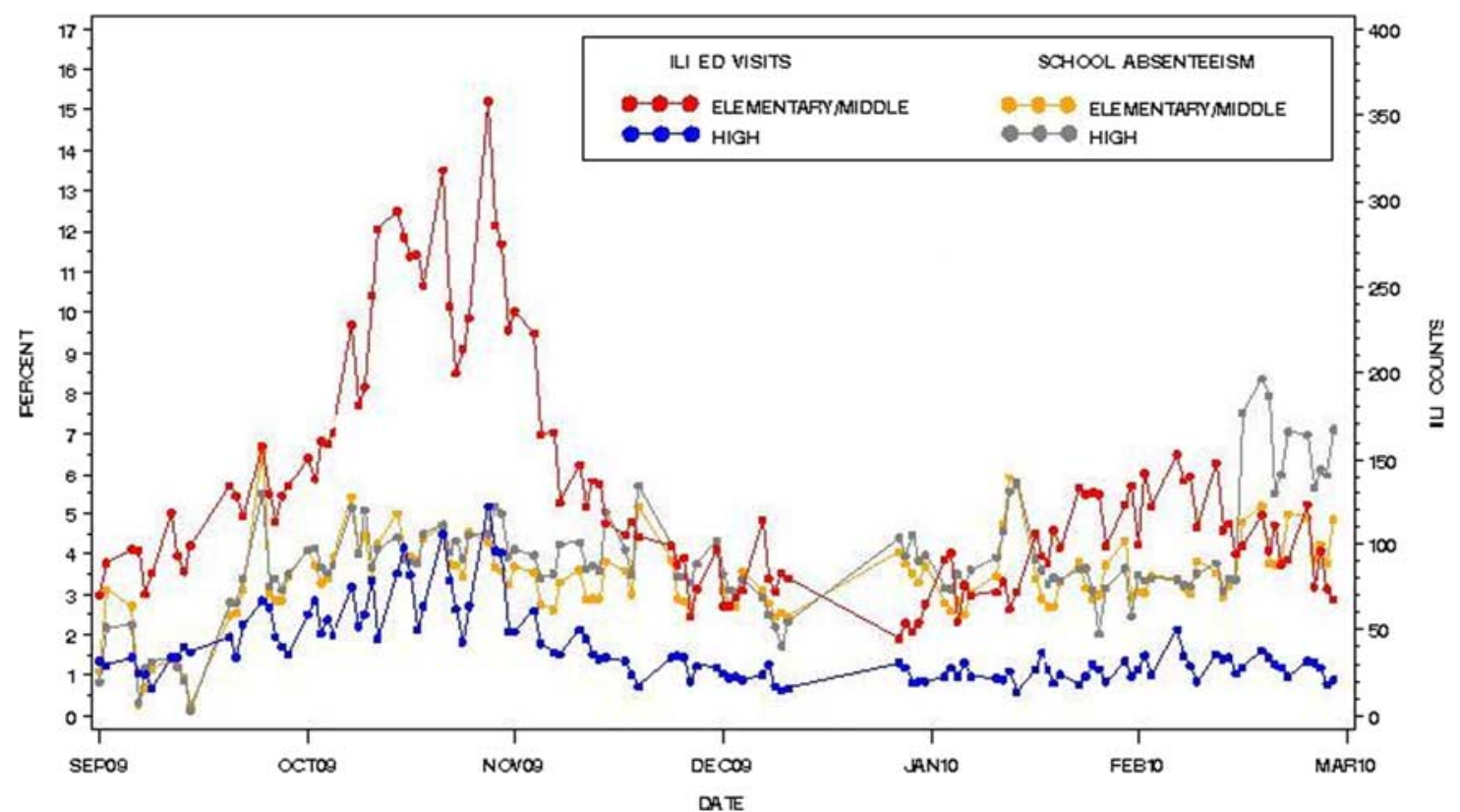

Figure 1 Percent School Absenteeism and ILI ED visits by School Age Groups in Los Angeles County.

This is an Open Access article distributed under the terms of the Creative Commons Attribution licence (http://creativecommons.org/licenses/by/2.5) OPEN - ACCESS which permits unrestricted use, distribution, and reproduction in any medium, provided the original work is properly cited. 
the period from 1 September 2009 through 28 February 2010. A retrospective analysis was conducted to examine the correlations between school absenteeism and two other ADSS influenza surveillance systems: (1) influenza-like illness emergency department (ILI ED) visits by ages 5-17 and (2) over-the-counter cough/cold medications and thermometer sales.

\section{Results}

During the study period, mean percent absenteeism was $3.28 \%$ for elementary/middle schools and $3.55 \%$ for high schools $(p=0.06)$. Figure 1 shows the number of ILI ED visits, which peaked in early November for both elementary/ middle and high school groups. In contrast, however, school absenteeism peaked in late September/early October for both groups. Compared with ILI ED visits in elementary/middle school-aged (5-13 years) and high school-aged (14-17 years) children, school absenteeism showed a weak correlation ( $r=0.38, p<0.001 ; r=0.33, p<0.001$, respectively). Also, neither cough/cold medication sales nor thermometer sales showed strong correlations with either elementary/middle or high school absenteeism (data not shown).

\section{Conclusions}

We found no significant positive correlations of school absenteeism in elementary/middle school and high school groups, with either ILI ED visits or over-the-counter medica- tion sales in Los Angeles County during the 2009-2010 influenza season. Interpreting medical outcomes/trends from a non-traditional source like school absenteeism is challenging, and continued investigation is warranted before determining its role in biosurveillance. Moreover, school absenteeism could serve to help assess the need of school closures during countywide disease outbreaks. However, further assessment of current data capture methods and quality of school absenteeism data within Los Angeles County are necessary to evaluate the utility of absenteeism in early event detection.

\section{Acknowledgements}

This paper was presented as a poster at the 2010 International Society for Disease Surveillance Conference, held in Park City, UT, USA, on 1-2 December 2010.

\section{References}

1 Besculides M, Heffernan R, Mostashari F, Weiss D. Evaluation of school absenteeism data for early outbreak detection. New York City. BMC Public Health 2005;5:105.

2 Snyder TD, Dillow SA, Hoffman CM. Digest of Education Statistics 2008 (NCES 2009-020). National Center for Education Statistics, Institute of Education Sciences, US Department of Education: Washington, DC, 2009, pp 130-5. http://nces.ed.gov/pubs2009/ 2009020.pdf (retrieved 13 October 2010). 


\title{
Comparison of the US outpatient influenza-like illness surveillance network and the Google Flu Trends from 2008 to 2010
}

\author{
A Fowlkes ${ }^{1}, \mathrm{M} \mathrm{Mohebbi}{ }^{2}, \mathrm{~S}$ Cook $^{2}, \mathrm{C} \mathrm{Conrad}^{2}, \mathrm{~L} \mathrm{Brammer}{ }^{1}$, and L Finelli ${ }^{1}$ \\ ${ }^{1}$ Influenza Division, Centers for Disease Control and Prevention, Atlanta, GA, USA; and ${ }^{2}$ Google.org, New York City, NY, USA \\ E-mail: ahl4@cdc.gov
}

\section{Objective}

This paper compares estimates of influenza-like illness (ILI) activity with estimates from the Centers for Disease Control's ILINet from October 2008 through March 2010.

\section{Introduction}

http://Google.org developed a regression model that used the volume of influenza-related search queries best correlated with the proportion of outpatient visits related to ILI model to estimate the level of ILI activity. ${ }^{1}$ For calibration, the model used ILINet data from October 2003 to 2009, which report weekly ILI activity as the percentage of patient visits to health care providers for ILI from the total number patient visits for the week. Estimates of ILI in 121 cities were added in January 2010.

\section{Methods}

For the 2008-2009 and 2009-2010 influenza seasons, we used ILINet data to compare the weekly percentage of outpatient ILI visits with the web query-based model estimates of ILI from Google Flu Trends. Data from Google Flu Trends are publicly available through the website. The time period for evaluation was parsed according to the emergence of pandemic influenza A (H1N1) virus (pH1N1): 'pre-pandemic' was defined as October 2008 through March 2009, 'wave one' as April through July 2009, and 'wave two'

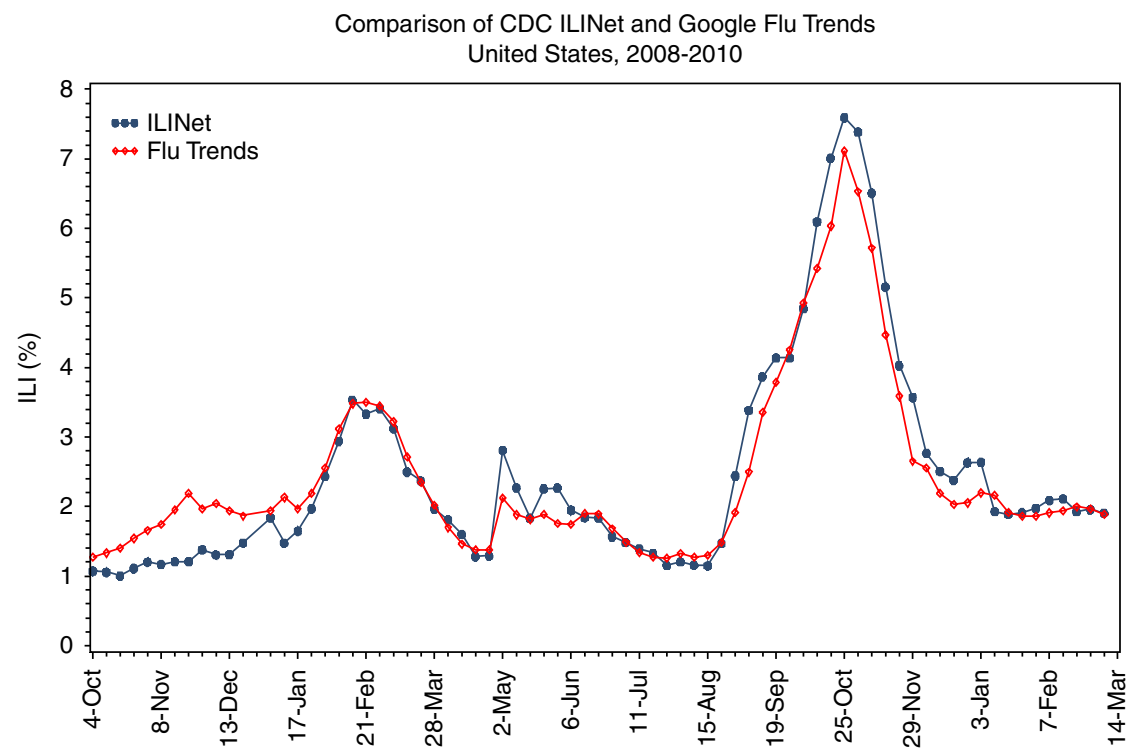

Figure 1 Comparison of Google Flu Trends estimates of influenza-like illness (red) with ILINet estimates from October 2008 to March 2010.

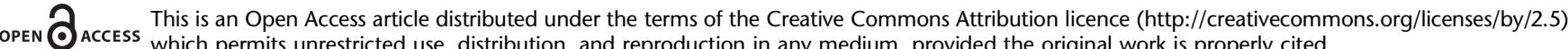
OPEN ACCESS which permits unrestricted use, distribution, and reproduction in any medium, provided the original work is properly cited. 
as August 2009 through April 2010. A correlation coefficient was calculated for the nation, US Department of Health and Human Resources-defined regions, and states, and for 47 major metropolitan areas. Correlations below zero were displayed as zero.

\section{Results}

From October 2008 through April 2010, all national and regional ILI percentage estimates reported by ILINet and Google Flu Trends correlated highly (Figure 1). During each of the study time periods, the median correlation was 0.93 for national and regional comparisons. In the state-specific comparisons, the median correlation during the pre-pandemic season was 0.82 , including two states with zero correlation (range 0-0.94). During wave one the median state correlation was 0.65 , including four states with zero correlation (range 0-0.99). During wave two the median state correlation was 0.94 (range 0.68-0.99). In the major metropolitan areas, the median city correlation was 0.73 (range 0-0.95) during the pre-pandemic period, 0.48 (range 0-0.93) during wave one, and 0.89 (range 0.18-0.99) during wave two.

\section{Conclusions}

During periods of widespread influenza circulation, the percentage of ILI-related outpatient visits determined by ILINet and estimated by Google Flu Trends correlated well for national, regional, state and many major metropolitan-area levels. After the introduction of $\mathrm{pH} 1 \mathrm{~N} 1$, but before widespread circulation, state and metropolitan area estimates of ILI activity were less correlated. Google's experimental estimates of ILI activity in major metropolitan areas correlated well with provider-based surveillance data, but are less well correlated than the more robust national and regional data.

\section{Acknowledgements}

This paper was presented as a poster at the 2010 International Society for Disease Surveillance Conference, held in Park City, UT, USA, on 1-2 December 2010.

\section{Reference}

1 Ginsberg J, Mohebbi MH, Patel RS, Brammer L, Smolinski MS, Brilliant L. Detecting influenza epidemics using search engine query data. Nature 2009;457:1012-14. 


\title{
Using influenza rapid test positivity as an early indicator for the onset of seasonal influenza
}

\author{
LH Gren ${ }^{1}$, SC Alder ${ }^{1}$, EA Joy ${ }^{1}$, JL Lyon ${ }^{1}$, CA Porucznik ${ }^{1}$, and C) Staes ${ }^{2}$ \\ ${ }^{1}$ Departments of Family and Preventive Medicine, University of Utah School of Medicine, Salt Lake City, UT, USA; and ${ }^{2}$ Department \\ of Biomedical Informatics, University of Utah School of Medicine, Salt Lake City, UT, USA \\ E-mail: lisa.gren@hsc.utah.edu
}

\section{Objective}

The objective of this study is to describe the use of point-ofcare rapid influenza testing in an outpatient, setting for the identification of the onset of influenza in the community.

\section{Introduction}

Influenza causes significant morbidity and mortality, with attendant costs of roughly $\$ 10$ billion for treatment and up to $\$ 77$ billion in indirect costs annually. ${ }^{1-3}$ The Centers for Disease Control and Prevention conducts annual influenza surveillance, and includes measures of inpatient and outpatient influenza-related activity, disease severity, and geographic spread. ${ }^{4}$ However, inherent lags in the current methods used for data collection and transmission result in a one to two weeks delay in notification of an outbreak via the Centers for Disease Control and Prevention's website. ${ }^{5}$ Early notification might facilitate clinical decision-making when patients present with acute respiratory infection during the early stages of the influenza outbreak.

In the United States, the influenza surveillance season typically begins in October and continues through May. The Utah Health Research Network has participated in Centers for Disease Control and Prevention's influenza surveillance since 2002, collecting data on outpatient visits for influenza-like illness (ILI, defined as fever of $100 \mathrm{~F}$ or higher with either cough or sore throat). Over time, Utah Health Research Network has moved from data collection by hand to automated data collection that extracts information from discrete fields in patients' electronic health records.

We used statistical process control to generate surveillance graphs of ILI and positive rapid influenza tests, using data available electronically from the electronic health records.

\section{Methods}

Rapid influenza tests were introduced into Utah Health Research Network in December 2003. We extracted data from the clinical data warehouse for influenza seasons 20042005 through 2007-2008. ILI patients were those who: (1) had a measured temperature at the visit of at least 100F, and (2) had a 'reason for visit' field that contained either cough or sore throat. Regardless of ILI status, any patient who had a positive influenza rapid test was also identified.

The daily proportion of patients with ILI or with a positive rapid influenza test was graphed using statistical process control charts. Using the Western electric rules, we defined both the epidemic onset of the influenza outbreak and an early alert signal. We compared the presumed notification date for the early alert signal with the usual notification system in Utah that relies on data collected for Centers for Disease Control and Prevention's influenza surveillance to determine the timeliness of rapid test positivity surveillance.

\section{Results}

The peaks for ILI and rapid test positivity coincided in time. An early alert was identified in all four seasons using rapid test positivity, although no early alert was clearly identified for ILI in any season. The early alert for rapid test positivity occurred a median of 16 days before the epidemic onset. Assuming dissemination by email within two days of the alert, Utah Health Research Network clinicians could be notified of the start of the influenza outbreak about nine days earlier than ILI surveillance alerts posted to the Utah Department of Health website.

\section{Conclusions}

Influenza rapid test positivity provided an early alert, 16 days before the epidemic onset, and earlier than traditional surveillance for ILI. This early awareness could guide clinician decisions regarding diagnostic testing, prescription of antivirals, and counseling their patients on preventive behaviors. This is an Open Access article distributed under the terms of the Creative Commons Attribution licence (http://creativecommons.org/licenses/by/2.5)
open 2 access
which permits unrestricted use, distribution, and reproduction in any medium, provided the original work is properly cited. 


\section{Acknowledgements}

This paper was presented as an oral presentation at the 2010 International Society for Disease Surveillance Conference, held in Park City, UT, USA, on 1-2 December 2010.

\section{References}

1 Thompson WW, Shay DK, Weintraub E, Brammer L, Cox N, Anderson $\mathrm{LJ}$, et al. Mortality associated with influenza and respiratory syncytial virus in the United States. JAMA 2003;289:179-86.
2 Thompson WW, Shay DK, Weintraub E, Brammer L, Bridges CB, Cox NJ, et al. Influenza-associated hospitalizations in the United States. JAMA 2004;292:1333-40.

3 Molinari NA, Ortega-Sanches IR, Messonnier ML, Thompson WW, Wortley PM, Weintraub E, et al. The annual impact of seasonal influenza in the US: measuring disease burden and costs. Vaccine 2007;25:5086-96.

4 Centers for Disease Control and Prevention. Flu activity and surveillance. http://www.cdc.gov/flu/weekly/fluactivity.htm.

5 Gren LH. Outpatient influenza surveillance: bridging the gap between clinical medicine and public health. [dissertation]. [Salt Lake City]: University of Utah; 2010. 88p. 


\title{
Creating a shared epidemiologic vocabulary: lessons from the former Soviet Union
}

\author{
A Grigoryan' ${ }^{1}$, C Clarke ${ }^{2}$, T Chumachenko ${ }^{3}$, and B Smoak ${ }^{2}$ \\ ${ }^{1}$ Office of Surveillance, Epidemiology and Laboratory Services, Centers for Disease Control and Prevention, Atlanta, GA, USA; \\ ${ }^{2}$ Division of Preventive Medicine, Walter Reed Army Institute of Research, Silver Spring, MD, USA; and ${ }^{3}$ Kharkiv National Medical University, \\ Kharkov, Ukraine \\ E-mail: AGrigoryan@cdc.gov
}

\section{Objective}

The objective of this study is to describe the development of a multilingual lexicon of epidemiology, which is needed for improved communication in public health surveillance internationally.

\section{Introduction}

As part of the United States Department of Defense strategy to counter biological threats, the Defense Threat Reduction Agency's biological threat reduction program is enhancing the capabilities of countries in the former Soviet Union (FSU) to detect, diagnose, and report endemic and epidemic, manmade or natural cases of especially dangerous pathogens. During these engagements, it is noted that Western-trained and Soviet-trained epidemiologists have difficulty, beyond that of simple translation, in exchanging ideas.

Before 1991, infectious disease surveillance in the FSU was centrally planned in Moscow. The methodologies of infectious disease surveillance and data analysis have remained almost unaltered since this time in most nations of the FSU. Vlassov $^{1}$ describes that FSU physicians and other specialists are not taught epidemiology as it is understood in the West. The Soviet public health system and the scientific discipline of epidemiology developed independently of that of other nations. Consequently, many fundamental Soviet terms and concepts lack simple correlates in English and other languages outside the Soviet sphere; the same is true when attempting to translate from English to Russian and other languages of the FSU. Systematic review of the differences in FSU and Western epidemiologic concepts and terminology is therefore needed for international public health efforts, such as disease surveillance, compliance with International Health Regulations 2005, pandemic preparedness, and response to biological terrorism. A multi-language reference in the form of a dictionary would greatly improve mutual comprehension among epidemiologists in the West and the FSU.

\begin{abstract}
Methods
Following an extensive search of the Russian and English literature by a working group of Western and FSU epidemiologists, we created a matrix containing English and Russian definitions of key epidemiologic terms found in FSU and Western epidemiology manuals and dictionaries, such as $A$ Dictionary of Epidemiology ${ }^{2}$ and other sources. Particular emphasis was placed on terms relating to infectious disease surveillance, analysis of surveillance data, and outbreak investigation. In order to compare the definitions of each term and to elucidate small or large differences in usage, all definitions were translated into English and Russian so that the definitions could be compared side by side and discussed by the working group.
\end{abstract}

\section{Results \\ Three hundred and fifty-four terms from 13 English and 12 Russian sources were chosen for inclusion. Review of the definitions showed that many terms used in biosurveillance and infectious disease public health practice by FSU and Western-trained epidemiologists are used differently, and some concepts are lacking altogether in the Russian or English literature.}

\section{Conclusions}

Epidemiologic concepts and definitions significantly differed in the FSU and Western literature. To improve biosurveillance and international collaboration, recognition of these differences must occur, and where possible, agreement on standard definitions. Where agreement is not possible, precisely worded definitions illuminating differences in meaning and usage are essential. Implications for biosurveillance and public health practice will be discussed in the presentation and paper. This bilingual dictionary is expected to form the basis for trilingual dictionaries (Russian, English, and other languages

open 2 access This is an Open Access article distributed under the terms of the Creative Commons Attribution licence (http://creativecommons.org/licenses/by/2.5) OPEN ACCESS which permits unrestricted use, distribution, and reproduction in any medium, provided the original work is properly cited. 
of the FSU, such as Armenian, Azeri, Georgian and Ukrainian).

\section{Acknowledgements}

This paper was presented as a poster at the 2010 International Society for Disease Surveillance Conference, held in Park City, UT, USA, on 1-2 December 2010 .
The views expressed are those of the authors and should not be construed to represent the positions of the Department of the Army or Department of Defense.

\section{References}

1 Vlassov V. Is there epidemiology in Russia? J Epidemiol Community Health 2000;54:740-4.

2 Last JD. A Dictionary of Epidemiology, 4th edn. Oxford University Press: NY, 2001. 


\title{
Effect of expanded recommendations for pediatric seasonal influenza vaccination: an international comparison
}

\author{
AG Hoen $^{1,2}$, DL Buckeridge ${ }^{2}, \mathrm{KML}$ Charland $^{1,2}, \mathrm{KD} \mathrm{Mandl}^{1}$, and JS Brownstein ${ }^{1}$ \\ ${ }^{1}$ Children's Hospital Boston and Harvard Medical School, Boston, MA, USA; and ${ }^{2}$ McGill Clinical and Health Informatics, McGill University, \\ Montreal, Quebec, Canada \\ E-mail: anne.gatewood-hoen@childrens.harvard.edu
}

\section{Objective}

The objective of this study is to estimate the effect of expanding recommendations for routine seasonal influenza vaccination to include 24-59-month-old children.

\section{Introduction}

Seasonal influenza epidemics are responsible for over 200,000 hospitalizations in the United States per year, and 39,000 of them are in children. ${ }^{1,2}$ In the United States, the Advisory Committee on Immunization Practices guides immunization practices, including influenza vaccination, with recommendations revised on an annual basis. For the 2006-2007 flu season, the Advisory Committee on Immunization Practices recommendations for influenza vaccination began including healthy children aged 24-59 months (two to four years), a shift that added 10.6 million children to the target group. ${ }^{3}$

Canada has a parallel federal organization, the National Advisory Committee on Immunization, which is responsible for guiding the use of vaccines. Recommendations made by the National Advisory Committee on Immunization and the Advisory Committee on Immunization Practices around seasonal influenza vaccination was concordant until the 2006-2007 season. Starting in the 2010-2011 season, the National Advisory Committee on Immunization has further expanded its recommendations to additional pediatric age groups by including two- to four-year-olds for targeted seasonal influenza vaccination.

We took advantage of this divergence in policy between two neighboring countries with similar annual seasonal influenza epidemics to try to understand the effects of the policy change in the United States to expand influenza vaccination coverage to other pediatric populations.

\section{Methods}

We analyzed emergency department (ED) data captured by Automated Epidemiologic Geotemporal Integrated Surveillance System (AEGIS), the population health monitoring system for the Massachusetts Department of Public Health (South Street, MA, USA), from Children's Hospital Boston (Boston, MA, USA) for the 2000-2001 to 2008-2009 influenza seasons. Comparable data was extracted from the electronic records of Montreal Children's Hospital (Montreal, Quebec, Canada) to make an international comparison of rates of ED visits for influenza-like illness (ILI) following the United States vaccination policy change. Adjusting for virological factors that impact variation in influenza epidemic intensity, seasonal trends, and all-cause ED utilization, we looked for early evidence of an effect of this policy change by evaluating the post-policy change response of ILI-related ED visits to Children's Hospital Boston, using Montreal Children's Hospital as a control, for four different pediatric age groups.

\section{Results}

We observed a $34 \%$ decline in the rate of ILI-related ED visits among two- to four-year-olds for Children's Hospital Boston relative to Montreal Children's Hospital following the 2006 policy change (RR ratio 0.66 ; 95\% confidence interval: 0.59-0.75) (Table 1). We also observed smaller, marginally significant declines of $11-17 \%$ for the other age groups studied (Table 1).

\section{Conclusions}

Preschool-aged children have been identified as important to the household and community spread of influenza, due in

Table 1 Post-/pre-immunization policy change ratios of ILI-related ED visits to Children's Hospital Boston relative to Montreal Children's Hospital for four different pediatric age groups

\begin{tabular}{lcc}
\hline Age group & Adjusted rate ratio $(95 \% \mathrm{Cl})$ & $\mathrm{P}$ \\
\hline $0-1$ & $0.89(0.81,0.99)$ & 0.03 \\
$2-4$ & $0.66(0.59,0.75)$ & $<0.001$ \\
$5-9$ & $0.83(0.70,0.98)$ & 0.03 \\
$10-18$ & $0.83(0.70,0.98)$ & 0.03 \\
\hline
\end{tabular}

This is an Open Access article distributed under the terms of the Creative Commons Attribution licence (http://creativecommons.org/licenses/by/2.5) OPEN - ACCESS which permits unrestricted use, distribution, and reproduction in any medium, provided the original work is properly cited. 
part to infection rates averaging $25-43 \%$ per season, higher than any other age group. ${ }^{4}$ We observed a significant decline in ILI-related ED visits by two- to four-year-old children at Children's Hospital Boston relative to a control hospital, Montreal Children's Hospital, following the 2006-2007 divergence in pediatric influenza immunization policy. Interestingly, we also saw smaller decline in rates among other pediatric age groups, a finding that is consistent with previous evidence that vaccinating preschool-aged children also reduces population-wide morbidity due to influenza. ${ }^{5}$

\section{Acknowledgements}

This paper was presented as an oral presentation at the 2010 International Society for Disease Surveillance Conference, held in Park City, UT, USA, on 1-2 December 2010.

\section{References}

1 Henrickson KJ, Hoover S, Kehl KS, Hua W. National disease burden of respiratory viruses detected in children by polymerase chain reaction. Pediatr Infect Dis J 2004;23:S11-18.

2 Thompson WW, Shay DK, Weintraub E, Brammer L, Bridges CB, Cox NJ, et al. Influenza-associated hospitalizations in the United States. JAMA 2004;292:1333-40.

3 Advisory Committee on Immunization Practices Smith NM, Bresee JS, Shay DK, Uyeki TM, Cox NJ, Strikas RA. Prevention and Control of Influenza: recommendations of the Advisory Committee on Immunization Practices (ACIP). MMWR Recomm Rep 2006;55:1-42.

4 Fox JP, Cooney MK, Hall CE, Foy HM. Influenza virus infections in Seattle families, 1975-1979. II. Pattern of infection in invaded households and relation of age and prior antibody to occurrence of infection and related illness. Am J Epidemiol 1982;116:228-42.

5 Brownstein JS, Kleinman KP, Mandl KD. Identifying pediatric age groups for influenza vaccination using a real-time regional surveillance system. Am J Epidemiol 2005;162:686-93. 


\title{
Role of continuous monitoring on performance of surveillance systems in military populations in resource-limited settings
}

\author{
RA Hora ${ }^{1}$, DM Vera ${ }^{1}$, ZJ Silvera ${ }^{2}$, and JM Montgomery ${ }^{1}$ \\ ${ }^{1}$ US Naval Medical Research Center Detachment in Lima, Callao, Peru; and ${ }^{2}$ Directorate of Health, Peru Air Force, Lima, Peru \\ E-mail: ricardo.hora@med.navy.mil
}

\section{Objective}

The objective of this paper is to describe the effect of close monitoring on performance of the electronic disease surveillance system of the Peru Air Force.

\section{Introduction}

Timeliness of information has a key role in disease reporting, and may be easily impaired by several factors affecting data entry and utilization. ${ }^{1}$ Regarding data entry, previous studies have shown that monitoring strategies, such as telephone reminders and supervision visits ensure reporting timeliness. $^{2}$ Likewise, limited reporting infrastructure may prevent adequate reporting and effective data utilization. ${ }^{3,4}$ The Peruvian Air Force, in collaboration with the US Naval Medical Research Center Detachment in Lima, Peru, implemented in 2009 an electronic disease surveillance system with the objective of establishing near real-time baseline estimates of disease trends, and detecting disease outbreaks in a timely manner. This system has proven to perform well, although reporting sites vary in their reporting infrastructure. Therefore, we attempted to test the effect of a lack of monitoring on the performance of reporting sites, and explore the influence of other factors potentially affecting timeliness.

\section{Methods}

We analyzed the reporting performance of participating units at each epidemiological week (EW) from January to August 2010. We selected a six-week period when monitoring was to be voluntarily withheld for the purpose of our study objectives. Monitoring was resumed fully after six weeks. We compared the report-on-time rates of this six-week period of no reporting with those of the previous six-week period during which monitoring was conducted normally. Additionally, we contacted each key personnel at participating sites and asked them to complete an on-line anonymous questionnaire (www.surveygizmo.com) to explore other factors affecting performance, such as end-user's perception of the system, access to reporting media, and overall workload at establishments.

\section{Results}

From January to August 2010, all 31 active sites participated in the study. The report-on-time rate dropped from $100 \%$ (EW 17-22) to 42\% (EW 23-29) (Mann-Whitney, $P<0.01$ ). (Figure 1) Our exploratory analysis of other factors affecting timeliness showed that of the $37(100 \%)$ participants who completed the questionnaire, $30(81 \%)$ were female. The median of age was 35 years (range, 22-55). Most participants had less than five years of service $(n=14,38 \%)$; and $65 \%$ of participants were nurses and/or technicians. The overall preferred medium of reporting was the internet $(65 \%)$, although access to telephone was greater than access to the internet (60 versus 40\%, respectively). The majority of participants showed satisfaction with being part of the system (95\%), and considered reporting to be as important as their normal routine duties within the site (95\%). In addition, $84 \%$ of participants considered that reporting did not prevent them from carrying out their normal duties.

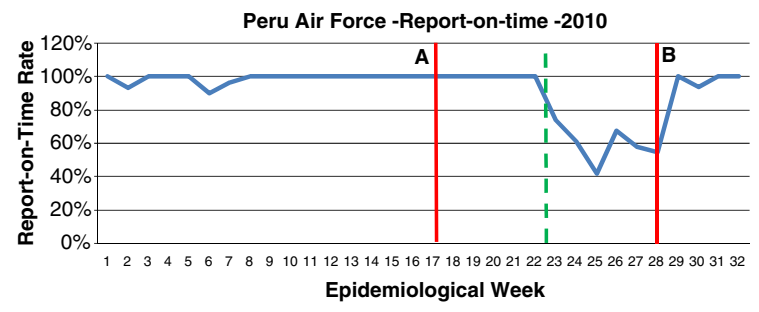

Figure 1 ROT rates during 2010. Red lines, A and B, point at the start and end dates of the study period, respectively. The green dotted line indicates the start of the six-week period at which monitoring was withheld. This is an Open Access article distributed under the terms of the Creative Commons Attribution licence (http://creativecommons.org/licenses/by/2.5)
which permits unrestricted use, distribution, and reproduction in any medium, provided the original work is properly cited. 


\section{Conclusions}

Despite availability of reporting infrastructure, and high acceptability of the system among stakeholders, the need for monitoring of reporting activities remains essential in ensuring timely reporting rates. Other factors possibly explaining this contradiction need to be further characterized.

\section{Acknowledgements}

This paper was presented as a poster at the 2010 International Society for Disease Surveillance Conference, held in Park City, UT, USA, on 1-2 December 2010.

\section{References}

1 Jajosky RA, Groseclose SL. Evaluation of reporting timeliness of public health surveillance systems for infectious diseases. BMC Public Health 2004;4:29.

2 Huaman MA, Araujo-Castillo RV, Soto G, Neyra JM, Quispe JA, Fernandez MF, et al. Impact of two interventions on timeliness and data quality [...]. BMC Med Inform Decis Mak 2009; 9:16.

3 Free MJ. Achieving appropriate design and widespread use of health care technologies in the developing world [...]. International Journal of Gynecology \& Obstetrics 2004;85 (Supplement 1): S3-13. 4 WHO. A safer future: global public health security in the 21st century. In: Hopkins D (ed). The World Health Report 2007. World Health Organization: Geneva, Switzerland, 2007. 


\title{
BioSense program redesign
}

\author{
TA Kass-Hout ${ }^{1}$, BL Massoudi ${ }^{2}$, L Rojas-Smith², SC Kaydos-Daniels², JS Brownstein ${ }^{3}$, DL Buckeridge ${ }^{4}$, and J Buehler ${ }^{1}$ \\ ${ }^{1}$ Centers for Disease Control and Prevention (CDC), Atlanta, GA, USA; ${ }^{2}$ RTI International, Durham, NC, USA; ${ }^{3}$ Epidemico, Boston, MA, USA; \\ and ${ }^{4}$ Department of Epidemiology and Biostatistics, McGill University, Montreal, Quebec, Canada \\ E-mail: tik2@cdc.gov
}

\section{Objective}

The objective of this study is that the Centers for Disease Control and Prevention (CDC) will update the International Society for Disease Surveillance community on the latest activities for the BioSense program redesign (Centers for Disease Control and Prevention, USA). ${ }^{1}$

\section{Introduction}

The BioSense program's mission is to support and improve public health surveillance infrastructure and human capacity required to monitor (with minimal lag) critical population health indicators of the scope and severity of acute health threats to the public health; and support national, state, and local responses to those threats. This mission is consistent with the 2006 Pandemic All Hazards Preparedness Act (PAHPA), and 2007 Homeland Security Presidential Directive (HSPD-21), both of which call for regional and nationwide public health situational awareness, through an interoperable network of systems, built on existing state and local situational awareness capability.

\section{Methods}

With input and guidance from our local, state, and federal surveillance partners, the new Office of Surveillance, Epidemiology and Laboratory Services (OSELS/CDC) is redesigning the BioSense program. The goal of the redesign is a new BioSense that coordinates and links existing surveillance systems to enable rapid and enhanced interchange of information.

\section{Results}

BioSense retains its original purpose of early event (or threat) detection and characterization but will expand its utility for: (1) raising public health situation awareness, (2) improving routine public health practice, (3) improving health outcomes and public health, and (4) monitoring health care quality. BioSense, as an all-hazards and timely electronic surveillance system, will provide a regional (that is, multistate) and national view of multiple health outcomes and syndromes. By integrating local and state-level data into a cohesive 'picture,' BioSense will improve its utility for state and local users.

The user requirements gathering process identified gaps in biosurveillance practices and systems that BioSense can directly address in the redesign, which will result in more effective and timely public health surveillance at the local, state, and national levels (Figure 1).

\section{Conclusions}

Activities for the BioSense program redesign process include: (1) an assessment of the business and work flow needs at the local, state, and federal levels; (2) creation and support of existing communities of practice, (3) update and realignment of the BioSense Strategic Plan to complement and

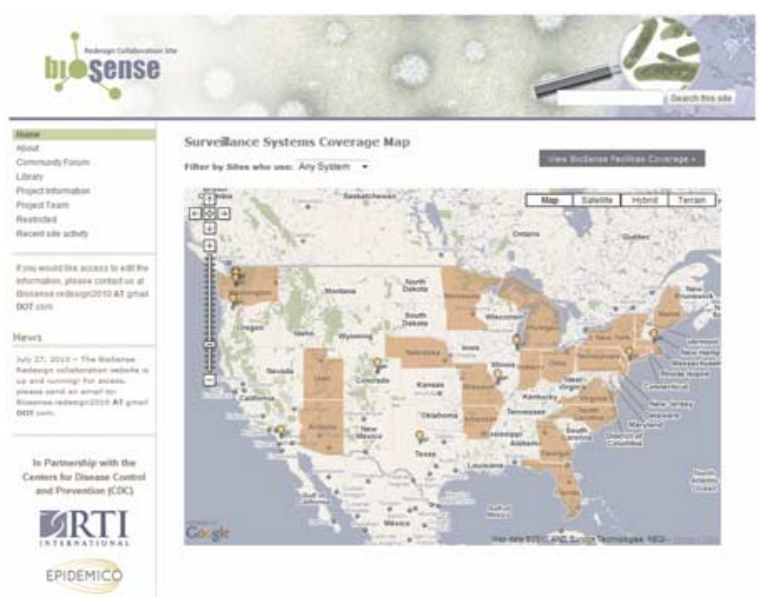

Figure 1 The BioSense Redesign Collaboration Site (https://sites.google. com/site/biosenseredesign).

open 2 Access This is an Open Access article distributed under the terms of the Creative Commons Attribution licence (http://creativecommons.org/licenses/by/2.5) OPEN - ACCESS which permits unrestricted use, distribution, and reproduction in any medium, provided the original work is properly cited. 
strengthen existing surveillance systems and meet its enhanced and broadened mission; and (4) establishment of a governance structure that will allow public health stakeholders to drive the new direction for BioSense. The new charter and governance structure is supported by a joint steering committee (or the Technical Expert Panel) with a balanced representation of local and state stakeholders and CDC. Under this new governance structure, CDC and its partners will work collaboratively to resolve problems and establish design requirements as set forth by the public health community.

\section{Acknowledgements}

This paper was presented as a poster at the 2010 International Society for Disease Surveillance Conference, held in Park City, UT, USA, on 1-2 December 2010.

\section{Reference}

1 Kass-Hout TA. BioSense Program: Going Forward. [Internet] Eighth Annual International Society for Disease Surveillance (ISDS) Conference, Miami, FL, USA, 2009. Available from: http://syndromic.org/ conference/2009/presentations/plenaries/KassHout.pdf. 


\title{
The utility of inpatient data obtained from regional health information organizations for pneumonia and influenza surveillance
}

\author{
G Johnson ${ }^{1}$, X Jin ${ }^{2}$, J-H Chen ${ }^{2}$, H-G Chang ${ }^{2}$, and D Morse ${ }^{1}$ \\ ${ }^{1}$ Office of Science, New York State Department of Health, Albany, NY, USA; and ${ }^{2}$ Division of Epidemiology, New York State Department of \\ Health, Albany, NY, USA \\ E-mail: gsb03@health.state.ny.us
}

\section{Objective}

The objective of this paper is to assess the potential utility of inpatient minimum biosurveillance data set data obtained from Regional Health Information Organizations (RHIOs) for pneumonia and influenza (P and I) surveillance.

\section{Introduction}

Hospital discharge data received by public health agencies has a reporting lag time of greater than six months. This data is often used retrospectively to conduct surveillance to assess severity of illness and outcome, and for evaluating performance of public health surveillance systems.

With the emergence of Health Information Exchanges and RHIOs, inpatient data can be available to public health in near real-time. However, there currently are no established public health practices or information systems for conducting routine surveillance in the inpatient setting.

Through a contract with the Centers for Disease Control and Prevention, New York State Department of Health initiated the development of a statewide public-health Health Information Exchanges with New York RHIOs. Daily minimum biosurveillance data set data-exchange implementation, and evaluation efforts were focused on one RHIO (RHIO A) and one participating hospital system composed of five acute-care facilities.

\section{Methods}

Inpatient data for visit dates between 1 June and 29 October 2009 were selected for analysis. The lag times between the availability of patient diagnosis data at RHIO A and the corresponding admission and discharge dates were calculated. Data obtained from RHIO A were compared with electronic hospital discharge data Statewide Planning and Research Cooperative System (SPARCS) admission diagnosis, primary discharge diagnosis, and 14 secondary diagnoses to assess the completeness and concordance of RHIO A data.
Patients were matched by facility, date of visit, gender, month and year of birth, and zip code of residence. To evaluate the utility of daily RHIO A data for inpatient P and I surveillance, text and International Classification of Diseases, Ninth Revision (ICD-9) code filters for P and I were applied to RHIO A diagnosis and reason-for-admission fields and compared with an ICD-9 code filter applied to the SPARCS diagnoses.

\section{Results}

Reason for visit was $12 \%$ complete within one day, $68 \%$ within seven days, and $98 \%$ within 30 days of admission. Diagnosis was $15 \%$ complete within one day, $73 \%$ within seven days, and $96 \%$ within 30 days of discharge.

There were 26,514 matched patients, who accounted for $99 \%$ of the SPARCS patients and $94 \%$ of RHIO A patients. Diagnosis code was present for 24,897 (94\%) RHIO A patients. Of the remaining 1617 patients, 1536 had at least one RHIO A free-text diagnosis and 81 had no RHIO A diagnosis. Unfiltered RHIO A-ICD-9 diagnosis was an exact match to one or more SPARCS diagnoses for 23,380 (94\%) patients.

When the $\mathrm{P}$ and I filters were applied to the diagnoses fields, a total of $1685(6.4 \%)$ patients met the criteria. Of those, 1601 (95\%) had a RHIO A diagnosis code that matched at least one SPARCS-ICD-9 code, 29 (1.7\%) had an uncoded RHIO A diagnosis that met the text filter criteria and at least one SPARCS diagnosis that met the ICD-9 filter criteria, 55 (3.4\%) had non-matching RHIO A and SPARCS diagnoses.

When the P and I filters were applied to RHIO A reason-foradmission, $214(0.8 \%)$ met the criteria. A total of 200 (93.5\%) patients matched at least one SPARCS-ICD-9 filtered diagnosis. Fourteen $(6.5 \%)$ patients did not have a matching ICD-9 filtered SPARCS diagnosis.

This is an Open Access article distributed under the terms of the Creative Commons Attribution licence (http://creativecommons.org/licenses/by/2.5) OPEN - ACCESS which permits unrestricted use, distribution, and reproduction in any medium, provided the original work is properly cited. 


\section{Conclusions}

Data for almost all patients reported through SPARCS was available through RHIO A and in a much more timely fashion. Both P and I filtered and unfiltered coded RHIO A diagnosis and SPARCS-ICD-9 diagnosis data matched relatively well. Adding P and I text filters to RHIO A diagnosis did not significantly improve pneumonia or influenza case ascertainment. It was not possible to assess the validity of the non-matching diagnoses in any data set. The number of patients meeting the filter criteria in was significantly lower when using RHIO A reason-for-admission compared with

using RHIO A diagnosis data. Additional analysis is underway to assess the timeliness and concordance of $\mathrm{P}$ and I outcome. Inpatient data received through RHIOs could be a valuable resource to conduct $P$ and I surveillance in the inpatient setting.

\section{Acknowledgements}

This paper was presented as an oral presentation at the 2010 International Society for Disease Surveillance Conference, held in Park City, UT, USA, on 1-2 December 2010. 


\title{
Florida's ESSENCE system - from syndromic surveillance to routine epidemiologic analysis across syndromic and non-syndromic data sources
}

\author{
A Kite-Powell ${ }^{1}$, J Hamilton ${ }^{1}$, R Wojcik ${ }^{2}$, W Loschen ${ }^{2}$, and R Hopkins ${ }^{1}$ \\ ${ }^{1}$ Florida Department of Health, Bureau of Epidemiology, Tallahassee, FL, USA; and ${ }^{2}$ Johns Hopkins University Applied Physics Laboratory, \\ Columbia, MD, USA \\ E-mail: Aaron_Kite-Powell@doh.state.fl.us
}

\begin{abstract}
Objective
Use of the Electronic Surveillance System for the Early Notification of Community-based Epidemics (ESSENCE) in Florida has evolved from early event detection based on emergency department (ED) chief complaints to routine descriptive epidemiologic analysis, data visualization, and reporting across four different data sources, using and building on tools originally developed for syndromic surveillance.
\end{abstract}

\section{Introduction}

Federal laws and national directives have focused attention on the development of more robust biosurveillance systems intended to detect events of public health interest in a timelier manner. Presidential Decision Directive 21 calls for integrated biosurveillance data, enhanced clinician awareness, and an epidemiologic surveillance system with sufficient flexibility to tailor analyses to new syndromes and emerging diseases. In 2007, a statewide syndromic surveillance system (ESSENCE) was implemented and hospitals were recruited to participate. Experience with ESSENCE in the context of the ED data analysis, visualization, and reporting prompted the exploration of integrating new data sources into ESSENCE and new analyses specific to these new data. The purpose of the ESSENCE system is now to provide an intuitive environment for state and local epidemiologists to conduct routine descriptive epidemiologic analysis, to monitor morbidity and mortality trends over time and space and across multiple data sources, thereby providing information that can assist with making decisions on how to improve population health.

\section{Methods}

The Florida ESSENCE system is a multi-tiered, automated, and secured (https) web-based application. Microsoft SQL
(One Microsoft Way, Redmond, WA, USA) databases are used in data ingestion, statistical analysis, and web databases. System development is primarily in Java (Oracle Corporation, Redwood Shores, CA, USA). It can be accessed via username and password by state-level epidemiologists, county health department epidemiologists, and staff at hospitals that participate. Enhancements to the system are developed through close collaboration with the Johns Hopkins University Applied Physics Laboratory, paying close attention to feedback from state and local epidemiologists in Florida.

\section{Results}

The system in Florida now includes four different data sources, each with its own module: (1) ED record data from 145 hospitals (updated once daily); (2) Merlin reportable disease case data from the Merlin system (updated once an hour); (3) Florida Poison Information Network consultation data (updated every $20 \mathrm{~min}$ ); and (4) Florida Office of Vital Statistics death data (updated once daily). All records have individual identifiers removed.

Users can produce time-series graphs, maps, and tables by a number of possible stratification variables per data source. Several data sources can be combined in the same view. Time-series analysis can be conducted on daily, weekly, monthly, quarterly, and annual time resolutions. Statewide pneumonia and influenza mortality from the Office of Vital Statistics death data feed to ESSENCE is displayed as weekly counts and a weekly proportional mortality, and uses a Serfling regression model for analysis. Death certificates can be analyzed on the basis of analysis of literal text with a five-week delay and on assigned International Classification of Diseases and Related Health Problems 10th revision (ICD-10) codes with a 12-week delay. ED data are analyzed by sub-day time intervals to

This is an Open Access article distributed under the terms of the Creative Commons Attribution licence (http://creativecommons.org/licenses/by/2.5) OPEN ACCESS which permits unrestricted use, distribution, and reproduction in any medium, provided the original work is properly cited. 
identify clusters by the time patients are registered at the ED by hospital and sub-syndrome category. The Merlin reportable disease data are now processed through ESSENCE's spatial scan statistic to assist with detecting spatial clusters of public health significance.

\section{Conclusions}

A number of barriers often exist for users attempting to access public health data. Data are sometimes siloed within organizations, and often very specialized skill sets are needed to manipulate and analyze data in statistical and mapping software programs. The integration of four data sources into the Florida ESSENCE system creates one location, where users can go to access data and create epidemiologic reports and 'data dashboards' that describe the morbidity and mortality for a given region in Florida. This functionality has allowed for more timely and routine analysis of data that was previously not available, or required significant effort to access, and has helped close the public health surveillance loop with our partners in the clinical community.

\section{Acknowledgements}

This paper was presented as an oral presentation at the 2010 International Society for Disease Surveillance Conference, held in Park City, UT, USA, on 1-2 December 2010. 


\title{
Comparison of Distribute and ILINet for national influenza surveillance
}

\author{
K Kniss $^{1,2}$, S Benoit ${ }^{2}$, A Mclntyre ${ }^{2}$, D Buckeridge ${ }^{3}$, J Brownstein ${ }^{3}$, L Brammer ${ }^{2}$, and L Finelli ${ }^{2}$ \\ ${ }^{1}$ Atlanta Research and Education Foundation, Atlanta, GA, USA; ${ }^{2}$ Centers for Disease Control and Prevention, Influenza Division, Atlanta, \\ GA, USA; and ${ }^{3}$ International Society for Disease Surveillance, Boston, MA, USA \\ E-mail: krk9@cdc.gov
}

\section{Objective}

To compare ILI data reported to the Distribute surveillance project to data from an existing influenza surveillance system, the US Outpatient Influenza-like Illness Network (ILINet).

\section{Introduction}

During the spring of 2009, a public health emergency was declared in response to the emergence of the 2009 Influenza A (H1N1) virus. Owing to the response, timely data were needed to improve situational awareness and to inform public health officials. Traditional influenza surveillance is time-consuming and resource intensive, and electronic data sources are often more timely and resource saving. Collaboration began between the Centers for Disease Control and Prevention (CDC), the International Society for Disease Surveillance (ISDS), and the Public Health Informatics Institute (PHII) to expand syndromic Emergency Department (ED) surveillance through the Distribute project.

Distribute collects aggregate, daily or weekly reports of influenza-like illness (ILI) and total patient visits to EDs from participating health jurisdictions, stratified by age group and other variables. Additional variables included the three digit zip code of the patient's residence as well as the disposition and temperature, however not all jurisdictions collect these variables. ${ }^{1}$ Distribute data are typically extracted from EDbased electronic health data systems. The ILI definition is determined by the participating jurisdiction that can be a city, county, or state. At the time of analysis, the network consisted of 33 jurisdictions.

Because ILI data reported to Distribute had not been systematically compared with data reported through other surveillance systems, CDC planned an evaluation of the Distribute data, which included a comparison to ILINet.

ILINet is a collaborative effort between the CDC, local and state health departments and primary health care providers. The network currently consists of approximately 3000 healthcare providers in all 50 states, Chicago, the District of Columbia, New York City, and the US Virgin Islands. Enrolled providers send CDC weekly reports via internet or fax that consist of the total number of patients seen for any reason and the number of those patients with ILI by age group. ILI is defined as fever (temperature of $\geqslant 100^{\circ} \mathrm{F}$ $\left(37.8^{\circ} \mathrm{C}\right)$ ) and a cough and/or sore throat in the absence of a known cause other than influenza. ${ }^{2}$

\section{Methods}

Data from Distribute and ILINet were obtained from 2 September 2007 through 31 October 2009 for all sites where data were available. The weekly percent of visits because of ILI were calculated for each system. For statebased Distribute jurisdictions comparisons were made using ILINet data from the same state. For local jurisdictions, a geographic area was defined using the appropriate city or county boundaries. ILINet facilities that fell into the defined boundaries were used for comparison. Comparisons were made using correlation coefficients as well as visually.

\section{Results}

For state-based jurisdictions the correlation coefficients ranged from 0.64 to 0.96 , with a mean of 0.83 . For local jurisdictions, the correlation coefficients ranged from 0.38 to 0.91 with a mean of 0.76 . For all state jurisdictions and all but one local jurisdiction, peaks in ILI tracked similarly in both systems For areas known to have the same EDs contributing data to both systems, ED data were removed from the ILINet data, and comparisons were made again. Correlations among these sites changed very little when the ED data were removed from the ILINet data.

\section{Conclusions}

Overall, the correlation between ILI data reported to the two systems was high. These results show that similar ILI data can be obtained using either electronic ED-based syndromic surveillance or traditional, provider-

This is an Open Access article distributed under the terms of the Creative Commons Attribution licence (http://creativecommons.org/licenses/by/2.5) OPEN - ACCESS which permits unrestricted use, distribution, and reproduction in any medium, provided the original work is properly cited. 
based syndromic surveillance, and could be potentially useful in tracking and describing influenza activity at the national level. Differences in data collected through the two systems could be because of differences in the populations under surveillance, or potentially because of differences in illness seen in emergency departments compared with those seen in traditional primary care. Periodic evaluation of these systems should be conducted. Efforts to expand the Distribute network to a national network should be made.

\section{Acknowledgements}

This paper was presented as a poster at the 2010 International Society for Disease Surveillance Conference, held in Park City, UT, USA, on 1-2 December 2010.

\section{References}

1 Distribute background at: http://www.isdsdistribute.org/faq.php.

2 Influenza Surveillance Background: http://www.cdc.gov/flu/ weekly/fluactivity.htm. 


\title{
Emergency department diagnosis code data for surveillance of vaccine adverse events: comparison with the national vaccine adverse event reporting system
}

\author{
E Lamb¹, H Vaughan-Batten', NJM Dailey ${ }^{1,2}$, J-M Maillard ${ }^{1}$, L Johns ${ }^{1}$, AT Fleischauer ${ }^{1,2}$, Z Moore ${ }^{1}$, and M Davies ${ }^{1}$ \\ ${ }^{1}$ Division of Public Health, North Carolina DHHS, Raleigh, NC, USA; and ${ }^{2}$ Center for Disease Control and Prevention, Atlanta, GA, USA \\ E-mail: emilie.lamb@dhhs.nc.gov
}

\begin{abstract}
Objective
The objectives of this study were: (1) to compare trends in vaccine adverse events identified through emergency department (ED) diagnosis codes and reports from the Vaccine Adverse Event Reporting System (VAERS), and (2) to determine whether $2009 \mathrm{H} 1 \mathrm{~N} 1$ vaccine adverse events identified through VAERS could also be identified using ED diagnosis codes.
\end{abstract}

\section{Introduction}

Nationally, vaccine safety is monitored through several systems including Vaccine Adverse Event Reporting System (VAERS), a passive reporting system designed to detect potential vaccine safety concerns. ${ }^{1}$ Healthcare providers are encouraged to report adverse events after vaccination to VAERS, whether or not they believe that the vaccine caused the adverse event. ${ }^{1}$ The 2009 Pandemic H1N1 influenza vaccine became available in the United States in October 2009. By January 2010, Center for Disease Control and Prevention (Atlanta, GA, USA) estimated that 61 million persons across the United States had received the vaccine. ${ }^{2}$ As of January 2010, an estimated 28\% of the North Carolina population greater than or equal to six months of age had been vaccinated against $2009 \mathrm{H} 1 \mathrm{~N} 1 .^{3}$

\section{Methods}

Vaccine adverse events among North Carolina residents with symptom onset during 1 January 2008-31 December 2009 were identified using VAERS reports and emergency department (ED) diagnosis codes. The ED diagnosis codes for bacterial and other vaccines causing adverse effects in therapeutic use (ICD-9-CM codes E948-E949.9) were obtained from the North Carolina Disease Event Tracking and Epidemiologic Collection Tool (NC DETECT) that collects data from $99 \%$ of EDs statewide. ${ }^{4}$ We used Pearson's correlation coefficient to compare trends in the weekly number of VAERS vaccine adverse event reports with events identified using ED diagnosis codes. We identified adverse events from 2009 H1N1 vaccination during 1 October 200931 January 2010 using VAERS reports, and attempted to match reports that indicated that the patient had visited either an ED, or physician's office to ED visits with diagnosis codes possibly related to influenza vaccination (E949.6, E949.7, and E949.9). Events were matched by age, sex, date of birth, county of residence, and vaccine administration.

\section{Results}

We identified 1793 vaccine adverse event reports using VAERS and 782 events through ED diagnosis codes among North Carolina residents with symptom onset or visit date during 1 January 2008-31 December 2009. We detected a moderate temporal correlation between vaccine adverse events identified from ED diagnosis codes and VAERS reports $(r=0.47413)$ Figure 1 . Of two hundred and seventy two 2009 $\mathrm{H} 1 \mathrm{~N} 1$ vaccine adverse event reports sent to VAERS regarding North Carolina residents with onset during 1 October 2009-31 January 2010, 100 indicated that the patient visited the ED or a physician's office. Of these, only $8 \%$ could be matched to cases identified by ED diagnosis codes.

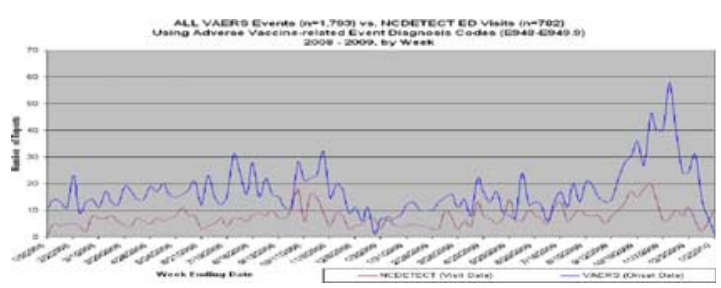

Figure 1 Weekly number of VAERS reports and NC DETECT-ED visits with symptom onset or visit dates during 1 January 2008-31 December 2009.

This is an Open Access article distributed under the terms of the Creative Commons Attribution licence (http://creativecommons.org/licenses/by/2.5) OPEN - ACCESS which permits unrestricted use, distribution, and reproduction in any medium, provided the original work is properly cited. 


\section{Conclusion}

Temporal trends in ED visits for vaccine adverse events correlate moderately well with trends in VAERS reports. However, the small number of $2009 \mathrm{H} 1 \mathrm{~N} 1$ vaccine adverse event, VAERS reports, identified by ED diagnosis codes indicate that different patient populations or types of events are captured by these systems. Further prospective study is required to determine if $\mathrm{ED}$ diagnosis code surveillance could prove useful for monitoring trends in vaccine adverse events.

\section{Acknowledgements}

This paper was presented as an oral presentation at the 2010 International Society for Disease Surveillance
Conference, held in Park City, UT, USA, on 1-2 December 2010.

\section{References}

1 Varricchio F, Iskander J, Destefano F, Ball R, Pless R, Braun MM, et al. Understanding vaccine safety information from the Vaccine Adverse Event Reporting System. Pediatr Infect Dis J 2004;23(4): 287-9.

2 CDC. Interim Results: Influenza A (H1N1) 2009 Monovalent Vaccination Coverage-United States, October-December 2009. MMWR 2010;59 (No. 2): 44-8.

3 CDC. Interim Results: State-Specific Influenza A (H1N1) 2009 Monovalent Vaccination Coverage - United States, October 2009January 2010. MMWR 2010;59 (No. 12): 363-638.

4 Waller A, Hakenewerth A, Tintinalli J, Ising A. North Carolina Emergency Department Data, January 1, 2007-December 31, 2007. N C Med J 2010;71(1): 15-25. 


\title{
Meaningful use and public health surveillance: to travel fast or far?
}

\author{
LA Lenert
}

Department of Medicine and Biomedical Informatics, University of Utah School of Medicine, Salt Lake City, UT, USA E-mail: leslie.lenert@hsc.utah.edu

\section{Objective}

The objective of this paper is to review the limitations of current approaches to linkage of public health through meaningful use reporting requirements and to explore alternatives based on integration of public health data reporting requirements, with clinical quality improvement reporting requirements.

\section{Introduction}

There is an ancient African proverb that states, 'If you want to travel fast, travel alone-if you want to travel far, travel together.' This paper examines the issue of whether public health can and should 'go it alone' in efforts for creating linkages between clinical care systems and the public health sector, as part of meaningful use requirements. 'Going it alone' in this circumstances refers to whether public health should seek to require data flows, through meaningful use requirements, that meet its work flow needs but do not add value to clinical work flows. An alternative would be to look for synergies between public health goals and the goals of the clinical care system, which public health could exploit to achieve its ends through collaborative means.

\section{Methods}

Efforts to create meaningful use requirements are reviewed through the lens of social competition between public health interests seeking more data and more access, healthcare providers seeking to minimize costs and to prevent exposure from loss of confidentiality, and federal regulators seeking workable accommodations that move the state of the programs forward, balancing the needs of society.

\section{Results}

Although numerous proposals for submission of data to public health entities were discussed during a series of meetings held by the National Committee of Vital Statistics in Spring of 2009, only two types of data sharing were specified under the final rule for healthcare providers-sharing with immunization registries or submission of syndromic data to health departments, where such capacity exists. Rules for hospitals allow a choice between any of the three tasks: the former two, plus the option of submitting reportable laboratory results to public agencies. The result is an unfavorable one for public health agencies. Agencies need to provide the infrastructure to support all three types of submission, but providers may chose the type of data most convenient for them to submit. Further, because data types submitted by providers are self-selected, the value of the data of public health is diminished. Although the rule allows states to 'require more' data submission, incentives will be paid to all providers who submit one type of data, regardless of any additional requirements (that is, no enforcement.) The conclusion: public health lost this first round of negotiations on integration. It has the most requirements, received only minimal funding from HITECH to support its infrastructure needs to receive data, and has only limited ability to influence the choice that providers make in the type of data they submit through state legislation.

Consider, in contrast, how efforts to enhance population health through quality reporting fared. By 2112, the regulations will require denominator-based reporting on a core set of quality measures pertaining to blood pressure control, smoking cessation, and obesity (or on alternate core measures that include childhood immunization rates). There is also an additional list of 38 other potential quality measures that can be selected for reporting-these are substantiative requirements and everyone must participate.

What if public health chose to align its push for data from meaningful use with these quality measures? For example, smoking cessation quality indicators could be transformed into data on the prevalence of smoking in the practice and on the incidence of cessation interventions? Might not this data, which would use the same mechanisms and infrastructure used to produce quality reporting, be as valuable for public health, from a policy perspective, as data feeds to traditional surveillance systems? The value of the data could

This is an Open Access article distributed under the terms of the Creative Commons Attribution licence (http://creativecommons.org/licenses/by/2.5) OPEN ACCESS which permits unrestricted use, distribution, and reproduction in any medium, provided the original work is properly cited. 
be further enhanced by use of geocoding strategies, such as Geographic Interoperable Patient Summary Exchange formatting, which would allow combination of data across practices and views of the health of small areas and into health disparities.

\section{Conclusions}

In addition to the well-known (and experienced) financial limitations that public health faces, in social systems where public health interests compete with those of other sectors, there are limits on the political capital that public has as well. Applying that capital in a way that is synergistic, with other interests in the healthcare sector, may produce better long-term results than going against the interests of the sector. Given the alignment between quality of care measures and population level surveillance of chronic diseases, public health may travel farther 'together' alone in pursuit of traditional surveillance measures.

\section{Acknowledgements}

This paper was presented as an oral presentation at the 2010 International Society for Disease Surveillance Conference, held in Park City, UT, USA, on 1-2 December 2010. 


\title{
Surveillance for nationally notifiable infectious conditions using ICD-9-CM diagnosis codes in the VA ESSENCE biosurveillance system
}

\author{
C Lucero, P Schirmer, G Oda, and M Holodniy \\ Department of Veterans Affairs, Office of Public Health Surveillance and Research, Palo Alto, CA, USA \\ E-mail: cynthia.lucero@va.gov
}

\section{Objective}

To determine the utility of ICD-9-CM diagnosis codes in the Veterans Affairs (VA) Electronic Surveillance System for the Early Notification of Community-based Epidemics (ESSENCE) for detection and public health surveillance of nationally notifiable infectious conditions in veteran patients.

\section{Introduction}

ESSENCE obtains electronic data from 153 VA Medical Centers plus outpatient clinics in all 50 states, American Samoa, Guam, Philippines, Puerto Rico, and U.S. Virgin Islands. Currently, there is no centralized VA reporting requirement for nationally notifiable infectious conditions detected in VA facilities. Surveillance and reporting of cases to local public health authorities are performed manually by VA Infection Preventionists (IP) and other clinicians. In this analysis, we examined positive predictive value (PPV) of ICD-9-CM diagnosis codes in VA ESSENCE to determine the utility of this system in electronic detection of reportable conditions in VA.

\section{Methods}

VA ESSENCE analyzes ICD-9-CM diagnosis codes and demographic data from outpatient and emergency department visits at all VA facilities. ${ }^{1}$ For this review, visits with an ICD-9-CM code(s) for a reportable communicable disease during 2009 were selected. For diseases in which 10 or fewer potential cases were identified, we expanded the date range and selected all potential cases from July 2005 to May 2010. For diseases in which more than 100 potential cases were identified, a minimum of $20 \%$ of records were selected for review. Laboratory and clinical data from electronic medical records were reviewed by a VA epidemiologist for concordance and case classification based on the most recent CDC/CSTE case definitions. ${ }^{2}$ PPV for each disease was calculated as number of patients who met case definition criteria divided by total number assigned an ICD-9-CM diagnosis code for the disease.
For some diseases, numerators included both confirmed and probable/ suspected cases based on CDC definitions.

\section{Results}

Disease-specific PPV proportions ranged from 0 to $72 \%$ with the overall proportion $25.7 \%$ (224/871). Of the 30 reportable diseases reviewed thus far, 13 diseases had PPVs of $0 \%$. Of the 30 diseases, 15 had PPVs above 10\% and 4 diseases (cryptosporidiosis, listeriosis, spotted fever rickettsiosis, and salmonellosis) had PPVs greater than 50\%. Reasons that visits did not meet CDC case definitions included: (1) Patient had a history of the disease but was not acutely infected; (2) Miscoding for vaccine administration or vaccine reaction; (3) Miscoding of similar sounding disease; (4) Patient was initially diagnosed or treated at another facility, so testing results not available in VA records; (5) Diagnosis was in the differential or a clinical diagnosis only with no confirmatory testing performed; (6) Miscoding for prophylaxis given or exposure to a disease but patient was not acutely ill; (7) Miscoding for antibody titer evaluations.

\section{Conclusions}

PPVs for the majority of reportable communicable diseases captured by current VA ESSENCE were low, and therefore ESSENCE is of limited value in detecting new cases. Our results are consistent with a similar analysis performed in North Carolina. ${ }^{3}$ For a few diseases, ESSENCE had relatively high PPVs (greater than 50\%). For these diseases, VA ESSENCE may be an added tool for more timely and possibly for additional case detection beyond what is currently captured through manual surveillance. Future work will evaluate system sensitivity by comparing reportable diseases captured by VA ESSENCE with those identified by IP manual surveillance as a gold standard. Efforts to enhance PPV by combining ICD-9-CM codes with additional data elements (for example, laboratory results) are also underway. Accurate, automated, electronic reporting of reportable diseases to VA Office of Public Health will be

open 2 access This is an Open Access article distributed under the terms of the Creative Commons Attribution licence (http://creativecommons.org/licenses/by/2.5) OPEN ACCESS which permits unrestricted use, distribution, and reproduction in any medium, provided the original work is properly cited. 
important in understanding disease trends within Veteran populations, ensuring timely reporting and facilitating information sharing with local, state, and federal public health partners.

\section{Acknowledgements}

This paper was presented as an oral presentation at the 2010 International Society for Disease Surveillance Conference, held in Park City, UT, USA, on 1-2 December 2010.

\section{References}

1 Lombardo J, Burkom H, Elbert E, Magruder S, Lewis SH, Loschen W, et al. A systems overview of the Electronic Surveillance System for the Early Notification of Community-Based Epidemics. J Urban Health 2003;80 (2 Suppl 1): i32-42.

2 CDC. Nationally Notifiable Infectious Conditions, United States 2010. Available at: http://www.cdc.gov/ncphi/disss/nndss/phs/ infdis2010.htm.

3 Sickbert-Bennett EE, Bischoff T, Cleve W, Long R. Utility of ICD-9CM Diagnosis Codes for Public Health Surveillance. Advances in Disease Surveillance 2008;5:62. 


\title{
Using laboratory and medical encounter records to identify impact of prophylaxis policies on group $A$ Streptococcus in three recruit training facilities
}

\author{
TM Luse, K Shannon, and G Nowak \\ EpiData Center Division, Navy and Marine Corps Public Health Center, Portsmouth, VA, USA \\ E-mail: tina.luse@med.navy.mil
}

\section{Objective}

To compare trends of group A beta-hemolytic Streptococcus among recruits before and after changes in prophylaxis implementation using electronic laboratory and medical encounter records.

\section{Introduction}

Group A beta-hemolytic Streptococcus (GABHS) has caused outbreaks in recruit training environments, where it leads to significant morbidity and, on occasion, has been linked to deaths. ${ }^{1,2}$ Streptococcal surveillance has long been a part of military recruit public health activities. All Navy and Marine Corps training sites are required to track and record positive throat cultures and rapid tests on weekly basis. The Navy and Marine Corps have used bicillin prophylaxis as an effective control measure against GABHS outbreaks at recruit training sites. ${ }^{3}$ Though streptococcal control program policies vary by site, a minimum prophylaxis protocol is required and mass prophylax is indicated when local GABHS rates exceed a specific threshold. Before July 2007, prophylaxis upon initial entry was required between October and March, and when the local rate exceeded 10 cases per 1000 recruits. In July 2007, the Navy instituted a policy of mass prophylaxis upon initial entry throughout the year. Evaluation of GABHS cases before and after implementation of the new policy, including overall rates, identification of outbreaks, and inpatient results will help enhance the Navy's ability to evaluate threshold levels, provide systematic/standardized monitoring across the three recruit sites, and inform prophylaxis and monitoring strategies.

\section{Methods}

Positive GABHS laboratory results were identified from the Health Level 7 chemistry and microbiology databases from 1 May 2004 through 31 December 2009 for all Navy and Marine Corps recruits. Laboratory results included cultures and rapid strep tests. Positive laboratory test results were matched on specimen collection dates to inpatient and outpatient clinical encounter records using ICD-9 codes. The top diagnoses among GABHS cases were identified. Using these most frequent diagnoses associated with strep positive labs, inpatient and outpatient encounter records during the study timeframe were identified for all recruits with GABHSlike illness, regardless of laboratory test result. Weekly GABHS rates were calculated for laboratory positive cases and GABHS-like diagnosis for each recruit training site. Weekly trends were compared with prophylaxis regimens for each training site.

\section{Results}

There were 5576 laboratory-identified cases of GABHS in Navy and Marine Corps recruits between May 2004 and December 2009. Almost half of all cases $(47 \%, n=2596)$ occurred at one of the three recruit training sites. Comparisons of illness rates before and after implementation of the new policy showed a substantial decrease in rates at all three training facilities. In 2008 and 2009, rates of illness each week rarely exceeded two cases per 1000 individuals and never exceeded four cases per 1000 individuals. Large peaks in cases were identified often before 2007, but have not occurred since 2008. A total of 200,914 outpatient records were identified with GABHS-like illnesses. Close to 60 percent of all outpatient encounters occurred at one of the three training sites $(n=118,043)$. Volume of cases was substantially lower after implementation of prophylaxis policies.

\section{Conclusion}

Identifying cases of GABHS using electronic laboratory and medical encounter records can assist in determining whether prophylaxis policies are effective. Evaluation of the data has shown a substantial decrease in rates of cases among recruits after the implementation of the new policy in 2007 .

OPens This is an Open Access article distributed under the terms of the Creative Commons Attribution licence (http://creativecommons.org/licenses/by/2.5) OPEN ACCESS which permits unrestricted use, distribution, and reproduction in any medium, provided the original work is properly cited. 


\section{Acknowledgements}

This paper was presented as an oral presentation at the 2010 International Society for Disease Surveillance Conference, held in Park City, UT, USA, on 1-2 December 2010.

\section{References}

1 Crum NF, Hale BR, Judd SE, Lim ML, Wallace MR. A case series of group A Streptococcus necrotizing fasciitis among military trainees. Mil Med 2004;169:373-5.

2 Centers for Disease Control and Prevention. Outbreak of group A streptococcal pneumonia among marine corps recruits-California, November 1-December 20, 2002.

3 Russell KL. Recruit Medicine. Borden Institute, Walter Reed Medical Center: US Government Printing Office, Washington, DC, 2006. 


\title{
EpiSentry $^{\mathrm{TM}}$ : simulation-based threat identification, response management and decision support for outbreaks and pandemics
}

\author{
DW Mazeroski, and T Arthur-Hartranft
}

Lockheed Martin Corporation, IS\&GS, Lansdale, PA, USA

E-mail: david.w.mazeroski@Imco.com

\begin{abstract}
Objective
Traditional real time surveillance systems such as RODS and ESSENCE have focused on the task of threat detection; however, experience with the use of these systems in pandemic and disaster response settings suggests that a more common application is threat characterization and response management. This paper describes EpiSentry ${ }^{\mathrm{TM}}$ : a novel second generation real-time surveillance software system under development at Lockheed Martin that uses simulation to aid in threat characterization, response management and to provide decision support for disease outbreaks or bio-terror events.
\end{abstract}

\section{Introduction}

The 2009 H1N1 novel flu pandemic demonstrates how a rapidly spreading, contagious illness can affect the world's population in multiple ways including health, economics, education, transportation, and national security. Pandemic disease and the threat of bio-terrorism are prompting the need for a system that integrates disparate data, makes optimal use of the breadth of available health-related analysis and predictive models, and provides timely guidance to decision makers at multiple levels of responsibility.

\section{Methods}

Lockheed Martin Corporation is currently developing the EpiSentry $^{\mathrm{TM}}$ rapid decision environment. EpiSentry ${ }^{\mathrm{TM}}$ integrates epidemiological situational assessment and predictive analysis tools with a decision support system to provide guidance on pandemic and bio-terror investigations (Figure 1).

The project is using an agile development methodology to address the technical challenges of integration of surveillance, simulation and planning capabilities in a series of progressively more advanced software solutions. Challenges include:

- Ability to extrapolate relevant information from nontraditional data sources before data from traditional surveillance streams may even be available.

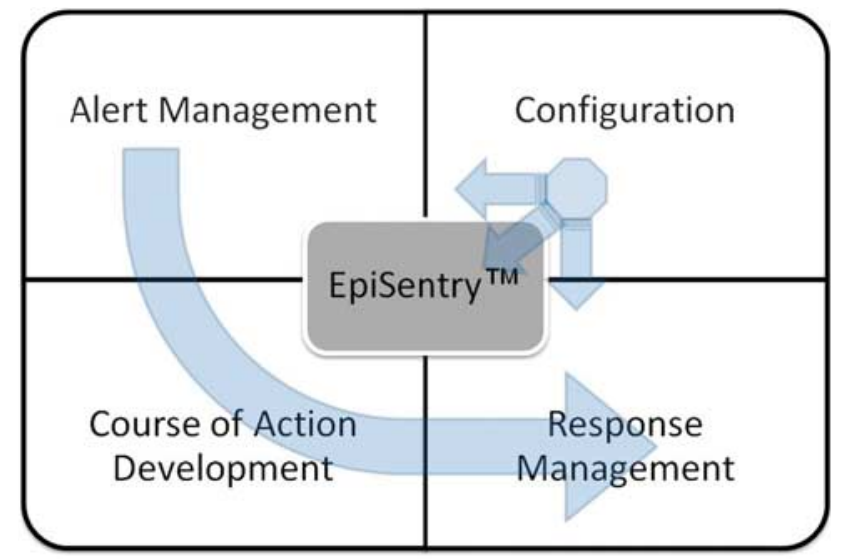

Figure 1 The EpiSentry ${ }^{\mathrm{TM}}$ framework provides an integrated configurable alert management, course of action development, and response management capability.

- Estimation (from both traditional and non-traditional surveillance data) of population-level parameters, with modeling of disease spread and impacts of countermeasures.

- Development of workflow tools for collaborative investigation and response planning.

As part of its solution, EpiSentry ${ }^{\mathrm{TM}}$ employs geospatial visualization and data fusion techniques, integrates open source monitoring and trending solutions, develops an enhanced disease mobility module, and leverages a decision analytical framework to provide the user with evidence projections of the risks and benefits of different action plans.

\section{Results}

Although still in development, we believe EpiSentry ${ }^{\mathrm{TM}}$ will significantly advance contemporary response management. A system built around EpiSentry ${ }^{\mathrm{TM}}$ will be part of a bold strategy to meet information needs for the management of This is an Open Access article distributed under the terms of the Creative Commons Attribution licence (http://creativecommons.org/licenses/by/2.5)
which permits unrestricted use, distribution, and reproduction in any medium, provided the original work is properly cited. 
outbreaks. It will provide an integrated, end-to-end, and collaborative solution that enables the use of advanced algorithms and simulation software to monitor and identify outbreaks from disparate data sources, plan intervention strategies, and monitor the effectiveness of the chosen course of action.

\section{Conclusions}

EpiSentry $^{\mathrm{TM}}$ is a new type of surveillance system that combines data analysis and outbreak modeling, and response-planning software systems. Although promising, further work is needed to define the benefits of this integrated approach.

\section{Acknowledgements}

This paper was presented as a poster at the 2010 International Society for Disease Surveillance Conference, held in Park City, UT, USA, on 1-2 December 2010. 


\title{
ABSTRACT
}

\section{Quantifying the relationship between influenza-related emergency department visits and hospital admissions in BioSense}

\author{
AF McIntyre ${ }^{1}$, L Finelli ${ }^{1}$, HS Burkom ${ }^{2}$, SR Benoit ${ }^{1}$, KL Kniss $^{1}$, and TA Kass-Hout ${ }^{1}$ \\ ${ }^{1}$ Centers for Disease Control and Prevention, Atlanta, GA, USA; and ${ }^{2}$ Johns Hopkins University Applied Physics Laboratory, Baltimore, MD, USA \\ E-mail: AMcIntyre@cdc.gov
}

\section{Objective}

The purpose of this analysis is to gain understanding of the burden of influenza in recent years through analysis of clinically rich hospital data. Patterns of visits and severity measures such as the ratio of admissions related to influenzalike illness (ILI) by age group from 2007 to 2010 are described.

\section{Introduction}

Real-time emergency department (ED) data from the BioSense surveillance program for ILI visits and ILI admissions provide valuable insight into disease severity that bridges gaps in traditional influenza surveillance systems that monitor ILI in outpatient settings and laboratory-confirmed hospitalization, but do not quantify the relationship between ILI visits and hospital admissions.

\section{Methods}

All patients with ILI presenting to EDs participating in BioSense ( $n=650$ hospitals) during September 2007 through June 2010 were included in this analysis. ILI visits were defined as encounters with an influenza diagnosis code

Ratio of Hospital Admissions to Influenza-related Emergency Department Visits 2007-2010

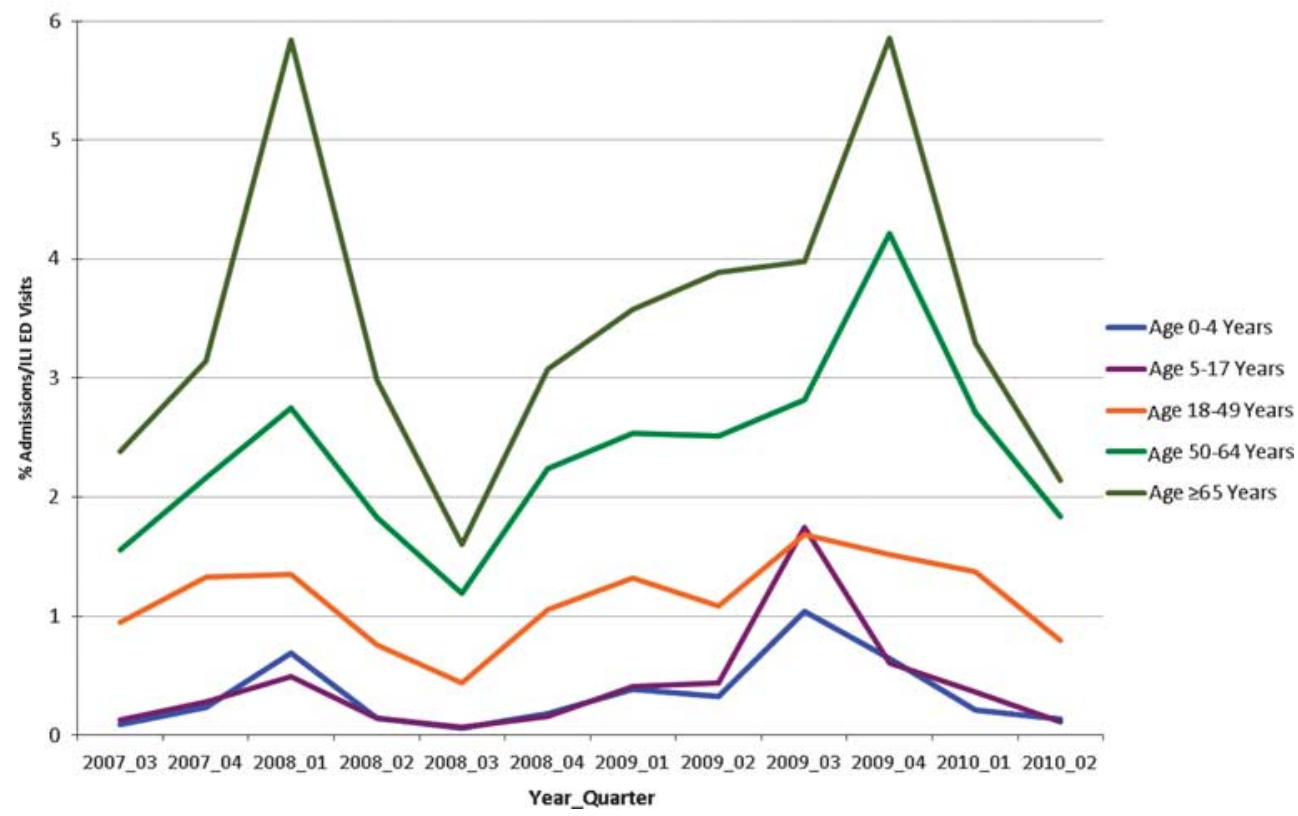

Figure 1 Ratio of hospital admissions to influenza-related emergency department visits.

open 2 Access This is an Open Access article distributed under the terms of the Creative Commons Attribution licence (http://creativecommons.org/licenses/by/2.5) OPEN - ACCESS which permits unrestricted use, distribution, and reproduction in any medium, provided the original work is properly cited. 
(ICD-9-CM codes 487 and 488) or an ILI-related chief complaint ('influenza' or fever and cough/upper respiratory infection). Age groups were defined as 0-4 years, 5-17 years, $18-49$ years, $50-64$ years, and $\geqslant 65$ years. Those with unknown age or age $>105$ years were excluded. To account for age and seasonal differences, frequencies and mean were stratified by age group and by calendar year quarter to calculate the proportion of ILI-related ED visits resulting in hospitalization. Analysis of variance was performed to assess differences in mean hospitalization encounters by age group and calendar year quarter.

\section{Results}

There were 1,925,539 patients who sought care for ILI at EDs reporting to BioSense during September 2007 through June 2010. Among those with ILI, 59,294 (3.1\%) were hospitalized. The admission to ILI ratio was similar $(P=0.58)$ by quarter with $2.92 \%$ (range $2.65-3.14 \%$ ) of visits admitted in Quarter 1, 3.00\% (range 2.79-3.32\%) in Quarter 2, 3.94\% (range 2.86-5.33\%) in Quarter 3, and 3.33\% (range 2.18$4.84 \%$ ) in Quarter 4. The mean admission to ILI ratio was highest in those aged $\geqslant 65$ years $(\mathrm{M}=3.48 \%$, s.d. $=1.31)$, followed by 50-64 years $(\mathrm{M}=2.36 \%$, s.d. $=0.77), 18-49$ years $(\mathrm{M}=1.34 \%$, s.d. $=0.36), 5-17$ years $(\mathrm{M}=0.41$, s.d. $=0.46)$, and $0-4$ years $(\mathrm{M}=0.34 \%$, s.d. $=0.30)$, although there were no significant differences by age group. By quarter and age group, the highest admissions occurred during Quarter 1 among those aged $\geqslant 65$ years $(M=4.24 \%$, s.d. $=1.40), 18-49$ years $(\mathrm{M}=1.35 \%$, s.d. $=0.03)$, and $0-4$ years $(\mathrm{M}=0.43 \%$, s.d. $=0.24)$, during Quarter 3 for 5-17 years $(M=0.64 \%$, s.d.=0.96), and during Quarter 4 for 50-64 years $(\mathrm{M}=2.87 \%$, s.d. $=1.16)$ (Figure 1$)$.

\section{Conclusions}

Data on the relationship between hospitalizations and ILI ED visits provide an objective method to describe the burden of disease and to compare severity between influenza seasons, as seen in the leading peak for the 5- to 17-year group in the pandemic spring wave. Further analyses are underway to assess the degree to which (1) the $2009 \mathrm{H} 1 \mathrm{~N} 1$ pandemic impacted these results and (2) comorbid conditions affected susceptibility to and exacerbation of influenza in different age groups.

\section{Acknowledgements}

This paper was presented as an oral presentation at the 2010 International Society for Disease Surveillance Conference, held in Park City, UT, USA, on 1-2 December 2010. 


\title{
Evaluation of the NATO Disease Surveillance System in Kosovo in 2010
}

\author{
J-B Meynard ${ }^{1}$, H Chaudet ${ }^{2}$, L Pellegrin ${ }^{2}$, Y Moreno ${ }^{2}$, O Deboosere ${ }^{2}$, P Mornand ${ }^{2}$, K Pennin ${ }^{3}$, S Stein ${ }^{4}$, B Queyriaux ${ }^{2}$, \\ H-U Holterm ${ }^{5}$, A Zuendorf ${ }^{3}$, J Kocik ${ }^{3}$, R Migliani ${ }^{1}$, X Deparis ${ }^{2}$, and G Texier ${ }^{2}$
}

${ }^{1}$ Ecole du Val-de-Grâce, Paris, France; ${ }^{2}$ Institut de Médecine Tropicale du Service de santé des armées, Marseille, France; ${ }^{3}$ NATO Consultation, Command and Control Agency, The Hague, The Netherlands; ${ }^{4}$ KFOR Headquarters, Pristina, Kosovo; and ${ }^{5}$ Saniaetskommando I, Preventive Medicine Department, Kiel, Germany

E-mail: jb.meynard@wanadoo.fr

\section{Objective}

The new NATO Disease Surveillance System (DSS) was deployed for the second time in Kosovo within the multinational armed forces in 2010 for a 3 days experiment. The objective of the survey was to continue the development of real-time disease surveillance capability for NATO forces, in parallel with the implementation of the NATO Deployment Health Surveillance Centre in Munich in 2010.

\section{Introduction}

The 2010 NATO DSS experiment was the second deployment of the French 'Alerte et Surveillance en Temps Réel' (ASTER) system within a multinational armed task force in real operational conditions. This experiment was scheduled within the ASTER evaluation program (Figure 1), as constructed by French and NATO Armed Forces after several previous works. $^{1-3}$

\section{Methods}

The DSS was set up in May 2010 within the medical facilities of six NATO allied nations taking part in the multinational armed forces task force in Kosovo (KFOR): Austria, Czech Republic, France, Germany, Poland, and United States of America. Each nation received one laptop loaded with software that allowed medical data to be recorded. The recorded data was sent to a national data collection server at KFOR headquarters in Pristina. A permanent communication link was used to send the data simultaneously to the analysis centre level in Munich, where a multinational team was deployed. Real medical data were used, but also simulated data within the Polish medical facility, in order to assess the ability of the analysis level to detect and to manage a natural Norovirus outbreak. Three other types of surveys were realized: a knowledge-attitude-practice survey within the stakeholders, a study of social networks, and a Human Factor study (using Lewis, NASA, and SART questionnaires).

\section{Results}

The Norovirus outbreak was detected in real-time and adapted measures proposed by the analysis level (individual and collective hygiene measures, medical treatment, and adaptation of activities). In all, 42 people took part in the three studies (28 in Kosovo and 14 in Munich). The analysis of the results is currently in progress and will be presented during the congress.

\section{Conclusions}

This evaluation is an intermediary one, it should be strengthened by a final evaluation, promoting continuous improvement, before generalization for all the military NATO deployed units.

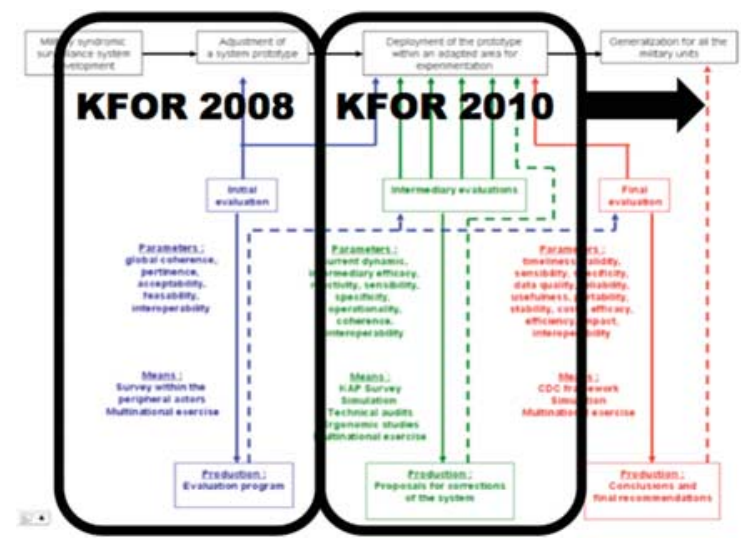

Figure 1 Intermediary evaluation for NATO 'Disease Surveillance System' deployed in Kosovo in November 2010 as a part of the whole NATO evaluation process.

open 2 access This is an Open Access article distributed under the terms of the Creative Commons Attribution licence (http://creativecommons.org/licenses/by/2.5) OPEN - ACCESS which permits unrestricted use, distribution, and reproduction in any medium, provided the original work is properly cited. 


\section{Acknowledgements}

This paper was presented as a poster at the 2010 International Society for Disease Surveillance Conference, held in Park City, UT, USA, on 1-2 December 2010.

\section{References}

1 Meynard J-B, Chaudet H, Green Ad, Jefferson H, Texier G, Webber D, et al. Proposal of a framework for evaluating military

surveillance systems for early detection of outbreaks on duty areas. BMC Public Health 2008;8:146.

2 Jefferson H, Dupuy B, Chaudet H, Texier G, Green Ad, De Lesquen $\mathrm{M}$, et al. Evaluation of a syndromic surveillance for the early detection of outbreaks among military personnel in a tropical country. J Public Health 2008;30:375-83.

3 Meynard J-B, Chaudet H, Varcol C, Decker E, Andresen S, Holterm $\mathrm{H}-\mathrm{U}$, et al. Evaluation of the NATO Disease Surveillance System by its users in Kosovo in 2008. Mil Med 2010;175:466-8. 


\title{
Creating a fast and flexible syndromic surveillance reporting system
}

\author{
J Morin ${ }^{1,2}$, A Ali $^{3}$, D Ferguson ${ }^{2}$, RG Gutierrez ${ }^{4}$, A Riley ${ }^{4}$, N Vinson ${ }^{2}$, S McClinton ${ }^{1}$, C McDermaid ${ }^{3}$, K Ali ${ }^{5}$, J Martin ${ }^{2}$, and \\ RF Davies ${ }^{1}$
}

\author{
${ }^{1}$ University of Ottawa Heart Institute, Ottawa, Ontario, Canada; ${ }^{2}$ National Research Council of Canada Institute for Information Technology, \\ Ottawa, Ontario, Canada; ${ }^{3}$ Ottawa Public Health (OPH), Ottawa, Ontario, Canada; ${ }^{4}$ StataCorp LP, College Station, TX, USA; and ${ }^{5}$ AMITA \\ Corporation, Ottawa, Ontario, Canada \\ E-mail: jason.morin@nrc.gc.ca
}

\section{Objective}

The objective of this study was to create and evaluate a system that uses customized scripts developed for commercial off-the-shelf (COTS) statistical and GIS software to (1) analyze syndromic data and produce regular reports to public health epidemiologists, containing the information they would need to detect and manage an ILI outbreak, and (2) facilitate the generation more detailed analyses relevant to specific situations using these data.

\section{Introduction}

Syndromic surveillance systems significantly enhance the ability of Public Health Units to identify, quantify, and respond to disease outbreaks. Existing systems provide excellent classification, identification, and alerting functions, but are limited in the range of statistical and mapping analyses that can be done. Currently available COTS statistical and GIS packages provide a much broader range of analytical and visualization tools, as well as the capacity for automation through user-friendly scripting languages. This study retrospectively evaluates the use of these packages for surveillance using syndromic data collected in Ottawa during the $2009 \mathrm{pH} 1 \mathrm{NI}$ outbreak.

\section{Methods}

Four Ottawa area hospitals have been reporting chief complaint and demographic data to Ottawa Public Health using a RODS-based syndromic surveillance system (ASSET) since January 2009. (ASSET is based on the University of Pittsburgh's Real-time Outbreak and Disease Surveillance system (http://www.rods.pitt.edu)). During the 2009 pH1N1 pandemic, a team comprised of epidemiologists, health care researchers, and NRC HCI specialists recognized the need for an automated syndromic reporting system that would free epidemiologists for other tasks. The team designed specific reports by developing customized scripts using Stata and ArcGIS. Data from ASSET were parsed using an ILI classifier (NRC) and processed using these scripts.

\section{Results}

The resulting ILI Watch report automatically produces epicurves, exploratory data analyses, aberration detection graphs, and color-coded maps that replace daily reports, which previously had to be generated manually. The system also makes the syndromic data set easily available to epidemiologists, and facilitates follow-up analyses. The system is currently in production in the Ottawa area (Figure 1).

\section{Conclusions}

An automated system developed using state-of-the-art COTS software can reduce the time and resource burdens on epidemiologists and IT staff during an outbreak by

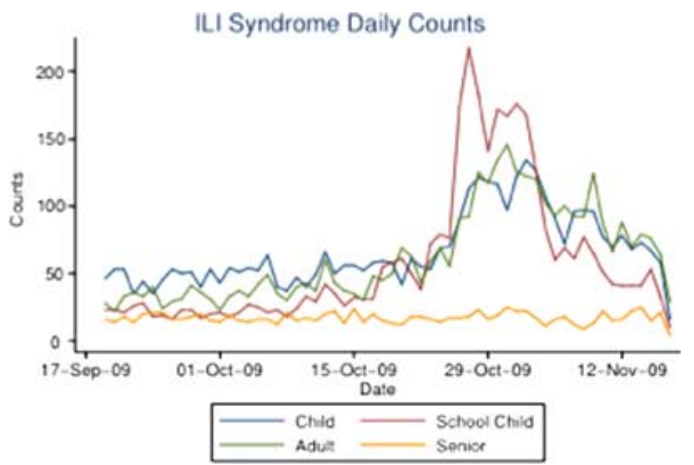

Figure 1 Sample data from the ASSET ILI Watch. Data is categorized by age group, and demonstrates both the high prevalence of ILI in children in general and school-aged children in particular, and the nominal infection rates seen in people aged $\geqslant 60$ years.

open -2 access This is an Open Access article distributed under the terms of the Creative Commons Attribution licence (http://creativecommons.org/licenses/by/2.5) OPEN - ACCESS which permits unrestricted use, distribution, and reproduction in any medium, provided the original work is properly cited. 
automatically producing customized reports. It also provides the flexibility needed to rapidly meet changing information needs by providing a user-friendly scripting template for ad hoc analyses.

\section{Acknowledgements}

This paper was presented as a poster at the 2010 International Society for Disease Surveillance Conference, held in Park City, UT, USA, on 1-2 December 2010. 


\title{
Comparison of respiratory, febrile and influenza-like illness syndromes to detect laboratory-reported H1N1 and RSV, Influenza Season 2009-10, New York City
}

\author{
E Westheimer, TQ Nguyen, M Paladini, D Weiss, and S Balter
}

New York City Department of Health and Mental Hygiene, New York, NY, USA

E-mail: tnguyen@health.nyc.gov

\section{Objective}

To determine the correlation of the influenza-like illness (ILI) syndrome with laboratory-confirmed $\mathrm{H} 1 \mathrm{~N} 1$ and respiratory syncytial virus (RSV) during the October 2009 to March 2010 H1N1 season in New York City (NYC).

\section{Introduction}

The NYC Department of Health and Mental Hygiene (DOHMH) monitors visits daily from 49 of 54 NYC emergency departments (EDs), capturing 95\% of all ED visits. ED visits for ILI have reflected influenza activity in NYC, ${ }^{1}$ better than the more broadly defined fever/flu (FF) and respiratory (Resp) syndromes, but the correlation with H1N1 is unknown.

Laboratory-confirmed influenza and RSV were made reportable in NYC in February 2008. DOHMH receives electronic reports of positive tests.
As part of 2009-10 influenza surveillance, five hospitals were selected for 'sentinel' surveillance of hospitalized influenza cases, to test all patients with a respiratory condition for influenza. Sentinel hospitals ensured that patient medical record numbers (MRNs) were in the daily ED syndromic file and in the electronic laboratory reports.

\section{Methods}

Sentinel hospital ED visits were matched by MRN to corresponding cases of influenza and RSV reported electronically for 1 October 2009 through 31 March 2010. Trends were assessed citywide, across sentinel hospitals, and by hospital and age groups. Correlation coefficients were calculated.

\section{Results}

Between 1 October 2009 and 31 March 2010, the five sentinel hospitals represented $13.6 \%$ of 1,939,417 citywide ED visits. Of

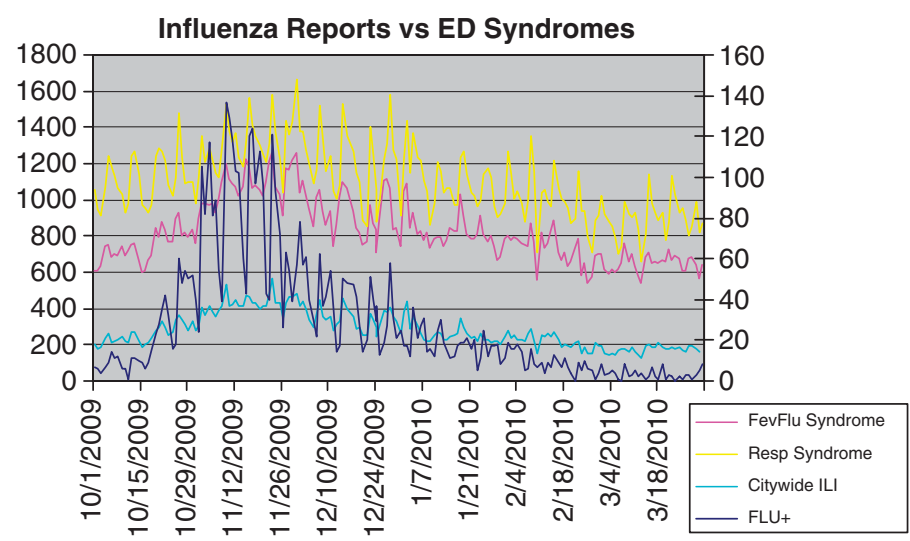

\section{CONCLUSIONS}

Figure 1 Weekly citywide ILI ED visits vs citywide laboratory reports of influenza and RSV, October 2009-March 2010, New York City. This is an Open Access article distributed under the terms of the Creative Commons Attribution licence (http://creativecommons.org/licenses/by/2.5)
open 2 access permits unrestricted use, distribution, and reproduction in any medium, provided the original work is properly cited. 
the sentinel ED visits, 24,100 (9\%) were categorized as FF, 29,859 (11\%) were Resp, and 4996 (2\%) were ILI; 21\% of FF visits and $10 \%$ of Resp visits also met the ILI definition.

During the same period, the sentinel hospitals reported $550(11 \%)$ of 5137 unique laboratory reports of influenza and 1348 (27\%) of 5030 RSV reports. Citywide, ILI correlated best with influenza reports $(0.82)$, followed by FF $(0.78)$ and Resp (0.70) (see Figure 1).

With the sentinel data, 499 influenza reports and 1114 RSV reports were matched by MRN to an ED visit. The majority of influenza reports matched to the FF (54\%) and Resp (23\%) syndromes; $14 \%$ were ILI. RSV cases matched similarly to FF (41\%), Resp (40\%), and ILI (10\%).

\section{Conclusions}

Although influenza cases were classified primarily as FF or Resp, the correlation of ILI with influenza was higher than the other syndromes. Therefore, ILI still best reflects influenza trends but not the magnitude of illness.
ILI trends enable citywide monitoring of influenza, but variations by age group and hospital catchment suggest that fine-tuning syndromes to better represent influenza and RSV activity is possible. Data matching was limited to only five hospitals, but demonstrates that matching ED visits to laboratory reports could enhance syndrome definitions and modeling.

\section{Acknowledgements}

This paper was presented as a poster at the 2010 International Society for Disease Surveillance Conference, held in Park City, UT, USA, on 1-2 December 2010.

\section{Reference}

1 Olson DR, Heffernan RT, Paladini M, Konty K, Weiss D, Mostashari F. Monitoring the impact of influenza by age: emergency department fever and respiratory complaint surveillance in New York City. PLoS Medicine 2007;4:1349-61. 


\title{
Early Aberration Reporting System (EARS) update: present and future
}

\author{
G Rainisch, and K Hayashi \\ Centers for Disease Control and Prevention (CDC), Atlanta, GA, USA \\ E-mail: buu8@cdc.gov
}

\section{Objective}

The objective of this poster is to highlight recent upgrades to the Early Aberration Reporting System (EARS) software (US Centers for Disease Control and Prevention, EARS Program, MS C-18, Atlanta, GA, USA), and identify features planned for future releases.

\section{Introduction}

Early Aberration Reporting System (EARS) is a freeware surveillance tool that can be downloaded from the Center for Disease Control and Prevention's website (http:// emergency.cdc.gov/surveillance/ears/). It was designed for quick set-up and customization for automated monitoring of emergency department and other syndromic data sources, including, but not limited to, 911 calls, school absenteeism, and over-the-counter medication sales. The United States' city, county, state health departments, and various international public health organizations, use EARS software to conduct daily, near-real time surveillance of conditions easily defined by patient-reported complaints, and physician diagnoses (for example, influenza-like illness, gastroenteritis, asthma, heat-related illness). It is also used to conduct suspect case finding during outbreaks, natural disaster responses, verify that potential threats are not manifested in communities, and for supporting ad hoc analyses and research.

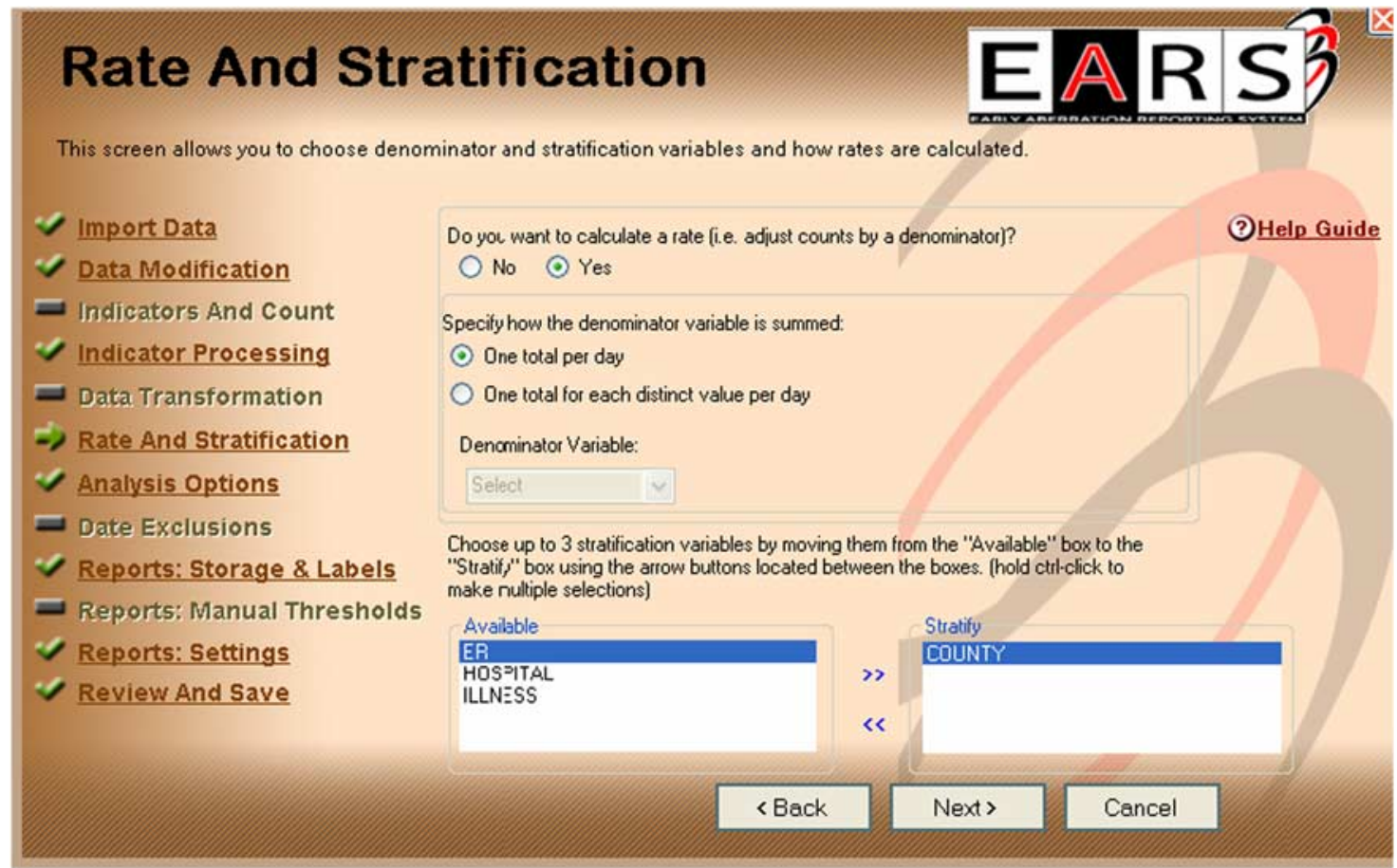

Figure 1 Screen example of the EARS-SAS version5.0 graphical user interface.

This is an Open Access article distributed under the terms of the Creative Commons Attribution licence (http://creativecommons.org/licenses/by/2.5) OPEN - ACCESS which permits unrestricted use, distribution, and reproduction in any medium, provided the original work is properly cited. 


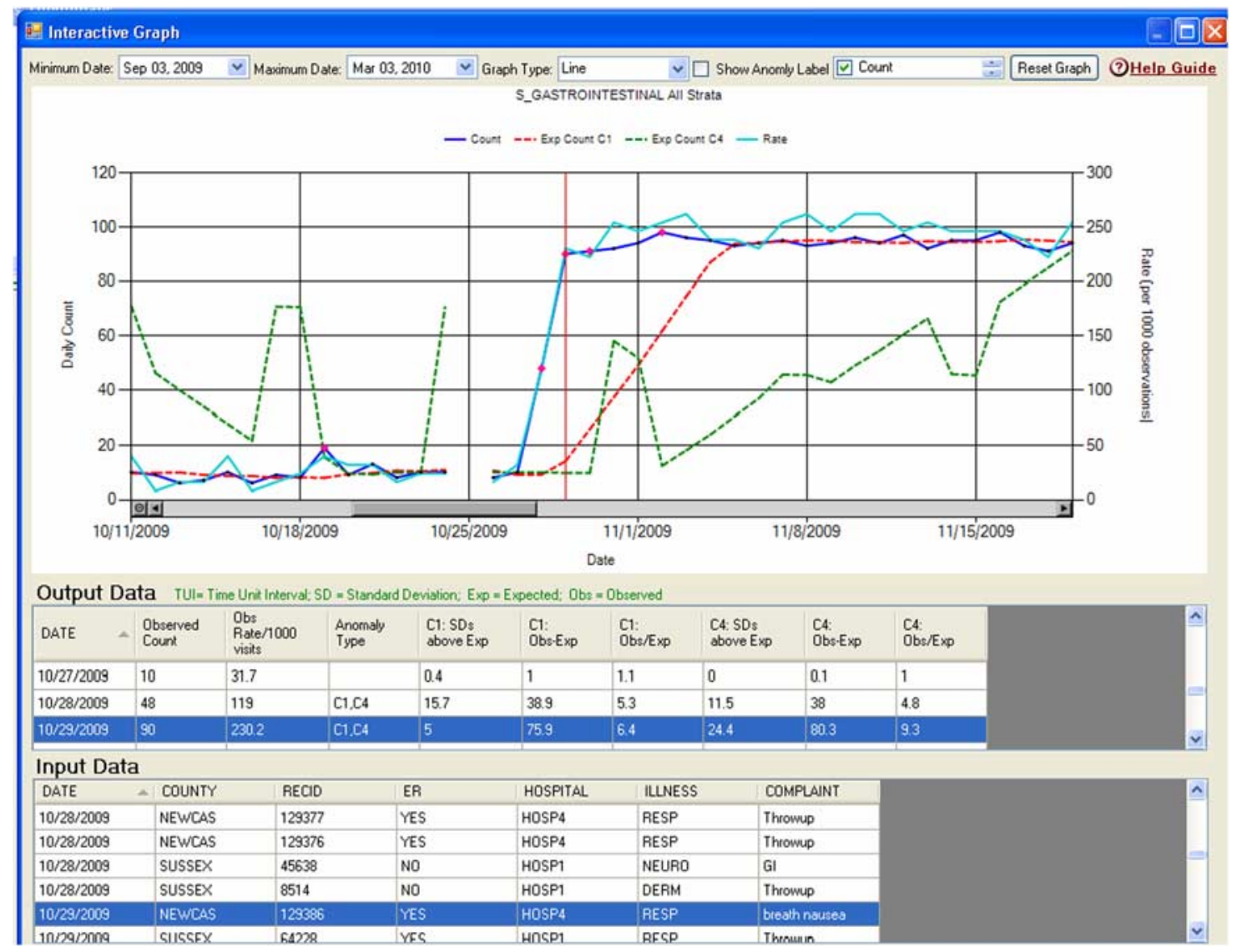

Figure 2 Screen example of the EARS-SAS version5.0 interactive graphs output.

\section{Methods}

EARS is undergoing significant changes focused on reducing processing times and expanding user-defined customization options. Installation and set-up have been simplified through the use of a 'wizard-like' graphical user interface that significantly restricts user-generated errors (Figure 1). Anomaly detection algorithms are being modified to increase both sensitivity and specificity (without increasing the alert rate) through use of a minimum standard deviation, a longer baseline/comparison period, an adjustment for total visits (or other denominator), and an option for stratifying expected value calculations by days of the week. ${ }^{1}$ Output filtering options, based on both empirical and epidemiological criteria, are being added to allow the user to further manage the alert rate (Figure 2).

\section{Results}

Future enhancements include writing EARS in other languages to support an increasing international user-base. Rewrites will be on the basis of open-source tools that will not require Microsoft Excel (Microsoft Corporation, Redmond, WA, USA) or SAS (Institute Inc., Cary, NC, USA) to run. Optional plug-ins will enable users to format and automate submission of aggregate-level data summaries to other systems (for example, DiSTRIBuTE, http://www. isdsdistribute.org/). The EARS program plans to also explore increasing EARS compatibility with varying data types, such as diagnoses and laboratory data through data-specific modules (based on the design of the current EARS Indicator Processing feature).

\section{Conclusions}

The potential utility and access to electronic health records, and syndromic data sources are expected to continue to expand. The EARS tool continues to improve to keep up with this demand. Collaboration on EARS design, with state and local public health departments, is a crucial component to its continued successful development.

\section{Acknowledgements}

This paper was presented as a poster at the 2010 International Society for Disease Surveillance Conference, held in Park City, UT, USA, on 1-2 December 2010.

\section{Reference}

1 Tokars JI, Burkom H, Xing J, English R, Bloom S, Cox K, et al. Enhancing time-series detection algorithms for automated biosurveillance. Emerg Infect Dis 2009;15:4. 


\title{
Description of the quality of public health case reports received at a local health department and potential impact on workflow
}

\author{
D Rajeev ${ }^{1}$, A Price ${ }^{2}, \mathrm{~K} \mathrm{Schnirel}^{2}$, M Hill ${ }^{2}$, C Staes ${ }^{1}$, and I Risk ${ }^{2}$ \\ ${ }^{1}$ Department of Biomedical Informatics, University of Utah, Salt Lake City, UT, USA; and ${ }^{2}$ Salt Lake Valley Health Department, Salt Lake City, \\ UT, USA \\ E-mail: deepthi.rajeev@hsc.utah.edu
}

\section{Objective}

This paper describes a comparison study conducted to identify quality of reportable disease case reports received at Salt Lake Valley health department (SLVHD) in 2009 and 2010.

\section{Introduction}

When a reportable condition is identified, clinicians and laboratories are required to report the case to public health authorities. These case reports help public health officials to make informed decisions and implement appropriate control measures to prevent the spread of disease. Incomplete or delayed case reports can result in new occurrences of disease that could have been prevented. To improve the disease reporting and surveillance processes, the Utah Department of Health is collaborating with Intermountain Healthcare and the University of Utah to electronically transmit case reports from healthcare facilities to public health entities using Health Level Seven v2.5, SNOMED CT, and LOINC. ${ }^{1}$ As part of the Utah Center of Excellence in Public Health Informatics, we conducted an observation study in 2009 to identify metrics to evaluate the impact of electronic systems. ${ }^{2}$ We collected baseline data in 2009 and in this paper we describe preliminary results from a follow-up study conducted in 2010.

\section{Methods}

We conducted two observation studies of the workflow associated with processing case reports at SLVHD, including from 6 July 2009 to 13 July 2009 and 7 July 2010 to 21 July 2010. The 2009 study occurred during the H1N1 outbreak. The studies involved direct observations of the workflow of the triage nurse at SLVHD. To ensure we were capturing the quality of the reports received initially at the health department, we used a data collection form to document whether certain core data elements such as 'patient address', 'patient telephone number', 'hospitaliza- tion status', 'physician notes' and so on, were missing. Currently, we are extracting data from the Utah statewide surveillance system (UT-NEDSS) to compute the 'time to diagnosis of a case', 'reporting time delay', 'time to triage a report', 'time until the start of case investigation' and so on,

\section{Results}

In 2009 ( $n=380$ reports) and 2010 ( $n=322$ reports), there were similar proportion of out-of-county reports (23 and 29\%, respectively) and duplicate reports (19 and 20\%, respectively). The quality of data in reports received in 2009 and in 2010 is described in Table 1. In 2009, Chlamydia, Influenza related cases, and Salmonella contributed to $68 \%$ of the reports and in 2010, Chlamydia, Giardia, and Salmonella represented 60\% of the reports. The analysis of the timeliness of the reporting process is currently underway.

\section{Discussion}

The processing of out-of-county and duplicate reports continues to be a burden on the triage nurse. The hospitalization status and physician notes were more complete in 2009 than in 2010. Patient telephone number and patient address are generally required for all diseases but

Table 1 Comparison of quality of key data elements in reports received at SLVHD in 2009 and 2010

\begin{tabular}{|c|c|c|}
\hline \multirow[t]{2}{*}{ Data element } & \multicolumn{2}{|c|}{$\begin{array}{c}\% \text { of (updated } / \text { new) reports with information } \\
\text { included }\end{array}$} \\
\hline & $2009(\%)$ & $2010(\%)$ \\
\hline Patient telephone number & 82 & 81 \\
\hline Patient address $^{\mathrm{a}}$ & 79 & 61 \\
\hline Hospitalization status $^{a}$ & 86 & 20 \\
\hline Physician Notes $^{a}$ & 82 & 8 \\
\hline
\end{tabular}

${ }^{\text {a }}$ Significant difference at $\alpha=0.05$.

This is an Open Access article distributed under the terms of the Creative Commons Attribution licence (http://creativecommons.org/licenses/by/2.5) OPEN - ACCESS which permits unrestricted use, distribution, and reproduction in any medium, provided the original work is properly cited. 
hospitalization status and physician notes are not typically provided and/or required with the disease report. However, during the H1N1 outbreak these data were requested with the report to quickly identify routes of exposure to reduce spread. The results demonstrate the challenges and burden for public health to obtain additional data elements such as hospitalization status and physician notes.

\section{Acknowledgements}

This paper was presented as a poster at the 2010 International Society for Disease Surveillance Conference, held in Park City, UT, USA, on 1-2 December 2010. Funding: (Grant\#
1PO1HK000069-01) Rocky Mountain Center for Translational Research in Public Health Informatics, NLM Training grant \# 5T15LM007124 (DR).

\section{References}

1 Rajeev D, Staes CJ, Evans RS, Mottice S, Rolfs R, Samore MH, et al. Development of an electronic public health case report using HL7 v2.5 to meet public health needs. J Am Med Inform Assoc (JAMIA) 2010;17:34-41.

2 Rajeev D, Zeller R, Price A, Reid J, Staes CJ, Risk I. Evaluating the Impact of Electronic Disease Surveillance Systems on Local Health Department Work Processes. Public Health Information Network (PHIN); Atlanta, GA, 2009. 


\title{
Asthma patterns in Boston emergency department visits for children age five and under
}

\author{
M Rennick ${ }^{1}$, J Gunn ${ }^{1}$, M Donovan ${ }^{1}$, and J Salvia ${ }^{2}$ \\ ${ }^{1}$ Boston EMS, Boston Public Health Commission, Boston, MA, USA; and ${ }^{2}$ Communicable Disease Control Division, Raleigh, NC, USA \\ E-mail: mrennick@bphc.org
}

\section{Objective}

The objective of this study is to report on the use of syndromic surveillance data to describe seasonal patterns of asthma and health inequities among Boston residents, age five and under.

\section{Introduction}

The burden of asthma on the youngest children in Boston is largely characterized through hospitalizations and self-report surveys. $^{1}$ Hospitalization rates are highest in Black and Hispanic populations under age five. A study of children living in Boston public housing showed significant risk factors, including obesity and pest infestation, with less than half of the study population being prescribed daily medication. ${ }^{2}$

Information on asthma visits for children $\leqslant 5$ years old was requested by the Boston Public Health Commission Community Initiatives Bureau. The information is being used to establish a baseline for an integrated Healthy Homes Program that includes pest management and lead abatement. There is limited experience in using syndromic surveillance data for chronic disease program planning.

\section{Methods}

The Boston syndromic surveillance system receives demographics, chief complaints, and disposition. To assess asthma visits in the emergency departments (ED) from 1 January 2008-19 June 2010, an asthma syndrome was built to catalog the following key words (and appropriate variations) from the chief complaint field: asthma, reactive airway disease, and wheezing. This was then restricted to all Boston residents, ages five and under, and compared with all ED visits of the same ages and residency. Syndrome counts were further stratified by race/ethnicity, geographic neighborhoods, gender, and disposition.

Boston Emergency Medical Service transports coded as likely asthma in the electronic trip sheet for Boston residents, ages five and under were also obtained.

\section{Results}

Asthma ED visits occurred in a seasonal pattern in Boston with highest levels of activity in September to early October in both 2008 and 2009 ( $\max =10.6 \%$ of all visits for week ending 20 September 2008), and was correlated with an increase in Emergency Medical Service transports $\left(\mathrm{R}^{2}=0.23\right)$. (Figure 1).

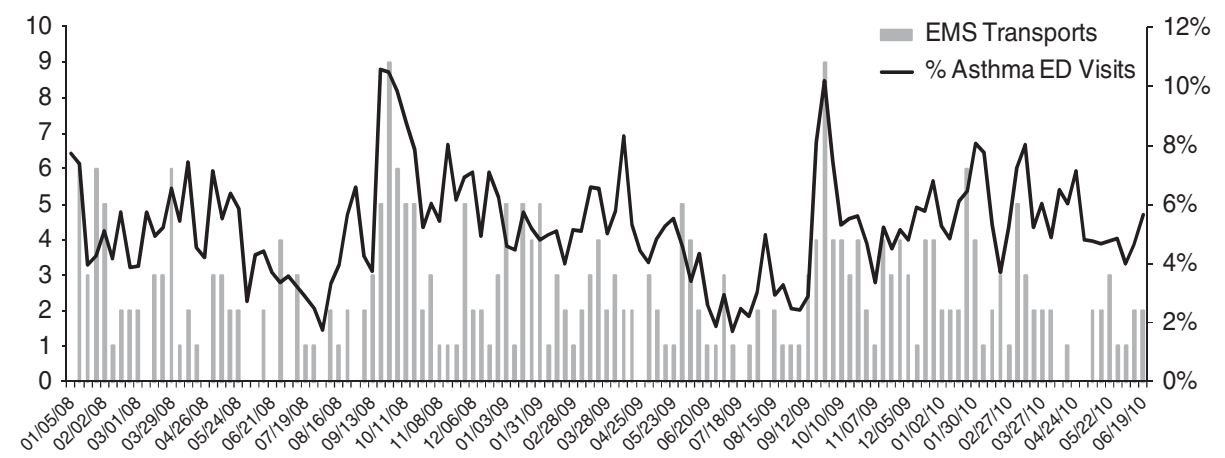

Figure 1 Weekly percent asthma of all Boston ED visits $\leqslant 5$ years old (line) overlayed with weekly count of EMS transports for asthma patients $\leqslant 5$ years old (bars), 1 January 2008-19 June 2010. This is an Open Access article distributed under the terms of the Creative Commons Attribution licence (http://creativecommons.org/licenses/by/2.5)
open which permits unrestricted use, distribution, and reproduction in any medium, provided the original work is properly cited. 


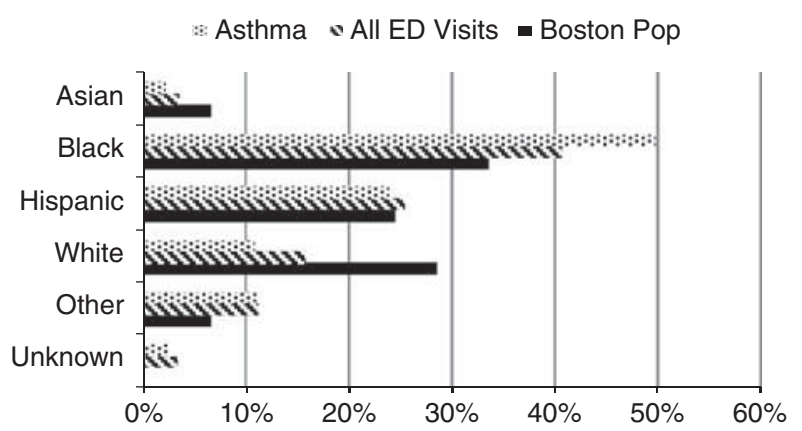

Figure 2 Race/ethnicity distribution of ED visits for asthma, and for all visits compared with Boston population $\leqslant 5$ years old, 1 January 2008-19 June 2010. Fifty percent of asthma patients are Black, $41 \%$ of all ED patients are Black, 34\% of the age-specific Boston population are Black (2000 Census).

From 1 January 2008-19 June 2010, Black children were nearly twice as likely to present to an ED for an asthma syndrome visit compared with White children (odds ratio $(\mathrm{OR})=1.84(1.65,2.06))$; Hispanic children were $40 \%$ more likely $(\mathrm{OR}=1.40(1.24,1.57))$ than White children to present at an ED for an asthma visit. Males accounted for $64 \%$ of all asthma syndrome visits compared with $54 \%$ for all ED visits. Between 29 November 2009 and 19 June 2010, 25\% of the ED asthma syndrome visits required hospitalization. (Figure 2).

\section{Conclusions}

The Boston Public Health Commission syndromic surveillance system provided information on ED asthma syndrome visits for children $\leqslant 5$ years old. Seasonal patterns in asthma syndrome visits correlated with Emergency Medical Service data with peak activity in September. Environmental triggers including returning to school (either patients or siblings) may be associated with increased activity in September. A flexible chief complaint coding system with the availability of demographic information that included race/ethnicity was essential to providing timely baseline information for program planning.

\section{Acknowledgements}

This paper was presented as a poster at the 2010 International Society for Disease Surveillance Conference, held in Park City, UT, USA, on 1-2 December 2010.

\section{References}

1 Boston Public Health Commission Research and Evaluation Office. Health of Boston 2009. Boston Public Health Commission Research and Evaluation Office. Boston, MA, 2009.

2 Levy J, Welker-Hood LK, Clougherty JE, Dodson RE, Steinbach S, Hynes HP. Lung function, asthma symptoms, and quality of life in public housing in Boston: a case-series analysis. Environ Health 2004;3:13. 


\section{Evaluating the use of syndromic surveillance for the detection of influenza-like illness in Salt Lake County, Utah}

J Robertson, and M Hill

Salt Lake Valley Health Department, Salt Lake City, UT, USA

E-mail: jrobertson@slco.org

\section{Objective}

The objective of this study is to compare the performance of syndromic surveillance with the United States Outpatient Influenza-like Illness Surveillance Network (ILINet), for the detection of influenza-like illness (ILI) during the fall 2009 wave of H1N1 influenza in Salt Lake County.

\section{Introduction}

Salt Lake Valley Health Department uses syndromic surveillance to monitor ILI activity as part of a comprehensive influenza surveillance program that includes pathogenspecific surveillance, sentinel surveillance, school absenteeism and pneumonia, and influenza mortality. During the 2009 spring and fall waves of novel H1N1 influenza, sentinel surveillance became increasingly burdensome for both community clinics and Salt Lake Valley Health Department, and an accurate, more efficient method for ILI surveillance was needed. One study found that syndromic surveillance performed, as well as a sentinel provider system in detecting an influenza outbreak ${ }^{1}$ and syndromic surveillance is currently used to monitor regional ILI in the United States. ${ }^{2}$

\section{Methods}

Data were collected from 30 August 2009 to 26 December 2009. Weekly reported hospitalized H1N1 cases were summed from daily lab and provider reports. Daily sentinel provider ILI, defined as fever $\geqslant 100.4^{\circ} \mathrm{F}$, cough and/or sore throat, and total patient visits were summed from 12 sentinel sites, and used to calculate weekly percentages of sentinel provider ILI. Daily text-based chief complaint data from 15 syndromic sites (emergency departments and urgent care centers) were obtained from EpiCenter ${ }^{3}$ (funds provided by Utah Department of Health), mapped to an ILI disease category, defined as 'fever' and 'cough' or 'sore throat,' and summarized using the Early Aberration Reporting System (EARS). ${ }^{4}$ Daily ILI and total patient visits were used to calculate weekly percentages of EARS/syndromic ILI. Spearman correlation analysis was performed to determine the relationships between EARS/syndromic ILI, sentinel provider ILI, and reported hospitalized H1N1 case counts for all ages and each ILI age group.

\section{Results}

Overall, weekly percentage of EARS/syndromic ILI strongly correlated with both weekly percentage of sentinel provider ILI $(r=0.93, P<0.0001)$, and weekly reported hospitalized H1N1 case counts $(r=0.97, P<0.0001)$ (Figure 1$)$. Strong correlations of EARS/syndromic ILI, with sentinel provider ILI and H1N1 case counts were also evident in the 0-4, 5-24, 25-49, and 50-64 age groups.

\section{Conclusions}

These results suggest that syndromic surveillance is an accurate method for ILI surveillance. Salt Lake Valley Health

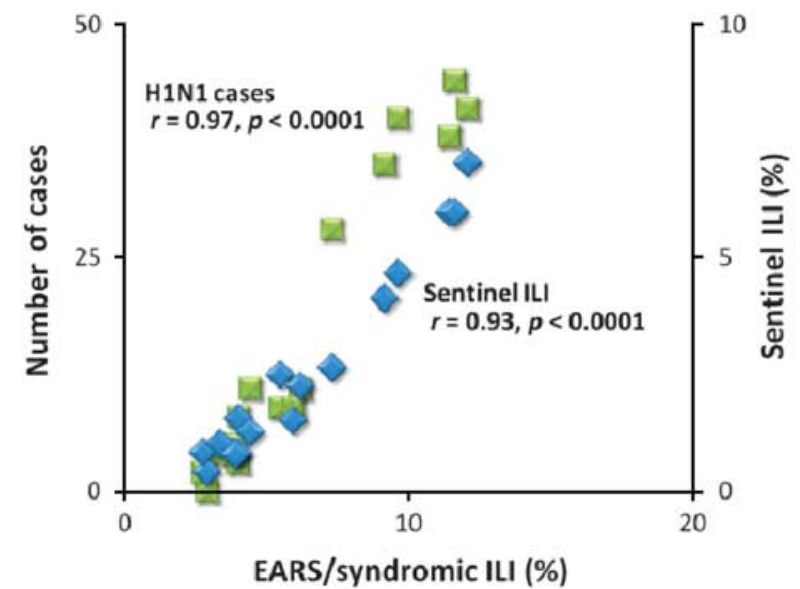

Figure 1 Correlation of EARS/syndromic \% ILI with reported hospitalized H1N1 influenza cases (green), and sentinel \% ILI (blue).

This is an Open Access article distributed under the terms of the Creative Commons Attribution licence (http://creativecommons.org/licenses/by/2.5) OPEN - ACCESS which permits unrestricted use, distribution, and reproduction in any medium, provided the original work is properly cited. 
Department plans to collect ILI data from both syndromic and sentinel surveillance systems in future influenza seasons and establish an epidemic threshold percentage for syndromic ILI, with the eventual goal of replacing sentinel ILI surveillance with syndromic ILI surveillance.

\section{Acknowledgements}

This paper was presented as a poster at the 2010 International Society for Disease Surveillance Conference, held in Park City, UT, USA, on 1-2 December 2010.

\section{References}

1 Ritzwoller DP, Kleinman K, Palen T, Abrams A, Kaferly J, Yih W, et al. Comparison of syndromic surveillance and a sentinel provider system in detecting an influenza outbreak-Denver, Colorado, 2003. MMWR Morb Mortal Wkly Rep 2005;54 (Suppl): 151-6.

2 International Society for Disease Surveillance (ISDS), DiSTRIBuTE project. http://isdsdistribute.org/index.php.

3 Health Monitoring Systems, Inc, EpiCenter, v 2.6.3. http://www. hmsinc.com/service/epicenter.html.

4 Centers for Disease Control and Prevention, Division of Preparedness and Emerging Infections, Early Aberration Reporting System v 45. http://www.bt.cdc.gov/surveillance/ears/. 


\title{
School absenteeism and emergency department ILI rates in King County, WA 2003-2009
}

\author{
C Rodriguez ${ }^{1,2}$, A Baer ${ }^{1,2}$, J Baseman², I Painter ${ }^{2}$, D Yanez², T Koepsell², and JS Duchin ${ }^{1,2}$ \\ ${ }^{1}$ Public Health - Seattle \& King County (PHSKC), Seattle, WA, USA; and ${ }^{2}$ University of Washington, Seattle, WA, USA \\ E-mail: carla.rodriguez@kingcounty.gov
}

\section{Objective}

To describe the relationship between emergency department (ED) visits for influenza-like-illness (ILI) and absenteeism among school-aged children.

\section{Background}

Absenteeism is regarded as an expedient and responsive marker of illness activity. It has been used as a health outcome measure for a wide spectrum of exposures and as an early indicator of influenza outbreaks. ${ }^{1}$ A handful of studies have described its validity compared with traditional 'goldstandards' for influenza and ILI. ${ }^{2,3}$ We sought to further quantify the relationship between ED ILI and school absenteeism because absenteeism, as it relates to illness, and subsequent loss in productivity and wages for parents, school staff and children, is an important public health outcome.

\section{Methods}

Data were drawn from Public Health-Seattle \& King County's syndromic surveillance system and included the weekly number absent by school and the weekly number of ILI visits in the county. Eighteen of nineteen public school districts and 18 of 19 hospitals contributed data for schoolaged children from 2003-2009. An ecological study design and generalized estimating equations for Poisson models were used to measure the marginal association between county-wide (log) ED ILI rates and school absenteeism over time, using school enrollment as an offset. Models included adjustment by school level, influenza season, (log) average baseline absenteeism count, pre- and post-holiday weeks, time and confounding by diarrheal illness. The 2003-04 and 2008-09 school years were analyzed separately because of distinct patterns in respiratory virology during those years. Sensitivity analyses for the number of ED visits per school, simulated using a normal distribution for a range of means, were conducted to test the reliability of our results. Signals, representing excess events, in both ED and absenteeism data streams were generated using the cumulative sum (CUSUM) method and compared with virology data from the University of Washington Clinical Virology Laboratory. Virology data were also used to define the start and peak of viral epidemics, including influenza.

\section{Results}

During seasonal influenza periods from 2003-08, a 3-fold increase in county-wide ED ILI rate among children aged 5-9 years was associated with 18\% (95 CI: 16,20\%) higher absenteeism in elementary schools. Associations were similar among elementary and middle school-aged children, and greater than those observed in high schools. During the spring 2009 H1N1 pandemic, a 3-fold increase in ED ILI rate was associated with 38\% (95 CI: 33,43\%) higher absenteeism in elementary schools. We generally observed only weak associations between absenteeism and ILI during noninfluenza periods from 2003-08 and outside the pandemic period of 2009. Predicted absenteeism rates were less variable than ILI rates, but still sensitive to the rise and peak of ED ILI and influenza epidemics. ED ILI signals were better correlated with virology data for the start and peak of influenza season than predicted absenteeism.

\section{Conclusions}

To our knowledge, this is the first study to quantify the degree to which absenteeism increases when ED ILI activity increases over several viral epidemics. We also observed improved temporal correlation of ED ILI signals with influenza data over predicted absenteeism, supporting the use of ED ILI as a predictor for school absenteeism. Because data on ED visits were only available at the county-level, we were unable to control for potential confounding by ED visits at the school level. However, our results were robust to sensitivity analyses. This is a first step towards understanding the validity of school absenteeism to describe ILI activity. Future studies that better control for the effect of schoollevel ED visits on absenteeism and test the predictive ability

This is an Open Access article distributed under the terms of the Creative Commons Attribution licence (http://creativecommons.org/licenses/by/2.5) OPEN - ACCESS which permits unrestricted use, distribution, and reproduction in any medium, provided the original work is properly cited. 
of absenteeism for ILI are needed to verify and expand upon our results.

\section{Acknowledgements}

This paper was presented as an oral presentation at the 2010 International Society for Disease Surveillance Conference, held in Park City, UT, USA, on 1-2 December 2010.

\section{References}

1 Weitzman M. School absence rates as outcome measures in studies of children with chronic illness. J Chronic Dis 1986;39: 799-808.

2 Glezen WP. Emerging infections: pandemic influenza. Epidemiol Rev 1996;18:64-76.

3 Quenel P, Dab W, Hannoun C, Cohen JM. Sensitivity, specificity and predictive values of health service based indicators for the surveillance of influenza A epidemics. Int J Epidemiol 1994;23: 849-55. 


\title{
Impact of alternate diagnoses on the accuracy of influenza-like illness case definition used for H1N1 screening in the emergency department
}

\author{
D Rumoro ${ }^{1}$, J Silva ${ }^{1}$, S Shah ${ }^{1}$, J Bayram ${ }^{1}$, M Hallock ${ }^{1}$, G Gibbs ${ }^{1}$, M Waddell ${ }^{2}$, and K Thomas ${ }^{1}$ \\ ${ }^{1}$ Rush University Medical Center, Chicago, IL, USA; and ${ }^{2}$ Pangaea Information Technologies, Chicago, IL, USA \\ E-mail: dino_rumoro@rush.edu
}

\section{Objective}

This study investigates the impact of alternate diagnoses on the accuracy of the Centers for Disease Control and Prevention's (CDC) case definition for influenza-like illness (ILI) when used as a screening tool for influenza A (H1N1) virus during the 2009 pandemic, and the implications for public health surveillance.

\section{Introduction}

In June 2009, the CDC defined a confirmed case of H1N1 as a person with an ILI and laboratory confirmed novel influenza A H1N1 virus infection. ${ }^{1}$ ILI is defined by the CDC as fever and cough and/or sore throat, in the absence of a known cause other than influenza. ${ }^{1}$ ILI cases are usually reported without accounting for alternate diagnoses (that is, pneumonia). Therefore, evaluation is needed to determine the impact of alternate diagnoses on the accuracy of the ILI case definition.

\section{Methods}

This is a retrospective cross-sectional study design conducted from September 5, 2009 to May 5, 2010, at an emergency department of a large urban tertiary care academic medical center. During this period, 32,922 patients were seen, of which 1233 were tested for H1N1 using Polymerase Chain Reaction (PCR) testing for respiratory viruses. Geographic Utilization of Artificial Intelligence in Real-Time for Disease Identification and Notification (GUARDIAN), a syndrome surveillance program, ${ }^{2}$ was utilized to assign ILI status to each patient.

Positive predictive value (PPV), negative predictive value (NPV), sensitivity, and specificity were calculated with and without consideration of the following alternate diagnoses: pneumonia, respiratory syncytial virus, infectious mononucleosis, and streptococcal pharyngitis. Positive laboratory results, in addition to ICD-9 codes, were used to detect these alternate diagnoses. McNemar's and $\chi^{2}$-test were then used to compare the results for statistical significance (Table 1).
Table 1 Positive and negative predictive value and sensitivity and specificity of the ILI case definition to detect H1N1 cases

\begin{tabular}{|c|c|c|c|c|c|c|c|}
\hline \multirow[t]{2}{*}{ ILI status } & \multicolumn{3}{|c|}{ H1N1 status } & \multirow{2}{*}{$\begin{array}{l}\text { PPV } \\
\text { (\%) }\end{array}$} & \multirow{2}{*}{$\begin{array}{l}N P V \\
(\%)\end{array}$} & \multirow{2}{*}{$\begin{array}{c}\text { Sensitivity } \\
(\%)\end{array}$} & \multirow{2}{*}{$\begin{array}{l}\text { Specificity } \\
\text { (\%) }\end{array}$} \\
\hline & & No & Yes & & & & \\
\hline \multirow{2}{*}{$\begin{array}{l}\text { Including } \\
\text { alternate } \\
\text { diagnoses }\end{array}$} & No & 439 & 21 & 17.2 & 95.4 & 86.4 & 40.7 \\
\hline & Yes & 640 & 133 & & & & \\
\hline \multirow{2}{*}{$\begin{array}{l}\text { Excluding } \\
\text { alternate } \\
\text { diagnoses }\end{array}$} & No & 638 & 35 & 21.3 & 94.8 & 77.3 & 59.1 \\
\hline & Yes & 441 & 119 & & & & \\
\hline
\end{tabular}

Abbreviations: NPV, negative predictive value; PPV, positive predictive value. Based on the McNemar test for evaluation of classifiers, the improvement (especially in specificity) generated by ILI case definition excluding alternate diagnoses was statistically significant $\left(\chi^{2}=159, P<0.05\right)$.

\section{Results}

Of the 1233 tested for H1N1, only $62.7 \%(n=773)$ had signs and symptoms consistent with ILI, before considering alternate diagnoses. This significantly decreased to $45.4 \%$ $(n=560)\left(\chi^{2}=74, P<0.001\right)$ after extraction of cases with alternate diagnoses.

\section{Conclusions}

By excluding patients with alternate diagnoses, ILI case definition specificity was improved, without significant difference in other indices. Dual diagnoses of H1N1 and pneumonia were present in 15 patients, which complicated ILI status designation. In this study, these cases were considered ILI negative because of their alternate diagnoses. In addition, there was a $17.3 \%$ decrease in the overall ILI prevalence rate by excluding cases with an alternate diagnosis. This demonstrates how surveillance methodology may affect ILI rates reported by hospitals, and subsequently

This is an Open Access article distributed under the terms of the Creative Commons Attribution licence (http://creativecommons.org/licenses/by/2.5) OPEN - ACCESS which permits unrestricted use, distribution, and reproduction in any medium, provided the original work is properly cited. 
may affect regional public health surveillance data, necessary for appropriate response.

Accurately identifying ILI patients with alternate diagnoses can be difficult because of lack of available data, specifically lab results, which can be delayed up to $48 \mathrm{~h}$. By using GUARDIAN in this study, we were able to automatically and accurately identify ILI patients with other known causes, and increase our accuracy in identifying H1N1 cases. This study exemplifies the importance of an accurate and consistent clinical case definition for the diagnosis of $\mathrm{H} 1 \mathrm{~N} 1$, along with an automated real-time surveillance system.

\section{Acknowledgements}

This paper was presented as an oral presentation at the 2010 International Society for Disease Surveillance Conference, held in Park City, UT, USA, on 1-2 December 2010.

\section{References}

1 Centers for Disease Control and Prevention. http://www.cdc.gov/.

2 Waddell MJ, Doseck SM, Silva JC, Rumoro DP. GUARDIAN: Geographic Utilization of Artificial intelligence in Real-Time for Disease Identification and Notification. Adv in Dis Surveill 2007;4:63. 


\title{
Access to and use of syndromic surveillance information at the local health department level
}

\author{
E Samoff ${ }^{1}$, A Ising ${ }^{1,2}$, A Waller ${ }^{1,2}$, E Sickbert-Bennett ${ }^{3}$, M Davis ${ }^{1}$, M Park ${ }^{1}$, SW Haas ${ }^{4}$, L DiBiase ${ }^{1}$, and PDM MacDonald ${ }^{1,5}$ \\ ${ }^{1}$ North Carolina Preparedness and Emergency Response Research Center, Gillings School of Global Public Health, University of North Carolina \\ at Chapel Hill (UNC-CH), Chapel Hill, NC, USA; ${ }^{2}$ Carolina Center for Health Informatics, Department of Emergency Medicine, UNC-CH, Chapel \\ Hill, NC, USA; ${ }^{3}$ North Carolina Division of Public Health, Chapel Hill, NC, USA; ${ }^{4}$ School for Information and Library Science, UNC-CH, \\ Chapel Hill, NC, USA; and ${ }^{5}$ Department of Epidemiology, UNC-CH, Chapel Hill, NC, USA \\ E-mail: erika.samoff@unc.edu
}

\section{Objective}

To characterize use of syndromic surveillance information for key public health functions at the local health department level, and to make recommendations to facilitate use of syndromic surveillance data for these functions.

\section{Introduction}

Syndromic surveillance data have been widely shown to be useful to large health departments. Use at smaller local health departments (LHDs) has rarely been described, and the effectiveness of various methods of delivering syndromic surveillance data and information to smaller health departments is unknown. Syndromic surveillance data and information in North Carolina are available to all local public health staff by several routes. This report characterizes LHD access to syndromic surveillance data and information and their use for key public health purposes.

\section{Methods}

Structured interviews were conducted with local health directors and communicable disease nursing staff from a stratified random sample of LHDs during May through September 2009. The survey captured information on the use of the North Carolina Disease Event Tracking and Epidemiologic Collection Tool (NC DETECT) web application for direct data access and on the use of syndromic surveillance information for outbreak management, program management, and the creation of reports. Data on direct access to NC DETECT were available for all 85 LHDs in the state.

\section{Results}

Surveys were completed with 14 of 15 LHDs (93\% response rate). Syndromic surveillance data were used by LHDs for outbreak management (two of $14(15 \%)$ ), program management (three of $14(21 \%)$ ), and creating reports for distribution to public health stakeholders and partners (four of $14(26 \%))$.

LHD staff obtained syndromic surveillance information from hospital-based public health epidemiologists (PHEs) and public health regional surveillance teams (PHRSTs), and syndromic surveillance data directly from NC DETECT. The information distributed by PHEs includes both reports and notification of specific events by telephone, and may include relevant information from patient-hospital records; 22 of 85 LHDs (26\%) had access to a PHE. The information distributed by PHRSTs includes regularly distributed standardized reports. Direct access to NC DETECT allows LHDs to create and review aggregate data and to review single event details; direct access is voluntary. At the time of this survey, 29 of 85 LHDs (34\%) had NC DETECT logins; staff at 10 of 85 LHDs (34\%) accessed the system six or more times in the 6 months before the survey. Use of syndromic surveillance information for outbreak, program, and report uses was associated with receiving information from PHEs or PHRSTs.

Reasons given for not using syndromic surveillance information focused on the time or effort needed to obtain useful information from large NC DETECT data sets. Information from this survey and other North Carolina sources was gathered to support the design of an LHD web portal for NC DETECT. A draft version of this portal that attempts to automate some of the filtering role provided by PHEs and PHRSTs, will be presented.

\section{Conclusions}

Syndromic surveillance information is useful to the local level, as evidenced by use of syndromic surveillance information for outbreak, program, and report purposes. Syndromic surveillance information and data are available to

This is an Open Access article distributed under the terms of the Creative Commons Attribution licence (http://creativecommons.org/licenses/by/2.5) OPEN - access which permits unrestricted use, distribution, and reproduction in any medium, provided the original work is properly cited. 
LHDs, and some LHD staff members directly access NC DETECT. However, functional use of this information for public health purposes occurs only where these data have been filtered and reviewed by public health personnel who are routine users of NC DETECT. These results show that distribution of syndromic surveillance information by state and regional staff is effective, and suggest that improvements that support more efficient filtering and interpretation of syndromic surveillance data, as well as provision of training, may increase use of this information at the local level.

\section{Acknowledgements}

This paper was presented as a poster at the 2010 International Society for Disease Surveillance Conference, held in Park City, UT, USA, on 1-2 December 2010. 


\title{
Tracking H1N1 vaccine doses administered using CRA system
}

\author{
B Nichols ${ }^{1}$, S Waite ${ }^{2}$, H Hill ${ }^{3}$, L Han-Lee ${ }^{2}$, S Sapkota ${ }^{2}$, and J Tropper ${ }^{1}$ \\ ${ }^{1}$ Centers for Disease Control and Prevention (CDC), Atlanta, GA, USA; ${ }^{2}$ CDC Contractor from SRA International Inc., Fairfax, VA, USA; and \\ ${ }^{3}$ CDC Contractor from McKing Consulting, Fairfax, VA, USA \\ E-mail: ssapkota@cdc.gov
}

\section{Objective}

The objective of this paper is to report the use of the Centers for Disease Control and Prevention's (CDC) Countermeasure and Response Administration System (CRA) to track and monitor H1N1 doses administered during the initial weeks of the 2009-2010 H1N1 Vaccine Program when supplies of the vaccines were limited, and before population-based surveys like Behavioral Risk Factor Surveillance Systems, and National H1N1 Flu Survey could effectively monitor vaccine coverage. ${ }^{1}$

\section{Introduction}

The novel strain of H1N1 Influenza A virus, which first caused localized outbreaks in parts of Mexico, was declared a pandemic in June 2009. CDC's CRA was used to track the H1N1 vaccine uptake across population age groups during the first eight weeks of the event (3 October to 21 November 2009). The CRA application was utilized to track vaccine doses administered in the initial period of H1N1 vaccine campaign, as there was no other method available to inform how well the vaccine was reaching target age groups.

\section{Methods}

Through several webinars and conference calls with vaccinetracking coordinators, Project Areas were required to participate in reporting $\mathrm{H} 1 \mathrm{~N} 1$ vaccine doses-administered counts on a weekly basis. All 50 states, four major cities (Los Angeles, New York City, Chicago and Washington DC) and eight US islands and territories, also known as Project Areas, participated in the campaign. Reporting occurred along seven Advisory Committee on Immunization Practices (ACIP) recommended age groups: 6-23 months; 24-59 months; 5-18 years; $19-24$ years; 25-49 years; 50-64 years; and 65 years and above. Each Project Area was asked to select an option from the following to transmit report/data to CDC: Option 1, data exchange; Option 2 , web entry aggregate; Option 3 , web entry detail.

\section{Results}

During the initial eight-week-period of the H1N1 vaccine response, from 3 October to 21 November 2009, a total of
$14,788,795$ vaccines were reported to CDC from 60 reporting Project Areas. Of the total doses reported, 35\% of vaccines were administered to children aged 5-18 years, and over $83 \%$ were administered to persons less than 65 years. The average delay between administration of H1N1 vaccine and reporting of that data to CRA was from two to four weeks. Overall, by the final week of reporting (21 November 2009), Project Areas reported approximately $4.4 \%$ of H1N1 vaccine administered during the first eight weeks of the campaign using the CRA system.

\section{Conclusions}

CRA is a functioning informatics system for monitoring vaccine uptake in different age groups in the early stages of the H1N1 response when vaccine supply is inadequate. This vaccine reporting event demonstrated that CRA application is a useful system for national reporting of aggregate doses administered early on during a pandemic, providing an understanding of vaccine coverage before the traditional surveillance systems. Comparisons of data obtained from NHFS validated data from CRA application. ${ }^{2}$ Improvement on individual Project Area automation would contribute significantly towards enhancing doses-administered reporting systems.

\section{Acknowledgements}

This paper was presented as a poster at the 2010 International Society for Disease Surveillance Conference, held in Park City, UT, USA, on 1-2 December 2010.

\section{References}

1 Tropper J, Shimabukuro T, Sapkota S, Williams W, Williams CE, Stanley T. CDC's Countermeasure and Response Administration system for monitoring $\mathrm{H} 1 \mathrm{~N} 1$ vaccine doses administered. Disaster Med Public Health Prep 2009;2 (Suppl): S107-8.

2 CDC. Interim Results: Influenza A (H1N1) 2009 Monovalent Vaccination Coverage-United States, October-December 2009. MMWR 2010;59(02):44-8. This is an Open Access article distributed under the terms of the Creative Commons Attribution licence (http://creativecommons.org/licenses/by/2.5)
open which permits unrestricted use, distribution, and reproduction in any medium, provided the original work is properly cited. 


\title{
User requirements for a user-centered design (UCD) redesign of a public health surveillance system: BioSense
}

\author{
REN Sembajwe, TR Farris, OL Rojas-Smith, BL Massoudi, AK Banger, and A Casoglos
}

RTI International, Research Triangle Park, NC, USA

E-mail: rsembajwe@rti.org

\section{Objective}

The objective of this poster presentation is to provide information on the efforts and results of the user requirements gathering process, pursued in the redesign of the BioSense system employing a User-Centered Design (UCD) approach. A UCD system development approach studies the people that the system will serve, and involves them through the planning, design, and development processes.

\section{Introduction}

The Centers for Disease Control and Prevention (CDC) launched the BioSense program in 2003 to establish an integrated system of nationwide public health surveillance for the early detection and assessment of potential bioterrorism-related illness. Over the ensuing years, the original aims of BioSense were broadened to meet evolving public health surveillance needs and priorities.

In 2009, CDC embarked on a redesign of the BioSense system that would retain the original purpose of early event (or threat) detection and characterization, but enhance the capacity for situation awareness, event response, and routine public health practice. The BioSense redesign will be accomplished through continuous involvement of state and local users, and technical experts in the planning, design, development, and testing phases of the new BioSense system.

\section{Methods}

Information regarding state and local biosurveillance practices was gathered from end users and other stakeholders through user sessions convened at conferences, workshops, and ad-hoc meetings throughout 2009 and 2010. Participants in user sessions were primarily state and local health department stakeholders. Information on biosurveillance practices was also gathered from General Accounting Office (GAO) reports, 2009 BioSense evaluations, and Technical Expert Panel feedback. The information was grouped, analyzed, and weighted/ranked on the basis of.frequency, and then prioritized and translated into meaningful user requirements for the system redesign.

\section{Results}

The user requirements analysis identified challenges and suggestions in five key areas: ${ }^{1}$ capacity (workforce presence, skill set, and funding resources), ${ }^{2}$ governance (stakeholder engagement, design, and purpose of system), ${ }^{3}$ policy (regulations and legislation), ${ }^{4}$ standards and definitions (taxonomies, business rules, and case definitions), ${ }^{5}$ data (sources, quality, and access), technological infrastructure, and application design (functionality and usability).

\section{Conclusions}

The user requirements gathering process identified gaps in biosurveillance practices and systems that BioSense can directly address in the redesign, which will result in more effective and timely public health surveillance at the local, state, and national levels.

\section{Acknowledgements}

This paper was presented as a poster at the 2010 International Society for Disease Surveillance Conference, held in Park City, UT, USA, on 1-2 December 2010.

\section{References}

1 Buehler JW, Whitney EA, Smith D, Prietula MJ, Stanton SH, Isakov AP. Situational uses of syndromic surveillance. Biosecur Bioterror 2009;2:166-77.

2 Lesesne DB, Leese PJ, Rojas-Smith OL, Rein DB. Characteristics and geographic dispersion of syndromic surveillance in the United States in 2008, 2009. Unpublished manuscript.

3 Uscher-Pines 1, Farrell CL, Cattani J, Hsieh YH, Moskal MD, Babin SM. A survey of usage protocols of syndromic surveillance

OPenss is an Open Access article distributed under the terms of the Creative Commons Attribution licence (http://creativecommons.org/licenses/by/2.5) OPEN - ACCESS which permits unrestricted use, distribution, and reproduction in any medium, provided the original work is properly cited. 
systems by state public health departments in the United States. J Public Health Manag Pract 2009;5:432-8.

4 Usability Professionals' Association, Promoting usability concepts and techniques worldwide: What is user-centered design? Available at: http://www.upassoc.org/usability_resources/about usability/what_is_ucd.html. Accessed September 1, 2010.

5 U.S. General Accounting Office. Health information technology: More detailed plans needed for the Centers for Disease Control and Prevention's redesigned BioSense program. GAO-09-100. Washington, DC: U.S. General Accounting Office, 2008. Available at: http://www.gao.gov. Accessed August 16, 2010.

6 U.S. General Accounting Office. Information technology: Federal agencies face challenges in implementing initiatives to improve public health infrastructure. GAO-05-308. Washington, DC: U.S. General Accounting Office, 2005. Available at: http://www.gao. gov. Accessed August 16, 2010. 


\title{
Using Twitter to estimate H1N1 influenza activity
}

\author{
A Signorini ${ }^{1}$, PM Polgreen ${ }^{2}$, and AM Segre ${ }^{1}$ \\ ${ }^{1}$ Departments of Computer Science, The University of lowa, lowa City, IA, USA; and ${ }^{2}$ Internal Medicine, The University of lowa, lowa City, IA, USA \\ E-mail: alessio-signorini@uiowa.edu
}

\section{Objective}

This paper describes a system that uses Twitter to estimate influenza-like illness levels by geographic region.

\section{Introduction}

Twitter is a free social networking and micro-blogging service that enables its millions of users to send and read each other's 'tweets', or short messages limited to 140 characters. The service has more than 190 million registered users and processes about 55 million tweets per day. ${ }^{1}$ Despite a high level of chatter, the Twitter stream does contain useful information, and, because tweets are often sent from handheld platforms on location, they convey more immediacy than other social networking systems.

\section{Methods}

We collected and stored all public tweets beginning October 1, 2009 that matched a set of pre-specified search terms (for example, flu, swine, influenza, tamiflu, oseltamivir, H1N1, and so on). After culling, for example, non-US and non-English tweets, and applying appropriate stemming algorithms, the remaining tweets were used to produce a dictionary of English word equivalents. We compiled daily and weekly usage statistics for each dictionary term, both nationally and at the CDC's influenza reporting-region level. ${ }^{2}$ These weekly term-usage statistics were then used to estimate weekly levels of influenzalike illness (ILI). Using Support Vector Regression, ${ }^{3}$ a supervised machine-learning method generally applied to solve classification problems, ${ }^{4}$ we trained our system using weekly termfrequency statistics from tweets issued outside of CDC Region two (New York and New Jersey) and ILI values reported by the CDC for the weeks October 4-10, 2009 through May 16-22, 2010. We then used the resulting system to estimate ILI in CDC Region two, thus performing an out-of-sample validation.

\section{Results}

The Figure 1 shows estimated weighted ILI values for CDC Region two (New Jersey and New York) produced by our system when trained on Twitter traffic exclusive of CDC Region two. The predicted weekly ILI values are shown in red, with ILI values reported later by the CDC in green. Our regional model approximates the epidemic curve reported by ILI data with an average error of $0.37 \% \quad(\mathrm{~min}=0.01 \%$, $\max =1.25 \%$, and a standard deviation of $0.26 \%$. Similar results were obtained when estimating ILI at a national level.

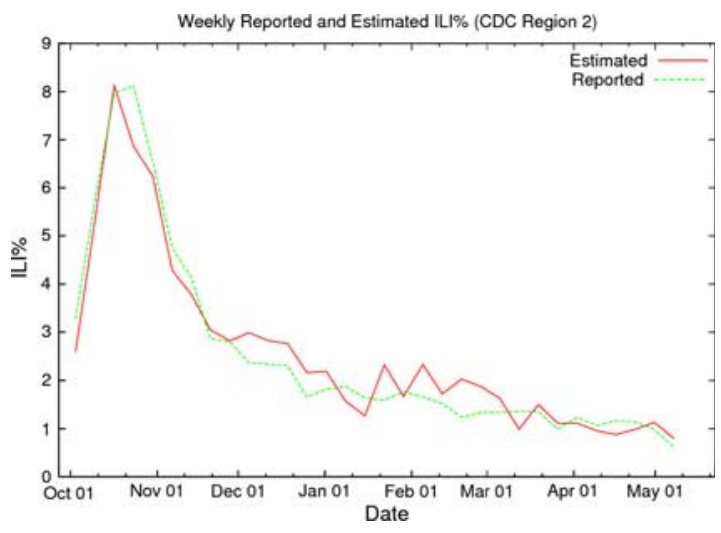

\section{Conclusions}

Our results demonstrate that Twitter traffic can be used to provide real-time estimates of disease activity. Our ability to quickly detect trends, which are then confirmed by observations from traditional surveillance approaches, make this new form of surveillance a promising area of research at the interface between computer science, epidemiology, and medicine.

\section{Acknowledgements}

This paper was presented as an oral presentation at the 2010 International Society for Disease Surveillance Conference, held in Park City, UT, USA, on 1-2 December 2010.

\section{References}

1 Lorica B. Twitter by the numbers. O'Reilly Radar, http://radar. oreilly.com/2010/04/twitter-by-the-numbers.html, 14 April 2010 (accessed 15 August 2010).

2 Centers for Disease Control and Prevention. Overview of Influenza Surveillance in the United States, http://www.cdc.gov/flu/weekly/ fluactivity.htm (accessed 15 August 2010).

3 Drucker H, Burges CJC, Kaufman L, Smola A, Vapnik V (1997) 'Support Vector Regression Machines'. Advances in Neural Information Processing Systems 9, NIPS. MIT Press: Cambridge, MA, USA, 1996, pp 155-61.

4 Cristianini N, Shawe-Taylor J. An Introduction to Support Vector Machines and Other Kernel-based Learning Methods. Cambridge University Press: Cambridge, England, 2000.

open -2 access This is an Open Access article distributed under the terms of the Creative Commons Attribution licence (http://creativecommons.org/licenses/by/2.5) OPEN - ACCESS which permits unrestricted use, distribution, and reproduction in any medium, provided the original work is properly cited. 


\title{
How did specific specialties and new sentinel providers affect ILINet data post 2009 A/H1N1 recognition
}

\author{
O Simwale
}

Pennsylvania Department of Health, Bureau of Epidemiology, Division of Infectious Disease Epidemiology, Harrisburg, PA, USA E-mail: owsimwale@state.pa.us

\section{Objective}

The objective of this study is to describe changes in influenza-like illness (ILI) surveillance, eight weeks before and after the 2009 A/H1N1 pandemic influenza outbreak. We examined changes in provider recruitment, composition, reporting of ILI, and we characterize ILI data in terms of timeliness, and ILI baselines by type of sentinel provider.

\section{Introduction}

The United States outpatient Influenza-like Illness Surveillance Network (ILINet) is one of the five systems used for influenza surveillance in the United States. In Pennsylvania, ILINet providers are asked to report, every Monday, the total number of patients seen for any cause, and the number of patients with influenza-like illness (ILI) by age group. In order to encourage timely reporting, weekly reminders along with a data summary were sent to all sentinel providers postoutbreak recognition. Through the study period, recruitment of new sentinel sites was done through local health departments, health alerts, and training sessions. Sentinel providers were not restricted from submitting specimens to the state lab before and after the outbreak, whereas non sentinel providers had strict restrictions.

\section{Methods}

We examined ILINet data pre-outbreak recognition (January to March 2009) and post-outbreak recognition (April to June 2009) changes in provider recruitment, composition, reporting of ILI, and we characterized ILI data in terms of timeliness, and computed ILI baselines by type of sentinel provider. In this study, we defined timeliness as the number of providers reporting by Close of Business every Monday.

\section{Results}

In multivariate analyses of preliminary data, and after controlling for observed covariates, we noted a two-fold increase in number of sentinel providers $(60-136, P>0.05)$. Whereas before the outbreak the composition of sentinel providers was limited to pediatricians and family practices physicians, a variety of practices including Obstetricians and Gynecologists, colleges, and emergency rooms joined the sentinel providers post-outbreak recognition. Sentinel colleges reported significantly higher levels of ILI, pediatricians, emergency rooms, and family practice offices (8 versus 3 versus $2 \%$ ). There was a significant increase in the proportion of sentinel providers reporting ILI every week postoutbreak recognition (50 versus $20 \%, P>0.5$ ). There was a week correlation between the numbers of sentinel providers reporting every week and the proportion of ILI visits reported $(r=0.05)$. We also noted a significant difference between the levels of ILI reported every Monday compared with what is reported by Thursday of each week $(P>0.5)$.

\section{Conclusion}

Our study highlights several changes in ILI surveillance post A/H1N1 recognition. The number and composition of sentinel providers increased significantly post-outbreak recognition. Timely reporting of ILI should be encouraged in order to allow for near-real time use of ILINet data. An ILINet data should be analyzed at provider level in order to establish provider-specific and region-specific ILI baselines.

\section{Acknowledgements}

This paper was presented as a poster at the 2010 International Society for Disease Surveillance Conference, held in Park City, UT, USA, on 1-2 December 2010.

open Access This is an Open Access article distributed under the terms of the Creative Commons Attribution licence (http://creativecommons.org/licenses/by/2.5) OPEN - ACCESS which permits unrestricted use, distribution, and reproduction in any medium, provided the original work is properly cited. 


\title{
Use of a public health working group to coordinate multi-jurisdiction response to bioterrorism surveillance signals and influenza outbreaks
}

\author{
AJ Siniscalchi \\ State of Connecticut, Department of Public Health, Hartford, CT, USA \\ E-mail: alan.siniscalchi@ct.gov
}

\begin{abstract}
Objective
The rapid and effective coordination of the multi-jurisdictional communications and response to a surveillance system signal are an important goal of public health preparedness planning. This goal is particularly challenging if the signal indicates a possible risk that could adversely affect populations in multiple states and municipalities. This paper examines the value of a regional workgroup in the activation, integration, and coordination of multiple surveillance systems along with efforts to coordinate risk communication messaging. Recommendations for the development of similar groups in other regions are discussed.
\end{abstract}

\section{Introduction}

The use of syndromic surveillance systems to detect illness and outbreaks in the mid 1990s in New York City resulted in recommendations for increased use of these systems for detection of bioterrorist agents, and tracking influenza throughout the region. ${ }^{1,2}$ Discussions on approaches to best respond to surveillance system signals led to initial efforts to organize a coordinating group of various public health agencies throughout the New York City region. These efforts were strengthened after the events of September 11, 2001, and resulted in the development of a regional workgroup consisting of epidemiologists and other staff from all state, county, and municipal health departments who operate, respond to, or oversee public health preparedness surveillance systems throughout the greater New York City metropolitan area.

\section{Methods}

Syndromic surveillance system data from hospital-based networks in state and large municipal public health jurisdictions are reviewed on a daily basis along with information from laboratory-based and remote-sensing systems.
Information on surveillance system signals and other indicators of bioterrorism events or emerging infectious disease outbreaks are shared among members of the workgroup, as appropriate. This information can be used for an enhanced review of syndromic surveillance system data in other jurisdictions, notification of emergency department staff to look for patterns of illness, and recommendations for increased laboratory testing. Findings of laboratoryconfirmed bioterrorist agents and evidence of outbreaks of emerging illness would result in development of coordinated messaging among member jurisdictions of the work group. Periodic meetings and exercises are scheduled among members that include representatives of federal, state, and local law enforcement agencies. Joint public health and law enforcement emergency response protocols have also been developed and tested.

\section{Results}

Members of the work group have coordinated a number of joint multi-jurisdictional surveillance system signal response investigations. These include the regional response to the 2001 anthrax mail attacks (Amerithrax), ${ }^{3}$ the identification of inhalation ${ }^{4}$ and cutaneous ${ }^{5}$ anthrax cases in individuals exposed to contaminated goatskins used in African drums, targeted regional surveillance for detection of influenza and other disease events following the 2009 US Presidential Inauguration $^{6}$ and tracking of the novel 2009 influenza A (H1N1) pandemic. ${ }^{6}$

\section{Conclusions}

The use of a multi-jurisdictional public health working group has resulted in the enhanced coordination of a regional response to various surveillance system signals and emerging disease outbreaks. Development of similar working groups is recommended for other regions.

This is an Open Access article distributed under the terms of the Creative Commons Attribution licence (http://creativecommons.org/licenses/by/2.5) OPEN - ACCESS which permits unrestricted use, distribution, and reproduction in any medium, provided the original work is properly cited. 


\section{Acknowledgements}

This paper was presented as a poster at the 2010 International Society for Disease Surveillance Conference, held in Park City, UT, USA, on 1-2 December 2010.

\section{References}

1 Heffernan R, Mostashari F, Das D, Besculides M, Rodriguez C, Weiss D, et al. System descriptions New York City syndromic surveillance systems. MMWR 2004;53 (Suppl): 23-7.
2 Hadler JL, Siniscalchi A, Dembek Z. Hospital admissions syndromic surveillance: experience monitoring serious rash illness and influenza. MMWR 2005;54 (Suppl): 169-73.

3 CDC. Update: investigation of bioterrorism-related anthrax Connecticut, 2001. MMWR 2001;50:1077-9.

4 CDC. Inhalation anthrax associated with dried animal hides Pennsylvania and New York City, April 2006. MMWR 2006;55:280-2.

5 CDC. Cutaneous anthrax associated with drum making using goat hides from West Africa - Connecticut, 2007. MMWR 2008;57:628-31.

6 Siniscalchi A, Andrews M, Purviance K, Esponda B, Brennan T, Nelson R, et al. Yes we can! use of multiple surveillance systems for detection of post inaguration and late season influenza events, 2009. ISDS Annual Conference. 


\title{
A comparison of syndromic surveillance chief complaint data and discharge data in a pediatric hospital system during 2009 H1N1
}

\author{
D Peace ${ }^{1}$, W Smith ${ }^{2}$, A Reeves ${ }^{2}$, D Little ${ }^{2}$, K Soetebier $^{2}$, and C Drenzek ${ }^{2}$ \\ ${ }^{1}$ Children's Hospital of Atlanta, Atlanta, GA, USA; and ${ }^{2}$ Georgia Division of Public Health, Atlanta, GA, USA \\ E-mail: delittle@dhr.state.ga.us
}

\begin{abstract}
Objective
The objective of this study is to describe the difference between patient chief complaint (CC), influenza- like- illness (ILI) data provided daily to the Georgia Syndromic Surveillance Program (SSP) during the $2009 \mathrm{H} 1 \mathrm{~N} 1$ pandemic, and patient discharge data (DD) subsequently provided for comparison with the SSP from its participating pediatric hospital system, and its two affiliated emergency rooms.
\end{abstract}

\section{Introduction}

The Syndromic Surveillance Program (SSP) of the Acute Disease Epidemiology Section of the Georgia Division of Public Health, provides electronic ILI data to the Center for Disease Control and Prevention's Influenza-like Illness Surveillance Network (ILINet) Program that characterizes the burden of influenza in states on a weekly basis.

ILI is defined as a fever of $100^{\circ}$, plus a cough or sore throat. This definition is used to classify ILI by the SSP, as well as in diagnosis at the pediatric hospital system. During the 2009 H1N1 pandemic, the SSP was provided a daily data transfer to the Center for Disease Control and Prevention to heighten situational awareness of the burden of ILI in Georgia. Throughout the peak of the pandemic, data from the pediatric hospital system identified when the percentage of daily visits for ILI had substantively increased. The data includes patient CC data from emergency department visits for two facilities at Facilities A and B. The data received by SSP does not include diagnosis data.

Patient emergency department DD for 'FLU' was provided to SSP retrospectively to compare with the CC data routinely collected and analyzed. The data was derived from the pediatric health system's month end, internal, syndromic surveillance report based upon emergency department visits, and including physician's diagnosis at the time of patient's discharge. The case definition of 'FLU' from the pediatric health system facilities is acute onset of fever, with cough and/or sore throat in the absence of a known cause other than influenza.

\section{Methods}

The data were evaluated by analyzing the percentage of 'FLU'-DD visits during 2009, in contrast to the percentage of ILI-CC visits provided to SSP daily from Facilities A and B. The total percentage of ILI visits to both facilities for CC and DD were then compared and correlated by Facilities A and B, observing 'FLU'-DD to the SSP-ILI-CC data. The CC data were then assessed for its ability to accurately identify changes in actual influenza activity in the two facilities during corresponding time period using Pearson's correlation coefficient. Finally, CC-ILI data were compared with CDC's National Respiratory and Enteric Virus Surveillance System (NREVSS), and ILINet data for Georgia.

\section{Results}

The differences between ILI-DD and CC, from facilities A and B were substantial. When comparing Facility A and B, ILI data separately or combined, the burden of ILI based on CC was substantively higher than the observed DD for the same time period. Interestingly, patients from Facility A were more likely to receive an influenza diagnosis than patients from Facility B. The case definition between the facilities is the same; the reason for the difference is not clear (Figure 1).

The SSP-ILI-CC data that were compared with Georgia's NREVSS, and ILINet data overestimated the burden of influenza, as expected. However, the SSP-CC-ILI data were effective in reflecting both increases and decreases in influenza activity that were shown in NREVSS and ILINet data.

\section{Conclusions}

The advantages of using electronic ILI-CC data during an evolving event, such as pandemic, are that it is readily available, and allows public health practitioners to characterize the health seeking behaviors of the population. Although it This is an Open Access article distributed under the terms of the Creative Commons Attribution licence (http://creativecommons.org/licenses/by/2.5)
which permits unrestricted use, distribution, and reproduction in any medium, provided the original work is properly cited. 


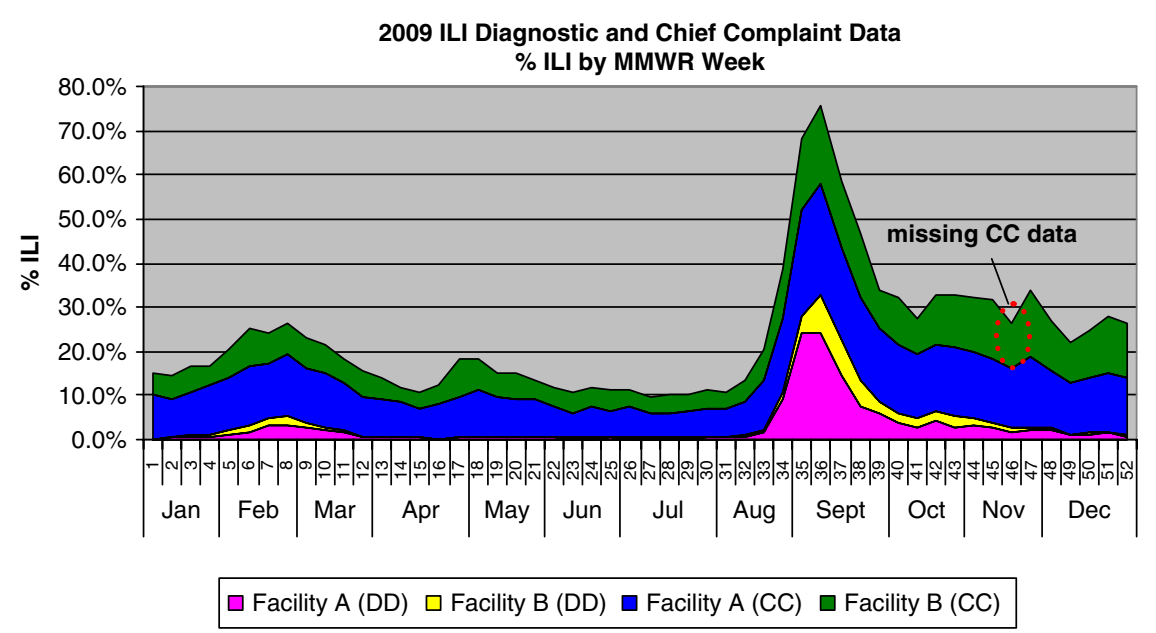

Figure 1 Weekly graph of \% ILI for chief complaint (blue/green), and discharge diagnosis (pink/yellow) visits for facilities A and B in 2009.

still remains difficult to accurately quantify the amount of influenza activity because of the overestimation of ILI disease burden that CC data produces, its ability to reflect trends in the burden of diagnosed influenza in the population is valuable.

\section{Acknowledgements}

This paper was presented as a poster at the 2010 International Society for Disease Surveillance Conference, held in Park City, UT, USA, on 1-2 December 2010. 


\title{
Design and development of a standards-based model to publish public health reporting criteria
}

\author{
C Staes ${ }^{1}$, D Rajeev ${ }^{1}$, R Bradshaw ${ }^{1}$, S Mottice ${ }^{2}$, Z Lui $^{2}$, N Hulse $^{1}$, and S Narus ${ }^{1}$ \\ ${ }^{1}$ Department of Biomedical Informatics, University of Utah, Salt Lake City, UT, USA; and ${ }^{2}$ Utah Department of Health, Salt Lake City, UT, USA \\ E-mail: catherine.staes@hsc.utah.edu
}

\section{Objective}

In this paper, we describe the content and functional requirements for a knowledge management system that can be authored by public health authorities to inform reporting facilities 'what's reportable where'.

\section{Introduction}

State laws mandate clinicians and laboratories to report occurrences of reportable diseases to public health entities. For this purpose, a set of case-reporting specifications are published and maintained by public health departments. There are several problems with the existing case-reporting specifications: (1) they are described on individual state websites and posters and not structured or executable; (2) the specifications are often misleading representing case classification rather than case reporting criteria; (3) they vary across jurisdictions and change over time; and (4) reporting facilities are required to interpret the criteria and maintain logic in their own systems. ${ }^{1}$ To overcome these problems, we are designing and developing a prototype system to represent case-reporting specifications that can be authored and maintained by public health and published openly.

\section{Methods}

To determine the content and functional requirements, we reviewed existing reportable disease lists and rules, fact sheets, and CSTE Position Statements. We used ethnographic methods to obtain feedback from public health authorities. To model the knowledge using standards, we reviewed existing HL7-structured documents including the Clinical Document Architecture. The knowledge management system allows public health authorities to author, store, and publish knowledge-concerning reporting logic and specifications (Figure 1). For this demonstration project, the reporting entities will be able to view human-readable specifications, download structured content using web services for execution within their own systems, and subscribe or query for updates. Knowledge authoring, publication, and access are provided through serviceoriented architecture.

\section{Results}

After review of the websites from Utah, LA County, Colorado and Washington State for communicable disease and environmental/occupational diseases, we identified 103 reportable events, of which only 62 were included among the nationally notifiable list. The reportable events included diagnoses, laboratory results, clinical observations, interventions, outbreaks, and intent. We identified the following content requirements to represent reporting specifications: (a) detection criteria that includes clinical and laboratory findings; (b) constraints including characteristics of the patient, encounter, laboratory or clinical setting; (c) reference resources; and (d) reporting action details including the reporting time frame and so on. We have designed a model workflow for public health authorities to author reporting specifications, allowing for default content based on the CSTE disease specific 'Position Statements'. We are developing the use case for accessing the information in both human-readable and machine-processable format. We are storing the knowledge assets in XML and exploring the HL7structured document format to structure reporting specifica-

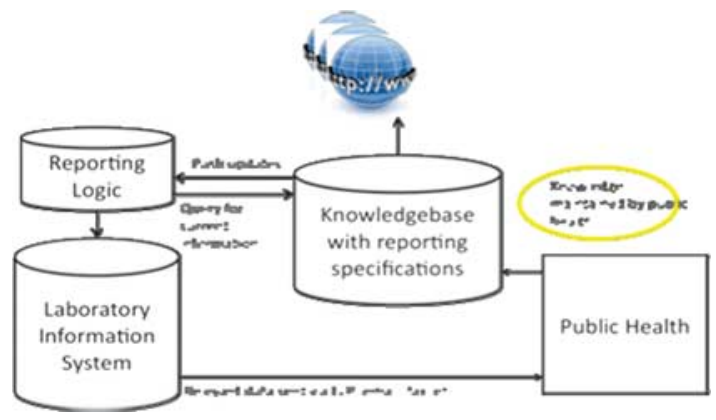

Figure 1 Future process for laboratories to determine 'what is reportable where' and maintain logic.

open 2 Access This is an Open Access article distributed under the terms of the Creative Commons Attribution licence (http://creativecommons.org/licenses/by/2.5) OPEN - access which permits unrestricted use, distribution, and reproduction in any medium, provided the original work is properly cited. 
tions. We are exploring models to represent laboratory criteria for improved linkage to the relevant pairs of LOINC/SNOMED mappings.

\section{Discussion}

We have demonstrated the modeling of a knowledge management system using existing standards. The development of a prototype knowledge management system that allows public health authorities to author, store, and publish knowledge-concerning reporting specifications will help inform the development and implementation of a national system.

\section{Acknowledgements}

This paper was presented as an oral presentation at the 2010 International Society for Disease Surveillance Conference, held in Park City, UT, USA, on 1-2 December 2010. Funding: (Grant\# 1PO1HK000069-01) Rocky Mountain Center for Translational Research in Public Health Informatics, NLM Training Grant \# 5T15LM007124 (DR).

\section{Reference}

1 M'ikanatha NM, Welliver DP, Rohn DD, Julian KG, Lautenbach E. Use of the web by state and territorial health departments to promote reporting of infectious disease. JAMA 2004;291:1069-71. 


\title{
Use of emergency department data for case finding following a community anthrax exposure
}

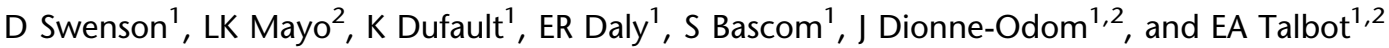 \\ ${ }^{1}$ New Hampshire Department of Health and Humans Services, Concord, NH, USA; and ${ }^{2}$ Dartmouth-Hitchcock Medical Center, Lebanon, \\ $N H, U S A$ \\ E-mail: dswenson@dhhs.state.nh.us
}

\section{Objective}

This paper describes the use of customizable tools to query electronic emergency department data, as part of case finding, during the response to a community anthrax exposure in New Hampshire.

\section{Introduction}

On 24 December 2009, a female New Hampshire resident was confirmed to have gastrointestinal anthrax on the basis of clinical findings and laboratory testing. ${ }^{1}$ Her source of anthrax was not immediately known, so the New Hampshire Department of Health and Human Services (DHHS), in conjunction with several other state and federal agencies, conducted a comprehensive epidemiologic investigation, which included active surveillance to identify any additional anthrax cases from a similar exposure. It was determined that the index patient participated in a drumming event with animal-hide drums on 4 December, one day before the onset of symptoms. Two drums used at the event were later found to be contaminated with Bacillus anthracis.

\section{Methods}

A retrospective review of emergency department encounters between 1 October and 26 December was conducted immediately after confirmation of the index anthrax case to identify possible anthrax cases that had already presented but were not confirmed because of the rarity of clinical anthrax. In addition, active, prospective surveillance for possible cases continued between 26 December and 31 January, 2010. DHHS's statewide emergency department data system's customizable query tools were used to mine data daily for clinical syndrome terms in chief complaint text fields and ICD-9-CM codes associated with the three manifestations of anthrax (inhalation, cutaneous, and gastrointestinal). Chief complaint text and ICD-9-CM query results were reviewed by surveillance staff and a physician using a clinical criteria protocol to decide whether the case warranted follow-up with hospitals to collect additional clinical and laboratory data.

\section{Results}

Of 1,83,973 emergency department encounters between 1 October 2009 and 31 January 2010, 7111 (3.9\%) met either the chief complaint or ICD-9-CM search criteria. Of these $7111,62(0.9 \%)$ warranted collection of additional information based on the clinical criteria protocol. No additional anthrax cases were identified. Three persons were found seeking anthrax post-exposure prophylaxis at an emergency department. Two of these persons were workers involved in the public health response and one person had attended the drumming event and was previously identified during the contact investigation (Figure 1).

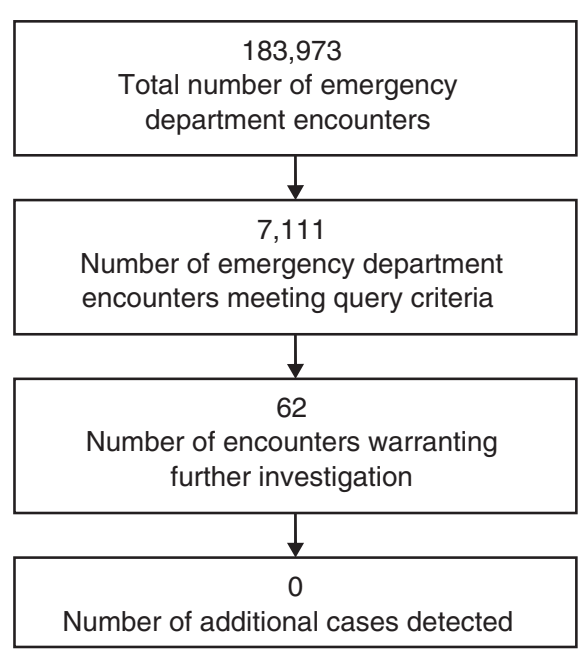

Figure 1 Investigation outcome of emergency department encounters meeting anthrax query and clinical criteria.

open 2 access This is an Open Access article distributed under the terms of the Creative Commons Attribution licence (http://creativecommons.org/licenses/by/2.5) OPEN - access which permits unrestricted use, distribution, and reproduction in any medium, provided the original work is properly cited. 


\section{Conclusions}

New Hampshire's emergency department surveillance system was quickly adapted, through the development of custom queries, to investigate an emergent public health threat. The query tool and clinical criteria protocol allowed for efficient follow-up of only those encounters that were most suspicious for anthrax. These tools narrowed down a pool of nearly 2,00,000 emergency department visits to 62 possible cases requiring further investigation, allowing for more efficient use of health department resources.

\section{Acknowledgements}

This paper was presented as an oral presentation at the 2010 International Society for Disease Surveillance Conference, held in Park City, UT, USA, on 1-2 December 2010.

\section{Reference}

1 CDC. Gastrointestinal anthrax after an animal-hide drumming event-New Hampshire and Massachusetts, 2009. MMWR 2010; 59:872-7. 


\title{
Prospective space-time analysis of the 2007 cryptosporidiosis outbreak, Salt Lake County, Utah
}

\author{
M Taddie ${ }^{1,2}$, and I Yamada ${ }^{1,2}$ \\ ${ }^{1}$ Geography Department, University of Utah, Salt Lake City, UT, USA; and ${ }^{2}$ Center for Spatial Information Science, University of Tokyo, Tokyo, \\ Japan \\ E-mail: mtaddie@earthlink.net
}

\section{Objective}

The objective of this study was to investigate if prospectively applied space-time surveillance could have detected significant, emerging clusters as cryptosporidiosis, cases were reported to the Salt Lake Valley Health Department during the 2007 outbreak.

\section{Introduction}

Cryptosporidiosis is a gastrointestinal illness due to a protozoan parasite that is highly contagious, and resistant to multiple disinfectants. ${ }^{1}$ Utah experienced a large, community-wide outbreak of cryptosporidiosis between June and December of $2007 . .^{2,3}$ During this time period, the Utah Department of Health received reports of 1,902 laboratoryconfirmed cryptosporidiosis cases across the state. ${ }^{2}$ Nearly $40 \%$ of these cases occurred in Salt Lake County (SL County), Utah. In past years, SL County averaged fewer than five cases annually; ${ }^{2}$ however, the incidence rate in the county for this entire outbreak was 125.9 per 100,000 person-years. ${ }^{3}$

\section{Methods}

This study utilized a space-time scan statistic implemented in $\operatorname{SaTScan}^{4}$ to test for the occurrence and location(s) of cryptosporidiosis clusters using time-periodic prospective surveillance, and a Poisson probability model. The study area included the jurisdictional purview of the Salt Lake Valley Health Department, which is SL County. Although true disease outbreaks should be characterized by the onset date of each case, onset dates are not collected until after an investigation is started, and the date of the case reported is generally the first piece of case-specific information available to many health departments. Therefore, report dates were used in the prospective space-time analysis to replicate the realistic surveillance processes that occur in health departments, and to mimic a near real-time surveillance system.

\section{Results}

The first cluster signaled approximately 20 days after the first reported case in SL County. This cluster occurred two days before a statewide press release was issued, and 21 days before the implementation of major intervention measures. From August through mid-September, many significant clusters were detected throughout SL County (Figure 1).

\section{Conclusions}

The results of this study suggest that there were distinct spatial-temporal patterns throughout the outbreak period. Therefore, space-time analysis would have been a valuable and complementary tool to temporal surveillance because it could have detected spatial clusters and high-risk areas of disease as they were reported, or emerged. In addition, it may have been useful for targeting intervention strategies and prioritizing investigations during this large community-wide outbreak.

\section{Acknowledgements}

This paper was presented as a poster at the 2010 International Society for Disease Surveillance Conference, held in Park City, UT, USA, on 1-2 December 2010.

\section{References}

1 American Public Health Association. [Cryptosporidiosis]. In Heymann DL (ed). Control of Communicable Diseases Manual, 18th edn. APHA: Washington, DC, 2004, pp 138-41.

2 Utah Department of Health, Bureau of Epidemiology. Report of Investigation: Cryptosporidium Outbreak Linked to Multiple Recreational Water Venues: Utah, 2007. UDOH: Salt Lake County, UT, 2008.

3 Salt Lake Valley Health Department, Bureau of Epidemiology. 2007 Cryptosporidiosis Outbreak Report. SLVHD: Salt Lake County, UT, 2008.

4 Kulldorff M. Information Management Services, Inc: SaTScan (TM) v7.0.3: Software for the spatial and space-time scan statistics. National Cancer Institute: Bethesda, MD, 2007, www.satscan.org.

open 2 access This is an Open Access article distributed under the terms of the Creative Commons Attribution licence (http://creativecommons.org/licenses/by/2.5) OPEN - access which permits unrestricted use, distribution, and reproduction in any medium, provided the original work is properly cited. 

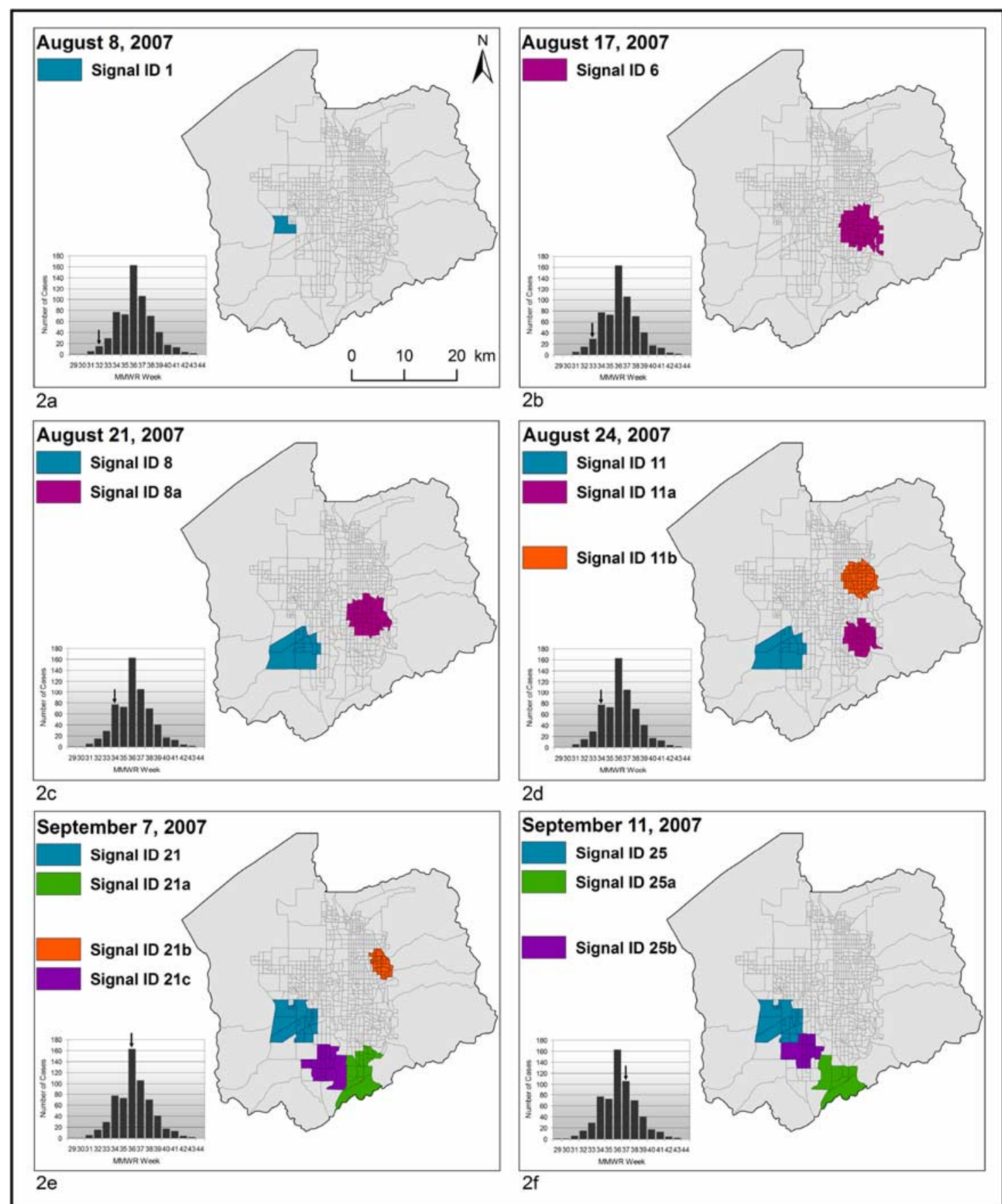

Figure 1 Significant space-time clusters detected during six 28-day time steps from 8 August 2007 to 11 September 2007. 


\title{
An automated influenza-like-illness reporting system using freetext emergency department reports
}

\author{
F-C Tsui ${ }^{1}$, H Su${ }^{1}$, J Dowling ${ }^{1}$, RE Voorhees ${ }^{2}$, JU Espino ${ }^{1}$, and M Wagner ${ }^{1}$ \\ ${ }^{1}$ RODS Laboratory, Department of Biomedical Informatics, University of Pittsburgh, Pittsburgh, PA, USA; and ${ }^{2}$ Office of Epidemiology and \\ Biostatistics, Allegheny County Health Department, Pittsburgh, PA, USA \\ E-mail: tsui2@pitt.edu
}

\section{Objective}

This paper describes an automated Influenza-Like-Illness (ILI) reporting system based on natural language processing of transcribed ED notes and its impact on public health practice at the Allegheny County Health Department (ACHD).

\section{Introduction}

Current methods for influenza surveillance include laboratory confirmed case reporting, sentinel physician reporting of ILI and chief-complaint monitoring from emergency departments (EDs).

The current methods for monitoring influenza have drawbacks. Testing for the presence of the influenza virus is costly and delayed. Specific, sentinel physician reporting is subject to incomplete, delayed reporting. Chief complaint (CC) based surveillance is limited in that a patient's chief complaint will not contain all signs and symptoms of a patient.

A possible solution to the cost, delays, incompleteness and low specificity (for CC) in current methods of influenza surveillance is automated surveillance of ILI using clinicianprovided free-text ED reports.

\section{Methods}

Our ILI reporting system has five parts: an HL7 message parser, the MedLEE natural language processing algorithm, a database, rule-based logic, chart engine and email server. The HL7 message parser extracts ED reports from HL7 messages. Then, MedLEE ${ }^{1}$ finds medical terms contained in each report, including significant negative findings. We store the NLP results in a database using an entity attribute value schema. The rule-based case definitions represent CDC's ILI case definition, (Fever or Chills) and (Cough or Sore throat), ${ }^{2}$ and the symptoms found by the NLP for each patient are tested against this logic. The system plots a 6-month time series graph of the percentage of total ED visits per day with ILI detected by the rule-based logic. The graph also includes a 5-day moving average (centered on the 3rd day) of the daily ILI time series to follow existing practices at ACHD. We employ Jfreechart to create the chart with the two time series. Every day the system sends the report to ACHD using a mail server.

We performed a preliminary evaluation of the system comparing ILI rule-based logic output with manual review of the transcribed ED reports by a physician board, certified in infectious diseases.

\section{Results}

We deployed the ILI reporting system in early May 2009 in Allegheny County. It receives transcribed ED reports by HL7 messages from the seven EDs of the UPMC Health System. The average number of daily $\mathrm{ED}$ visits with $\mathrm{ED}$ reports between July and Dec. 2009 was 569 . Figure 1 shows the ILI chart for 31 December 2009 report that comprises daily ILI percentage and its 5-day moving average between 15 June 2009 and 30 December 2009.

Our evaluation using 140 randomly selected reports (78 positives) found a sensitivity of 94.9\% (95\%CI: 87.54 $97.99 \%$ ), a specificity of $100 \%$ (95\% CI: 94.17-100\%), and a positive predictive value of 100\% (95\% CI: 94.17-100\%).

These charts had three impacts on practice at ACHD: (1) ACHD had daily updates instead of weekly reports (from sentinel physicians). (2) ACHD provided the charts to local media on a regular basis. ${ }^{3}$ (3) ACHD reduced staff time as they no longer had to manually compile ILI reports from sentinel ILI reports (2 days of work for each weekly report).

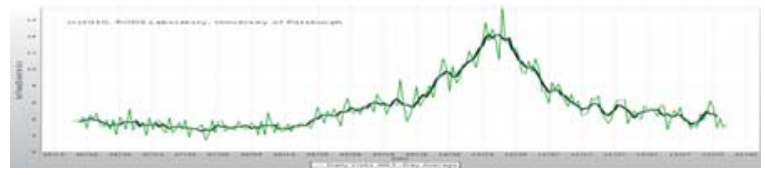

Figure 1 Daily reporting chart showing daily percentage of ED visits with ILI (green) and its 5-day moving average (black) between 15 June 2009 and 30 December 2009. This is an Open Access article distributed under the terms of the Creative Commons Attribution licence (http://creativecommons.org/licenses/by/2.5)
which permits unrestricted use, distribution, and reproduction in any medium, provided the original work is properly cited. 


\section{Conclusions}

We demonstrate utility and method of using free text ED reports for ILI reporting and flu surveillance.

\section{Acknowledgements}

This paper was presented as a poster at the 2010 International Society for Disease Surveillance Conference, held in Park City, UT, USA, on 1-2 December 2010. The project is funded by CDC Grants P01 HK000086 and 1U38 HK000063-01.

\section{References}

1 Friedman C, Shagina L, Lussier Y, Hripcsak G. Automated encoding of clinical documents based on natural language processing. J Am Med Inform Assoc 2004;11:392-402.

2 Bodnar UR, Fielding KL, Navin AW, Maloney SA, Centron MS, Bridges CB, et al. Preliminary Guidelines for the Prevention and Control of Influenza-like Illness among Passengers and Crew Members on Cruise Ships. Center for Disease Control and Prevention, Atlanta, GA, 1999.

3 Two more people die from H1N1 in Pittsburgh area [online] 2009 cited. Available from: http://kdka.com/health/H1N1.flu.deaths. 2.1321133.html. 


\title{
The United States Department of Veterans Affairs
} Integrated Operations Center (VA IOC): collaborations for surveillance, analysis, and prediction for infectious disease threat preparedness - pilot review of dengue occurrence

\author{
KS Wallace ${ }^{1}$, GA Roselle ${ }^{2,3,4}$, S Gamage ${ }^{2}$, D Green ${ }^{5}$, LA Simbart| ${ }^{2}$, M Villeneuve ${ }^{1}$, J Lacey ${ }^{1}$, SM Kralovic ${ }^{2,3,4}$, and JD Riojas ${ }^{1}$ \\ ${ }^{1}$ Office of Operations, Security and Preparedness (OSP), VA Central Office, Washington, DC, USA; ${ }^{2}$ National Infectious Diseases Program \\ Office (IDPO), Veterans Health Administration (VHA), Washington, DC, USA; ${ }^{3}$ Cincinnati VA Medical Center, Cincinnati, OH, USA; ${ }^{4}$ University \\ of Cincinnati College of Medicine, Cincinnati, OH, USA; and ${ }^{5}$ Office of Climate, Water, and Weather Services, National Weather Service (NWS), \\ National Oceanic and Atmospheric Administration (NOAA), Silver Spring, MD, USA \\ E-mail: katherine.wallace@va.gov
}

\section{Objective}

The objective of this study is to describe Veterans Affairs Integrated Operations Center-enabled collaborations to enhance the synergy of relevant data/information from Veterans Affairs (VA) and non-VA partners for improved early warning, and situational awareness of infectious disease threats.

\section{Introduction}

Under leadership of the Secretary of Veterans Affairs (VA), Office of Operations, Security and Preparedness has established the Veterans Affairs Integrated Operations Center, with the goal of enhancing integration and analysis of data, and information from VA's preparedness partners, both internal and external, for timely decision support.

The Office of Operations, Security and Preparedness oversee emergency preparedness for the VA, which includes responsibility for preparedness activities at Veterans Health Administration (VHA). The VHA provides medical care to over 5 million patients a year at 153 medical centers, and over 900 outpatient clinics in the United States, and the United States territories. The Office of Operations, Security and Preparedness is developing a VA-Subject Matter Expertise Center for Biological Events in collaboration with the VHA-National Infectious Diseases Program Office. The Subject Matter Expertise Center for Biological Events is initiating pilot projects to examine data sources, integration, and predictive analysis. The recent increase in dengue cases internationally prompted the Office of Operations, Security and Preparedness, and the Subject Matter Expertise Center for Biological Events to establish collaborations, and investigate factors influencing dengue disease patterns in VHA facilities.

The National Weather Service has the mission to provide weather, water and climate data, forecasts and warnings for the protection of life and property, and enhancement of the national economy. The Veterans Affairs Integrated Operations Center enabled collaboration with the National Weather Service for integration of weather, water and climate data, and retrospective analysis into preparedness activities.

\section{Methods}

The VHA patient treatment file administrative dataset was queried for occurrence of dengue, and dengue-like diagnosis codes in VHA inpatients across the nation for Federal Fiscal Years (FYs) 2008, 2009, and 2010 (up to July).

The National Weather Service used Advanced Hydrologic Prediction Service precipitation data. This quality-controlled data is projected into gridded images to provide spatial information. Analysis fields included departures from average, and percent of average (daily, monthly, and for a water year). Spatial variation data were contrasted with the rain gauge point data available from National Oceanic and Atmospheric Administration's National Climatic Data Center archive.

The environmental data for FYs 2008-2010 were added to the VHA dengue case data to evaluate if weather and water influences can be associated with disease occurrence in a

This is an Open Access article distributed under the terms of the Creative Commons Attribution licence (http://creativecommons.org/licenses/by/2.5) open - access which permits unrestricted use, distribution, and reproduction in any medium, provided the original work is properly cited. 
patient population, with the intent of laying the building blocks for a predictive model of disease.

\section{Results}

As expected, dengue or dengue-like cases in VHA inpatients primarily occurred in Puerto Rico, where the disease is endemic. Thus, far in FY 2010, 35 cases have been recorded; FYs 2008 and 2009 had 31 and 14 cases, respectively. Interestingly, the case occurrence pattern was different for FY 2010 compared with FYs 2008 and 2009: cases in FY 2010 occurred in peaks in the winter, spring, and summer, whereas occurrence in the other years peaked only in the fall with low case numbers the rest of the year. The Advanced Hydrologic Prediction Service precipitation web portal provided access to images of observed precipitation, period normal, and departure from normal and percent of normal precipitation pre-compiled for time periods from one day to the water-year to date, to guide analysis of dengue fever outbreaks.

\section{Conclusions}

This work demonstrates the value and potential of Integrated Operations Center initiatives within VA (for example, Subject Matter Expertise Center for Biological Events), and external collaborations (for example, National Oceanic and Atmospheric Administration) toward integrated disease threat prediction, prevention, and mitigation capabilities. The outcomes will drive policy to enhance care of VA's Veteran patients, and support preparedness activities that will improve national decision-making in support of the National Response Framework.

\section{Acknowledgements}

This paper was presented as a poster at the 2010 International Society for Disease Surveillance Conference, held in Park City, UT, USA on 1-2 December 2010. 


\title{
Syndromic surveillance of influenza-like illness using automated VA data preliminary results from the Idaho infectious disease reporting network
}

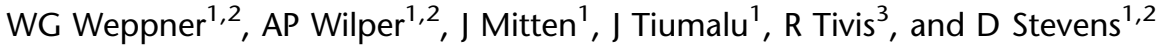 \\ ${ }^{1}$ Boise VA Medical Center, Boise, ID, USA; ${ }^{2}$ University of Washington School of Medicine, Seattle, WA, USA; and ${ }^{3}$ Idaho State University, \\ Pocatello, ID, USA \\ E-mail: William.Weppner@va.gov www.IIDRN.org
}

\begin{abstract}
Objective
The objective of this paper is to study whether syndromic surveillance using data from the Veterans Administration electronic medical record computerized patient record system (CPRS) correlates to officially reported influenza activity levels in the State of Idaho.
\end{abstract}

\section{Introduction}

Current influenza-like illness monitoring in Idaho is on the basis of syndromic surveillance using laboratory data, combined with periodic person-to-person reports collected by Idaho state workers. This system relies on voluntary reporting.

Electronic medical records with relational databases offer a method of obtaining data in an automated fashion. Clinical data entered in CPRS includes real-time visit information, vital signs, ICD-9, pharmacy, and labs. ICD-9 and vital signs have been used to predict influenza-like illness in automated systems. $^{1,2}$ We sought to combine these with lab and pharmacy data as part of an automated syndromic surveillance system.

\section{Methods}

The Boise Veterans Affairs Medical Center provides care to over 20,000 veterans living in Idaho, with clinics in Boise and surrounding sites. Using data from the Veterans Integrated Service Network (VISN 20) data warehouse for influenza from 2009, we identified influenza-like illness cases from these clinics using ICD-9 codes collected as weekly counts. Additional counts of fever $\left(>100.5^{\circ} \mathrm{F}\right)$, hypoxia $\left(\mathrm{O}_{2}<92 \%\right)$, lab tests for influenza (A/B antigen, culture, novel flu), and prescriptions for antivirals (oseltamivir) were summed individually, and in an unweighted fashion, as total weekly counts. Spearman correlation, and multivariate logistic regression were used with predictors from the same week, and preceding week. This was correlated with weekly flu activity as reported by the Center for Disease Control and Prevention; this reports geographic spread of influenza, as reported by state epidemiologists. $^{3}$ The Veterans Administration Puget Sound Institutional Review Board (IRB) approved this study.

\section{Results}

Using comparisons with epidemiologist-reported flu activity level, all clinical data elements had statistically significant associations using Spearman correlation: sum of total counts of predictors $r=0.57(P<0.0001)$; lab tests $r=0.51(0.0001)$; ICD-9 codes $r=0.47 \quad(P=0.0003) ;$ prescription $r=0.38$ $(P=0.005) ; \quad$ fever $r=0.31 \quad(P=0.02) ; \quad$ hypoxia $r=0.28$ $(P=0.04)$. Total counts accounted for one-third $\left(r^{2}=0.3\right)$ of variance. Similar results were found for preceding week counts. In logistic regression, both ICD-9, and lab counts were significant predictors (Wald $\chi^{2}=6.80, P=0.009$ and Wald $\chi^{2}=7.15, P=0.007$, respectively) (Figure 1 ).

\section{Limitations}

We sampled a small percent of the overall state population using Veterans Administration data; young people, women, and children are underrepresented.

\section{Conclusions}

Data obtained from electronic health records may be useful in predicting influenza-like illness on a regional basis. The combination of ICD-9 codes, vital signs, lab, and pharmacy data provided the best correlation with influenza. ICD-9 and lab counts both contribute independently to prediction, and should be considered to build a stronger model of prediction in our data.

\section{Acknowledgements}

This paper was presented as a poster at the 2010 International Society for Disease Surveillance Conference, held in Park City, UT, USA on 1-2 December 2010.

This is an Open Access article distributed under the terms of the Creative Commons Attribution licence (http://creativecommons.org/licenses/by/2.5) OPEN - ACCESS which permits unrestricted use, distribution, and reproduction in any medium, provided the original work is properly cited. 


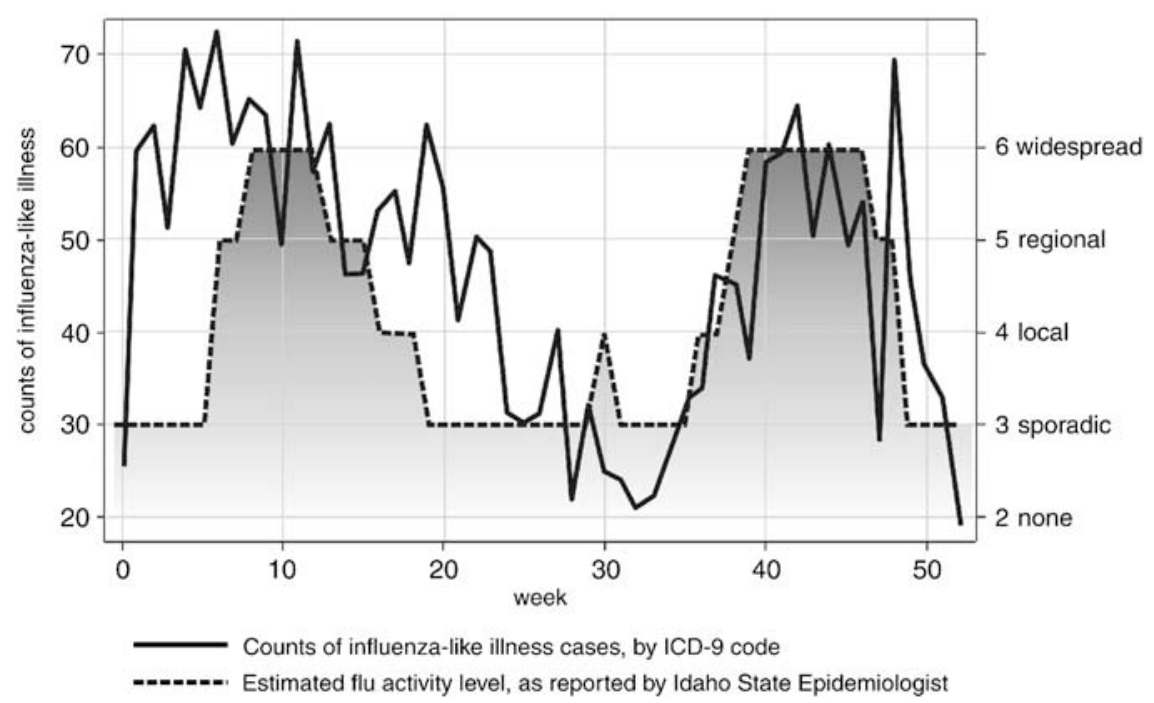

Figure 1 Veterans Administration influenza-like illness cases versus reported weekly influenza activity in the state of Idaho, 2009.

\section{References}

1 Marsden-Haug N, Foster VB, Gould PL, Elbert E, Wang H, Pavlin JA. Code-based syndromic surveillance for influenzalike illness by International Classification of Diseases, ninth revision. Emerg Infect Dis 2007;13:207-16.
2 Yih WK, Deshpande S, Fuller C, Heisey-Grove D, Hsu J, Kruskal BA, et al. Evaluating real-time syndromic surveillance signals from Ambulatory Care Data in four states. Public Health Rep 2010;125:111-25.

3 CDC. Fluview. Weekly US Map: Influenza Summary Update. http://www.cdc.gov/flu/weekly/usmap.htm. 


\title{
Evaluating University syndromic surveillance systems during the 2009 H1N1 influenza pandemic
}

\author{
Y Zhang ${ }^{1,2}$, L May ${ }^{1,2}$, and MA Stoto ${ }^{1,2}$ \\ ${ }^{1}$ Georgetown University, Washington, DC, USA; and ${ }^{2}$ George Washington University, Washington, DC, USA \\ E-mail: yz62@georgetown.edu
}

\section{Objective}

To describe the 2009 H1N1 outbreak at Georgetown University (GU) and George Washington University (GWU) in Fall 2009. Identify the datasets that most accurately depict 2009 H1N1 disease in real time.

\section{Introduction}

Syndromic surveillance has been widely adopted as a realtime monitoring tool in early response to disease outbreaks. In order to provide real-time information on the impact of 2009 H1N1 during the Fall 2009 semester, GU and GWU employed syndromic surveillance systems incorporating a variety of data sources.

\section{Methods}

The data series include:

- Student influenza-like illness (ILI) cases reported to medical staff: ED visits (GU and GWU Hospital emergency room visits records for patients aged 17-24 years with ILI),
SHC (total of visits to the GU and GWU student health centers), off-hour calls to the GU student health center, and calls to the nurse-operated GU H1N1 advice line.

- GU student absenteeism: ILI cases reported to deans at four undergraduate colleges, athletic trainers, and resident assistants.

- GU employee absenteeism data: Real-time employee absenteeism based on call-in sick log at the Facilities Office and Dining Services, and retrospective employee absenteeism in 2008 and 2009 based on payroll data.

- External surveillance data: American College Health Association Pandemic Influenza Surveillance Network, and the CDC ILINet.

\section{Results}

- ILI cases reported to medical staff, especially ED visits, peaked first in early September and dropped sharply afterwards. This is similar at GU and GWU, and corresponds to regional and national ACHA data, but

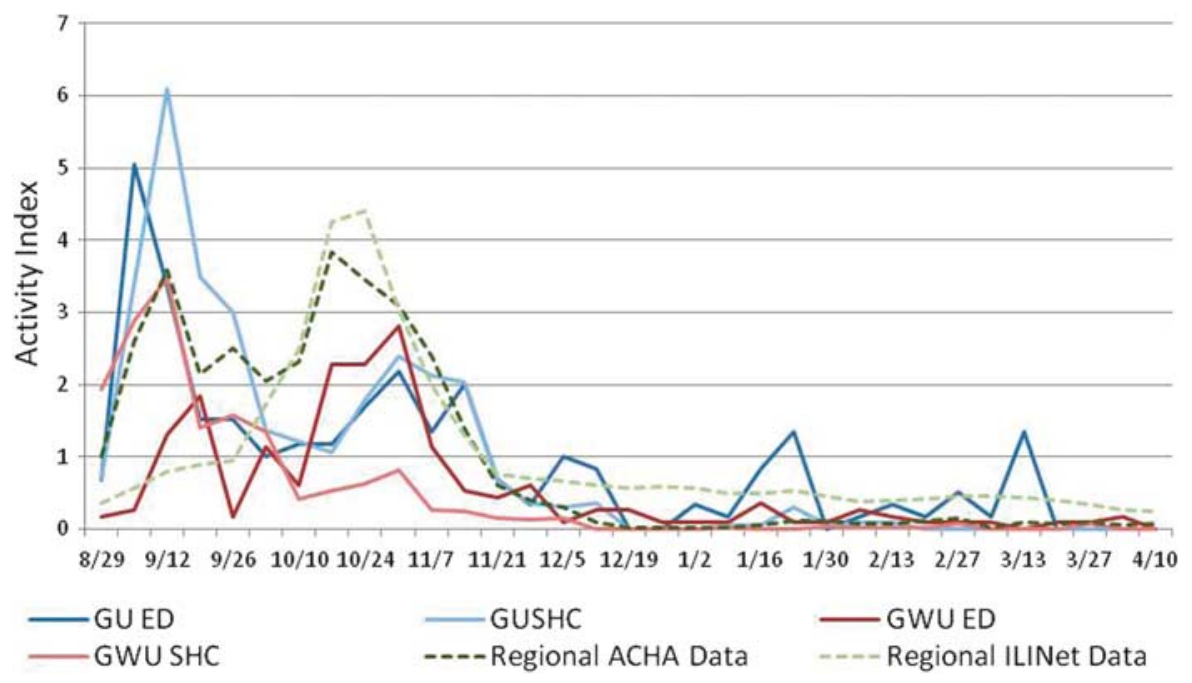

Figure $1 \mathrm{GU}$ and GWU ILI cases.

\footnotetext{
This is an Open Access article distributed under the terms of the Creative Commons Attribution licence (http://creativecommons.org/licenses/by/2.5) OPEN - ACCESS which permits unrestricted use, distribution, and reproduction in any medium, provided the original work is properly cited.
} 
not Region 3 (DE, DC, MD, PA, VA, WV) ILINet data (Figure 1).

- Student self-reports collected by deans exhibited a second peak in weeks around midterms.

- Retrospective employee absenteeism patterns are similar to those of the previous year (Figure 2).

\section{Discussion}

- 2009 H1N1 activity at both GU and GWU seems to peak right after school begins, which can be observed in a variety of data sources. The second peak in late October corresponds to regional ILINet data but does not show the same scope of impact on campus. This might reflect the epidemic in the community and the increasing need for student medical notes during midterm weeks (Figure 3).

- Syndromic surveillance data can be strongly influenced by factors that influence people's reporting behavior such as communication and intervention policies, and incentives and barriers associated with presenting oneself to the reporting system.

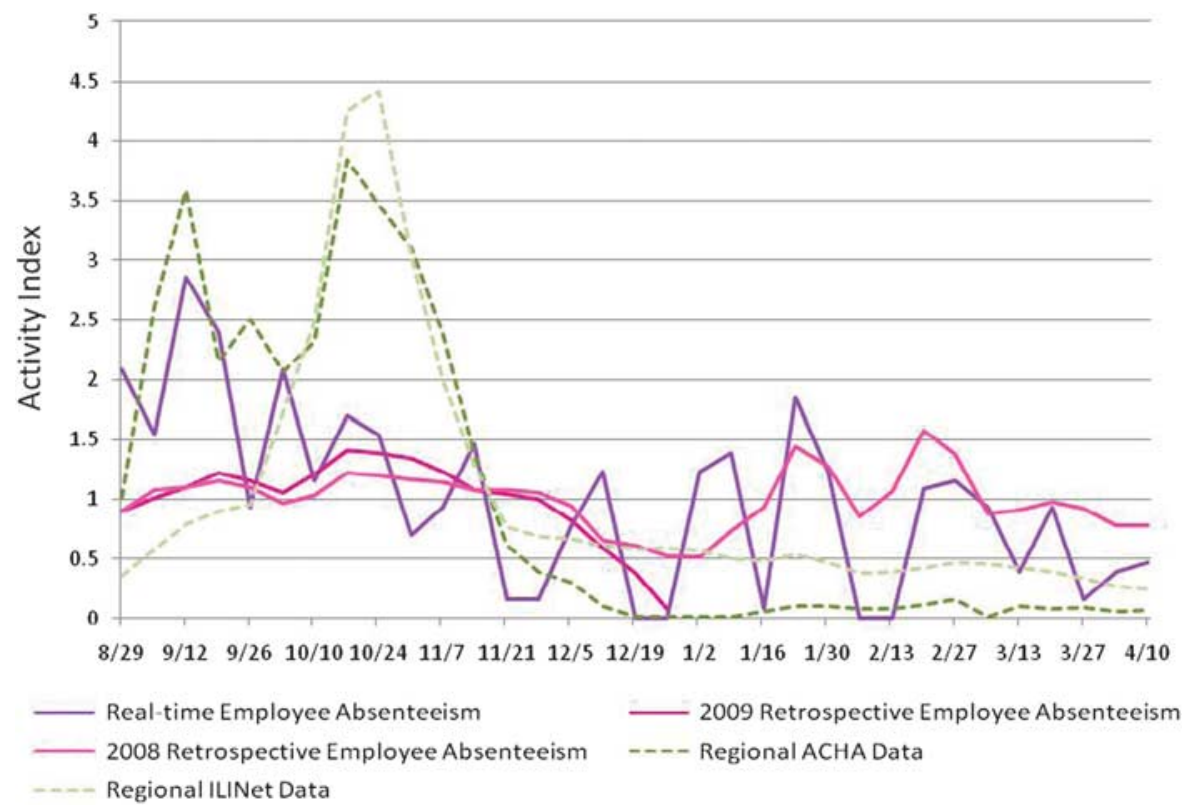

Figure $2 \mathrm{GU}$ employee absenteeism data.

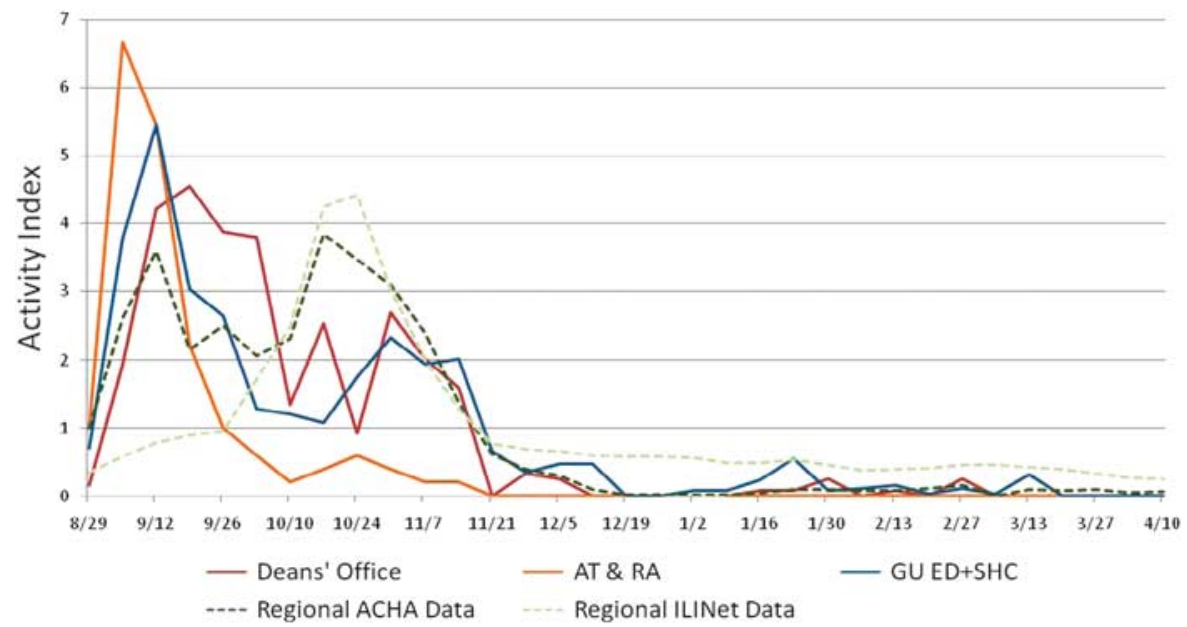

Figure $3 \mathrm{GU}$ student absenteeism data. 
- The SHC data may be the most reliable data source in the early stages of an outbreak, but completeness of reporting seems to fall off with policy changes and as students may subsequently see no value to seeking medical attention.

\section{Acknowledgements}

This paper was presented as a poster at the 2010 International Society for Disease Surveillance Conference, held in Park City, UT, USA, on 1-2 December 2010. 